\title{
SPECTRAL STATISTICS FOR RANDOM SCHRÖDINGER OPERATORS IN THE LOCALIZED REGIME
}

\author{
FRANÇOIS GERMINET AND FRÉDÉRIC KLOPP
}

\begin{abstract}
We study various statistics related to the eigenvalues and eigenfunctions of random Hamiltonians in the localized regime. Consider a random Hamiltonian at an energy $E$ in the localized phase. Assume the density of states function is not too flat near $E$. Restrict it to some large cube $\Lambda$. Consider now $I_{\Lambda}$, a small energy interval centered at $E$ that asymptotically contains infintely many eigenvalues when the volume of the cube $\Lambda$ grows to infinity. We prove that, with probability one in the large volume limit, the eigenvalues of the random Hamiltonian restricted to the cube inside the interval are given by independent identically distributed random variables, up to an error of size an arbitrary power of the volume of the cube.

As a consequence, we derive

- uniform Poisson behavior of the locally unfolded eigenvalues,

- a.s. Poisson behavior of the joint distibutions of the unfolded energies and unfolded localization centers in a large range of scales.

- the distribution of the unfolded level spacings, locally and globally,

- the distribution of the unfolded localization centers, locally and globally.
\end{abstract}

RÉSUMÉ. Nous étudions différentes statistiques associées aux valeurs propres et vecteurs propres d'un opérateur aléatoire dans le régime localisé. Considérons un opérateur aléatoire au voisinage d'une énergie $E$ supposée se trouver dans le régime localisé. On considère la restirction de l'opérateur à un grand cube $\Lambda$. Soit $I_{\Lambda}$, un petit intervalle d'énergie centré en $E$ qui, asymptotiquement, contient une infinité de valeurs propres quand $\Lambda$ grandit. Nous démontrons qu'asymptotiquement presque sûrement, les valeurs propres de l'opérateur aléatoire restreint au cube contenu dans l'intervalle sont très bien approchées par des variables aléatoires indépendantes identiquement distribuées.

De cette caractérisation, nous déduisons

- que localement, les valeurs propres renormalisées comportement uniformément poissonien,

- que les distibutions jointes des valeurs propres et centres de localisation renormalisés sont asymptotiquement distribuées selon une loi de Poisson,

- la distribution des espacements de niveaux renormalisés, localement et globalement,

- la distribution des espacements des centres de localisation renormalisés, localement et globalement.

\section{InTRODUCTION}

To introduce our results, we first restrict our exposition to the celebrated the random Anderson model, that is, on $\ell^{2}\left(\mathbb{Z}^{d}\right)$, we consider the operator

$$
H_{\omega}=-\Delta+V_{\omega}
$$

2000 Mathematics Subject Classification. 81Q10,47B80,60H25,82D30,35P20.

Key words and phrases. random Schrödinger operators, eigenvalue statistics, level spacing distribution.

The authors are supported by the grant ANR-08-BLAN-0261-01. The authors would also like to thank the Banff International Research Station (Alberta, Canada) and the Centre Interfacultaire Bernoulli (EPFL, Lausanne), where this work was partially written, for their hospitality.

It is pleasure to thank C. Shirley and N. veniaminov for pointing out various mistakes in previous versions of the paper. 
where $-\Delta$ is the free discrete Laplace operator

$$
(-\Delta u)_{n}=\sum_{|m-n|=1} u_{m} \quad \text { for } u=\left(u_{n}\right)_{n \in \mathbb{Z}^{d}} \in \ell^{2}\left(\mathbb{Z}^{d}\right)
$$

and $V_{\omega}$ is the random potential

$$
\left(V_{\omega} u\right)_{n}=\omega_{n} u_{n} \quad \text { for } u=\left(u_{n}\right)_{n \in \mathbb{Z}^{d}} \in \ell^{2}\left(\mathbb{Z}^{d}\right) .
$$

We assume that the random variables $\left(\omega_{n}\right)_{n \in \mathbb{Z}^{d}}$ are independent identically distributed and that their distribution admits a compactly supported smooth density, say $g$.

It is well known (see e.g. [Kir08]) that, $\omega$ almost surely, the spectrum of $H_{\omega}$ is equal to a fixed closed set, say, $\Sigma$. Moreover, there exists a Lebesgue almost everywhere bounded density of states, say $\lambda \mapsto \nu(\lambda)$, such that, for any continuous function $\varphi: \mathbb{R} \rightarrow \mathbb{R}$, one has

$$
\int_{\mathbb{R}} \varphi(\lambda) \nu(\lambda) d \lambda=\mathbb{E}\left(\left\langle\delta_{0}, \varphi\left(H_{\omega}\right) \delta_{0}\right\rangle\right) .
$$

The function $\nu$ is the density of a probability measure on $\Sigma$.

For $L>1$, consider $\Lambda=[-L, L]^{d} \cap \mathbb{Z}^{d}$, a cube on the lattice and let $H_{\omega}(\Lambda)$ be the random Hamiltonian $H_{\omega}$ restricted to $\Lambda$ with periodic boundary conditions. It is a finite dimensional symmetric matrix; let us denote its eigenvalues ordered increasingly and repeated according to multiplicity by $E_{1}(\omega, \Lambda) \leq E_{2}(\omega, \Lambda) \leq \cdots \leq E_{N}(\omega, \Lambda)$. For $x \geq 0$, define the empirical level spacings distribution of $H_{\omega}(\Lambda)$ as

$$
D L S(x ; \omega, \Lambda)=\frac{\#\left\{j ;\left(E_{j+1}(\omega, \Lambda)-E_{j}(\omega, \Lambda)\right)|\Lambda| \geq x\right\}}{|\Lambda|} .
$$

A result that is typical of the results we prove in the present paper is

Theorem 0.1. There exists $\lambda_{0}>0$ such that, for $|\lambda|>\lambda_{0}$, with probability 1, as $|\Lambda| \rightarrow+\infty$, $D L S(x ; \omega, \Lambda)$ converges uniformly to the distribution $x \mapsto g(x)$ where

$$
g(x)=\int_{\Sigma} \mathrm{e}^{-\nu(\lambda) x} \nu(\lambda) d \lambda
$$

that is, $\omega$ almost surely,

$$
\sup _{x \geq 0}|D L S(x ; \omega, \Lambda)-g(x)| \underset{|\Lambda| \rightarrow+\infty}{\rightarrow} 0 .
$$

This result shows that, for the discrete Anderson Hamiltonian with smoothly distributed random potential at sufficiently large coupling, the limit of the level spacings distribution is that of i.i.d. random variables distributed according to the density of states of the random Hamiltonian.

To the best of our knowledge, this is the first rigorous study of the level spacings distribution of random Schrödinger operators.

The purpose of the paper is to study spectral statistics for random Hamiltonians in the localized regime. The large coupling Anderson Hamiltonian described above is the typical example. Spectral statistics have been studied in various works, mainly for discrete or continuous Anderson models (see e.g. [Mol82, Min96, KN07, Wan01]) but up to now, to the best of our knowledge, studies have only described the local spectral statistics. For a random Hamiltonian restricted to a cube $\Lambda$, the existence of the density of states, that is, of a limit for the number of eigenlevels per unit of volume (see (1.3)) implies that the average distance between levels is of order $|\Lambda|^{-1}$. Local spectral statistics are the statistics in energy intervals $I$ of the size $|\Lambda|^{-1}$. Thus, such intervals contain typically a number of eigenvalues that is bounded uniformly in the volume of the cube. This, in particular, prevents the study of the empirical distribution of level spacings.

In the present paper, we go beyond this. Therefore, we introduce a new method of study of the 
eigenlevels and localization centers that is quite close to the physical heuristics (see e.g. [Jan98, LR85, Mir00]). The method consists in approximating the eigenvalues of the true random operator (restricted to some finite cube) by a family of i.i.d. random variables that are constructed as eigenvalues of the random operator restricted to smaller cubes. That this is possible is a consequence of localization: due to their exponential falloff, eigenfunctions only see the random potential surrounding them. This construction is only feasible under some restrictions on the relative size of the region where one wants to study eigenvalues and the size of the cube on which one restricts the random operator. If one wants to control, with a good probability, all the eigenvalues in some interval $I$, then, one roughly needs $I$ to be of size $|\Lambda|^{-\alpha}$, the inverse of the volume of the cube to some power $\alpha$ smaller than but close to 1 (see Theorem 1.1). If one wants to enlarge the interval $I$, one can go up to sizes that are of order a negative power of $\log |\Lambda|$ at the expense of being able to describe only most of the eigenvalues (see Theorem 1.2).

The basic tools that we use to control the eigenvalues are the so-called "Wegner" and "Minami" estimates (see (W) and (M) in section 1.1).

Using the approximation described above, we obtain a large deviation estimate for the number of eigenvalues inside a possibly shrinking interval of a random operator restricted to some large cube (see Theorem 1.3). This bound shows that, for intervals $I$ that are not too small, with good probability, the number of eigenvalues in $I$ is given by the weight that the integrated density of states gives to $I$ times the volume of the cube up to an error of smaller order.

Then, we derive the almost sure level spacings statistics near fixed energies as well as inside non trivial compact intervals (see Theorems 1.4, 1.6 and 1.7). We also compute the localization center spacings statistics (see Theorem 1.7).

This is the first time that these statistics are obtained for random Schrödinger operators.

The next result is the uniform local statistic for the eigenvalues and localization centers when they are rescaled covariantly i.e. the scaling in energy is of order the scaling in space to the power $-d$ (see Theorem 1.10); we prove that the covariantly scaled local statistics are independent of the scale (if they are not too small) i.e. one always obtains Poisson statistics. In the non-covariant scaling case, we obtain almost sure results on the counting function (see Theorem 1.15). In the case of the standard scale i.e. energies are scaled by $|\Lambda|^{-1}$ on a cube of volume $\Lambda$, we also study the asymptotic independence of these local processes (see Theorems 1.11 and 1.12). This extends known results of [Mol82, Min96, KN07].

We point out that our analysis goes beyond the previous results also in the sense that locally the images of the eigenvalues by the IDS are shown to exhibit a Poissonian behavior. When the DOS exists and is non zero, we recover the previous known statements, but a vanishing derivative of the IDS is allowed in the present work.

We also consider the problem from a different point of view. The usual procedure consists in restricting the random Hamiltonian to some finite cube and study the statistics for this operator in the limit when the cube grows to be the whole space. One can also consider the Hamiltonian in the whole space. In a compact interval in the localized region, say $I$, the Hamiltonian admits countably many eigenvalues. We enumerate them using the localization center attached to an associated eigenfunction (see Proposition 1.2). I.e. we consider the eigenvalues in $I$ having localization in some finite cube. We derive the almost sure statistics of the level spacings distributions (see Theorem 1.8); they are the same as the ones obtained for the Hamiltonian restricted to a cube.

Finally let us us conclude this introduction by saying that a number of the results obtained in the present paper for general random Schrödinger operators were already described for discrete random operators in [GKl11]. 


\section{The MAIN Results}

After this short illustration of what can be obtained from our method, we now turn to the description of the main results of this paper. Results will be given for general random Schrödinger operators under a number of assumptions that are known to hold for, e.g. the Anderson model and some continuous Anderson models.

We shall use the following standard notations: $a \lesssim b$ means there exists $c<\infty$ so that $a \leq c b$; $a \asymp b$ means $a \lesssim b$ and $b \lesssim a ;\langle x\rangle=\left(1+|x|^{2}\right)^{\frac{1}{2}}$.

1.1. The random model. Consider $H_{\omega}=H_{0}+V_{\omega}$, a $\mathbb{Z}^{d}$-ergodic random Schrödinger operator on $\mathcal{H}=L^{2}\left(\mathbb{R}^{d}\right)$ or $\ell^{2}\left(\mathbb{Z}^{d}\right)$ (see e.g. [PF92, Sto01]). Typically, the background potential $H_{0}$ is the Laplacian $-\Delta$, possibly perturbed by a periodic potential. Magnetic fields can be considered as well; in particular, the Landau Hamiltonian is also admissible as a background Hamiltonian. For the sake of simplicity, we assume that $V_{\omega}$ is almost surely bounded; hence, almost surely, $H_{\omega}$ share the same domain $H^{2}\left(\mathbb{R}^{d}\right)$ or $\ell^{2}\left(\mathbb{Z}^{d}\right)$.

For $\Lambda$, a cube in either $\mathbb{R}^{d}$ or $\mathbb{Z}^{d}$, we let $H_{\omega}(\Lambda)$ be the operator $H_{\omega}$ restricted to $\Lambda$ with periodic boundary conditions. Our analysis stays valid for Dirichlet boundary conditions.

Furthermore, we shall denote by $\mathbf{1}_{J}(H)$ the spectral projector of the operator $H$ on the energy interval $J . \mathbb{E}(\cdot)$ denotes the expectation with respect to $\omega$; Our first assumption will be an independence assumption on the local Hamiltonian that is

(IAD): Independence At a Distance: there exists $R_{0}>0$ such that for $\operatorname{dist}\left(\Lambda, \Lambda^{\prime}\right)>R_{0}$, the random Hamiltonians $H_{\omega}(\Lambda)$ and $H_{\omega}\left(\Lambda^{\prime}\right)$ are independent.

Such an assumption is clearly satisfied by standard models like the Anderson model, the Poisson model or the random displacement model if the single site potential is compactly supported (see e.g. [PF92, Sto01]).

Remark 1.1. As will be seen in the course of the proofs, it can be weakened to assuming that the correlations between the local Hamiltonians decay polynomially at a rate that is faster than the $-d$-th power of the distance separating the cubes on which the local Hamiltonians are considered.

Let $\Sigma$ be the almost sure spectrum of $H_{\omega}$. Pick $I$ a relatively compact open subset of $\Sigma$. Assume the following holds:

(W): a Wegner estimate holds in $I$, i.e. given $I$ there exists $C>0$ such that, for $J \subset I$, and $\Lambda$, a cube in $\mathbb{R}^{d}$ or $\mathbb{Z}^{d}$, one has

$$
\mathbb{E}\left[\operatorname{tr}\left(\mathbf{1}_{J}\left(H_{\omega}(\Lambda)\right)\right)\right] \leq C|J||\Lambda| .
$$

(M): a Minami estimate holds in $I$, i.e. given $I$ there exists $C>0$ and $\rho>0$ such that, for $J \subset I$, and $\Lambda$, a cube in $\mathbb{R}^{d}$ or $\mathbb{Z}^{d}$, one has

$$
\mathbb{E}\left[\operatorname{tr}\left(\mathbf{1}_{J}\left(H_{\omega}(\Lambda)\right)\right) \cdot\left[\operatorname{tr}\left(\mathbf{1}_{J}\left(H_{\omega}(\Lambda)\right)\right)-1\right]\right] \leq C(|J||\Lambda|)^{1+\rho} .
$$

Remark 1.2. The Wegner estimate has been proved for many random Schrödinger models both discrete and continuous Anderson models under rather general conditions on the single site potential and on the randomness (see e.g. [His08, KM07, Ves08]). The right hand side in (1.1) can be lower bounded by the probability to have at least one eigenvalue in $J$ (for $J$ small).

As the proofs will show, one can weaken assumption (W) and replace the right hand side with $C|J|^{\alpha}|\Lambda|^{\beta}$ for arbitrary positive $\alpha$ and $\beta$. Such Wegner estimates are known to hold also for some non monotonous models (see e.g. [K195, HK02, GHK07]).

On the Minami estimate, much less is known: it holds for the discrete Anderson model with $I=\Sigma$ (see [Min96, GV07, BHS07, CGK09]). These proofs yield an optimal exponent $\rho=1$. 
In dimension 1, it has been proved recently (see [Kl12]), that, for general random models, the Minami estimate (for any $\rho \in(0,1)$ ) is a consequence of the Wegner estimate within the localization region (see section 1.2).

In higher dimensions, for continuous Anderson models, proving a Minami estimate is still a challenging open problem. The right hand side in (1.2) can be lower bounded by the probability to have at least two eigenvalues in $J$. For $\rho=1$, it behaves as the square of the probability to have one eigenvalue in $J$. So, roughly speaking, close by eigenvalues behave as independent random variables.

The integrated density of states is defined as

$$
N(E):=\lim _{|\Lambda| \rightarrow+\infty} \frac{\#\left\{\text { e.v. of } H_{\omega}(\Lambda) \text { less than } \mathrm{E}\right\}}{|\Lambda|} .
$$

By $(\mathrm{W}), N(E)$ is the distribution function of a measure that is absolutely continuous with respect to the Lebesgue measure on $\mathbb{R}$. Let $\nu$ be the density of state of $H_{\omega}$ i.e. the distributional derivative of $N$. In the sequel, for a set $I$, we will often write $N(I)$ for the mass the measure $\nu(E) d E$ puts on $I$ i.e.

$$
N(I)=\int_{I} \nu(E) d E .
$$

1.2. The localized regime. For $L \geq 1, \Lambda_{L}$ denotes the cube $[-L / 2, L / 2]^{d}$ in either $\mathbb{R}^{d}$ or $\mathbb{Z}^{d}$. Let $\mathcal{H}_{\Lambda}$ be $\ell^{2}\left(\Lambda \cap \mathbb{Z}^{d}\right)$ in the discrete case and $L^{2}(\Lambda)$ in the continuous one. For a vector $\varphi \in \mathcal{H}$, we define

$$
\|\varphi\|_{x}=\left\{\begin{array}{cl}
\left\|\mathbf{1}_{\Lambda(x)} \varphi\right\|_{2} \text { where } \Lambda(x)=\{y ;|y-x| \leq 1 / 2\} & \text { if } \mathcal{H}=L^{2}\left(\mathbb{R}^{d}\right), \\
|\varphi(x)| & \text { if } \mathcal{H}=\ell^{2}\left(\mathbb{Z}^{d}\right) .
\end{array}\right.
$$

In the discrete case, the definition is that given in section 1.8.

Let $I$ be a compact interval. We assume that $I$ lies in the region of complete localization (see e.g. [GK04, GK06]) for which we use the following finite volume version:

(Loc): for all $\xi \in(0,1)$, one has

$$
\sup _{L>0} \sup _{\substack{\text { supp } f \subset I \\|f| \leq 1}} \mathbb{E}\left(\sum_{\gamma \in \mathbb{Z}^{d}} \mathrm{e}^{|\gamma|^{\xi}}\left\|\mathbf{1}_{\Lambda(0)} f\left(H_{\omega}\left(\Lambda_{L}\right)\right) \mathbf{1}_{\Lambda(\gamma)}\right\|_{2}\right)<+\infty .
$$

Whenever the fractional moment method is available, one may replace the factor $\mathrm{e}^{|\gamma|^{\xi}}$ by an exponential one $\mathrm{e}^{\eta|\gamma|}$, where $\eta>0$.

Remark 1.3. Assumption (Loc) may be relaxed into asking (1.6) for a single $\xi$. This will not change the subsequent results in an essential way, but only modify some constants.

We note that the assumption (Loc) implies in particular that the spectrum of $H_{\omega}$ is pure point in $I$ (see e.g. [GK06, Kir08]). We refer to Appendix 6.2 where, in Theorem 6.1, we provide equivalent finite volume properties of the region of complete localization, and show it coincides with the infinite volume one. For the sake of the exposition, from Theorem 6.1, we extract the following lemma that we shall use intensively in this paper.

Lemma 1.1. Assume $(W)$ and (Loc).

(I) For any $p>0$ and $\xi \in(0,1)$, for $L \geq 1$ large enough, there exists a set of configuration $\mathcal{U}_{\Lambda_{L}}$ such that $\mathbb{P}\left(\mathcal{U}_{\Lambda_{L}}\right) \geq 1-L^{-p}$ and for $\omega \in \mathcal{U}_{\Lambda_{L}}$, if

(1) $\varphi_{j}\left(\omega, \Lambda_{L}\right)$ is a normalized eigenvector of $H_{\omega}\left(\Lambda_{L}\right)$ associated to $E_{j}\left(\omega, \Lambda_{L}\right) \in I$,

(2) $x_{j}\left(\omega, \Lambda_{L}\right) \in \Lambda_{L}$ is a maximum of $x \mapsto\left\|\varphi_{j}\left(\omega, \Lambda_{L}\right)\right\|_{x}$ in $\Lambda_{L}$, 
then, for $x \in \Lambda_{L}$, one has

$$
\left\|\varphi_{j}\left(\omega, \Lambda_{L}\right)\right\|_{x} \leq L^{p+d} \mathrm{e}^{-\left|x-x_{j}\left(\omega, \Lambda_{L}\right)\right|^{\xi}}
$$

(II) For any $\nu, \xi \in(0,1), \nu<\xi$, for $L \geq 1$ large enough, there exists a set of configuration $\mathcal{V}_{\Lambda_{L}}$ such that $\mathbb{P}\left(\mathcal{V}_{\Lambda_{L}}\right) \geq 1-e^{-L^{\nu}}$, and for $\omega \in \mathcal{V}_{\Lambda_{L}}$, if

(1) $\varphi_{j}\left(\omega, \Lambda_{L}\right)$ is a normalized eigenvector of $H_{\omega}\left(\Lambda_{L}\right)$ associated to $E_{j}\left(\omega, \Lambda_{L}\right) \in I$,

(2) $x_{j}\left(\omega, \Lambda_{L}\right) \in \Lambda_{L}$ is a maximum of $x \mapsto\left\|\varphi_{j}\left(\omega, \Lambda_{L}\right)\right\|_{x}$ in $\Lambda_{L}$,

then, for $x \in \Lambda_{L}$, one has

$$
\left\|\varphi_{j}\left(\omega, \Lambda_{L}\right)\right\|_{x} \leq e^{2 L^{\nu}} e^{-\left|x-x_{j}\left(\omega, \Lambda_{L}\right)\right|^{\xi}} .
$$

Remark 1.4. Both Part (I) and (II) of Lemma 1.1 are consequences of the localization hypothesis. We shall use both of these characterizations of localization. Part (I) is relevant for large scales (typically, powers of the volume of the reference box) and yields a smaller constant in front of the exponential, while part (II) will be used for smaller scales (typically powers of log of the volume of the box) and yields a better probability.

Such a result can essentially be found in [GK06] for the continuous case and in [K111] for the discrete case.

Clearly, the function $x \mapsto\left\|\varphi_{j}\left(\omega, \Lambda_{L}\right)\right\|_{x}$ need not have a unique maximum in $\Lambda_{L}$. But, as, for any $x \in \Lambda_{L}$, one has

$$
\sum_{\gamma \in \Lambda_{L} \cap \mathbb{Z}^{d}}\left\|\varphi_{j}\left(\omega, \Lambda_{L}\right)\right\|_{x+\gamma}^{2}=\left\|\varphi_{j}\left(\omega, \Lambda_{L}\right)\right\|^{2}=1,
$$

if $x_{j}\left(\omega, \Lambda_{L}\right)$ is a maximum, then $\left\|\varphi_{j}\left(\omega, \Lambda_{L}\right)\right\|_{x_{n}(\omega)}^{2} \geq(2 L+1)^{-d}$. Hence, if $x_{j}\left(\omega, \Lambda_{L}\right)$ and $x_{j}^{\prime}\left(\omega, \Lambda_{L}\right)$ are two maxima, then (Loc), through Lemma 1.1(I), implies that, for any $p$, there exists $C_{p}>0$ such that, with a probability larger than $1-L^{-p}$, we have

$$
\left|x_{j}\left(\omega, \Lambda_{L}\right)-x_{j}^{\prime}\left(\omega, \Lambda_{L}\right)\right| \leq C_{p}(\log L)^{1 / \xi}
$$

For $\varphi \in \mathcal{H}_{\Lambda}$, define the set of localization centers for $\varphi$ as

$$
C(\varphi)=\left\{x \in \Lambda ;\|\varphi\|_{x}=\max _{\gamma \in \Lambda}\|\varphi\|_{\gamma}\right\}
$$

As a consequence of Lemma 1.1, one has

Lemma 1.2. Pick $I$ in the localized regime for $H_{\omega}$. For any $p>0$, there exists $C_{p}>0$, such that, with probability larger than $1-L^{-p}$, if $E_{j}\left(\omega, \Lambda_{L}\right) \in I$ then the diameter of $C\left(\varphi_{j}\left(\omega, \Lambda_{L}\right)\right)$ is less than $C_{p} \log ^{1 / \xi}|\Lambda|$.

From now on, a localization center for a function $\varphi$ will denote any point in the set of localization centers $C(\varphi)$ and let $x_{j}\left(\omega, \Lambda_{L}\right)$ be a localization center for $\varphi_{j}\left(\omega, \Lambda_{L}\right)$. One can e.g. order them lexicographically and pick the one with largest coefficients.

1.3. The asymptotic description of the eigenvalues. We now state our main results. They are also the main technical results on which we base all our studies of the spectral statistics.

They consist in a precise approximation of the eigenvalues of $H_{\omega}(\Lambda)$ in $I_{\Lambda}$ by independent random variables, that follows in a rather straightforward way from the standard properties of random Schrödinger operators recalled above ((IAD), (W), (M) and localization). This approximation is at the heart of the proofs of the new statistical results we present in this paper: from it, we derive estimations on the number of eigenvalues in small intervals, we provide the first computation of the level spacings distribution, and we extend the known results about the convergence to Poisson of rescaled (or unfolded) eigenvalues.

We will give two different descriptions depending on the size of $N\left(I_{\Lambda}\right)$. When this quantity is sufficiently small with respect to $|\Lambda|^{-1}$, our procedure enables us to control all the eigenvalues. If 
it is not, we only control most of the eigenvalues.

Recall that our cube of reference is $\Lambda=\Lambda_{L}$, with center 0 and sidelength $L$.

1.3.1. Controlling all the eigenvalues. To start, pick $\tilde{\rho}$ such that

$$
0 \leq \tilde{\rho}<\frac{\rho}{1+d \rho}
$$

Assume $E_{0}$ is such that (1.42) holds. Now, pick $I_{\Lambda}$ centered at $E_{0}$ such that $N\left(I_{\Lambda}\right) \asymp|\Lambda|^{-\alpha}$ for $\alpha \in\left(\alpha_{d, \rho, \tilde{\rho}}, 1\right)$ where $\alpha_{d, \rho, \tilde{\rho}}$ is defined as

$$
\alpha_{d, \rho, \tilde{\rho}}:=(1+\tilde{\rho}) \frac{d \rho+1}{d \rho+1+\rho},
$$

where $\tilde{\rho}>0$ and $\rho$ is defined in the Minami estimate (M). Assumption (1.10) clearly implies that $\alpha_{d, \rho, \tilde{\rho}}<1$.

Our restriction will enable us to control all the eigenvalues of $H_{\omega}(\Lambda)$ in $I_{\Lambda}$.

Theorem 1.1. Assume $E_{0}$ is such that (1.42) holds for some $\tilde{\rho} \in[0, \rho /(1+d \rho))$. Recall that $\alpha_{d, \rho, \tilde{\rho}}$ is defined in (1.11) and pick $\alpha \in\left(\alpha_{d, \rho, \tilde{\rho}}, 1\right)$. Pick $I_{\Lambda}$ centered at $E_{0}$ such that $N\left(I_{\Lambda}\right) \asymp|\Lambda|^{-\alpha}$. There exists $\beta>0$ and $\beta^{\prime} \in(0, \beta)$ small so that $1+\beta \rho<\alpha \frac{1+\rho}{1+\tilde{\rho}}$ and, for $\ell \asymp L^{\beta}$ and $\ell^{\prime} \asymp L^{\beta^{\prime}}$, there exists a decomposition of $\Lambda$ into disjoint cubes of the form $\Lambda_{\ell}\left(\gamma_{j}\right):=\gamma_{j}+[0, \ell]^{d}$ satisfying:

- $\cup_{j} \Lambda_{\ell}\left(\gamma_{j}\right) \subset \Lambda$,

- $\operatorname{dist}\left(\Lambda_{\ell}\left(\gamma_{j}\right), \Lambda_{\ell}\left(\gamma_{k}\right)\right) \geq \ell^{\prime}$ if $j \neq k$,

- $\operatorname{dist}\left(\Lambda_{\ell}\left(\gamma_{j}\right), \partial \Lambda\right) \geq \ell^{\prime}$

- $\left|\Lambda \backslash \cup_{j} \Lambda_{\ell}\left(\gamma_{j}\right)\right| \lesssim|\Lambda| \ell^{\prime} / \ell$,

and such that, for $L$ sufficiently large, there exists a set of configurations $\mathcal{Z}_{\Lambda}$ s.t.:

- $\mathbb{P}\left(\mathcal{Z}_{\Lambda}\right) \geq 1-|\Lambda|^{-\left(\alpha-\alpha_{d, \rho, \tilde{\rho}}\right)}$,

- for $\omega \in \mathcal{Z}_{\Lambda}$, each centers of localization associated to $H_{\omega}(\Lambda)$ belong to some $\Lambda_{\ell}\left(\gamma_{j}\right)$ and each box $\Lambda_{\ell}\left(\gamma_{j}\right)$ satisfies:

(1) the Hamiltonian $H_{\omega}\left(\Lambda_{\ell}\left(\gamma_{j}\right)\right)$ has at most one eigenvalue in $I_{\Lambda}$, say, $E_{j}\left(\omega, \Lambda_{\ell}\left(\gamma_{j}\right)\right)$;

(2) $\Lambda_{\ell}\left(\gamma_{j}\right)$ contains at most one center of localization, say $x_{k_{j}}(\omega, \Lambda)$, of an eigenvalue of $H_{\omega}(\Lambda)$ in $I_{\Lambda}$, say $E_{k_{j}}(\omega, \Lambda)$;

(3) $\Lambda_{\ell}\left(\gamma_{j}\right)$ contains a center $x_{k_{j}}(\omega, \Lambda)$ if and only if $\sigma\left(H_{\omega}\left(\Lambda_{\ell}\left(\gamma_{j}\right)\right)\right) \cap I_{\Lambda} \neq \emptyset$; in which case, one has

$$
\left|E_{k_{j}}(\omega, \Lambda)-E_{j}\left(\omega, \Lambda_{\ell}\left(\gamma_{j}\right)\right)\right| \leq e^{-\left(\ell^{\prime}\right)^{\xi}} \text { and } \operatorname{dist}\left(x_{k_{j}}(\omega, \Lambda), \Lambda \backslash \Lambda_{\ell}\left(\gamma_{j}\right)\right) \geq \ell^{\prime}
$$

In particular if $\omega \in \mathcal{Z}_{\Lambda}$, all the eigenvalues of $H_{\omega}(\Lambda)$ are described by (1.12).

With a probability tending to 1 , Theorem 1.1 describes all the eigenvalues of $H_{\omega}(\Lambda)$ inside a sufficiently small interval $I_{\Lambda}$ as i.i.d. random variables defined as the unique eigenvalue of a copy of the random Hamiltonian $H_{\omega}\left(\Lambda_{\ell}(0)\right)$ inside $I_{\Lambda}$.

As one can easily imagine, this description yields the local statistics for both eigenlevels and localization center. Actually as the intervals under consideration are larger than $|\Lambda|^{-1}$, it yields uniform local statistics (see sections 1.7.1 and 1.8.1). Theorem 1.1 is at the heart of the proof the proofs of Theorems 1.9, 1.10, 1.13, 1.14 and 1.15 found in section 1.7 and 1.8. Moreover, under additional the decorrelations estimates (see assumptions (GM) and (D) in section 1.7.2), Theorem 1.1 will be sufficient to prove the mutual independence of the local processes at distinct energies when they are sufficiently far apart, that is, when they are separated by a distance that is asymptotically infinite with respect to $|\Lambda|^{-1}$ (see section 1.7.2). 
1.3.2. Controlling most eigenvalues. Then, we now state a result that works on intervals $I_{\Lambda}$ such that $N\left(I_{\Lambda}\right)$ be of size $(\log |\Lambda|)^{-d / \xi}$ but gives the control only on most of the eigenvalues. This is enough to control the levelspacings on such sets. This is the main tool to obtain Theorems 1.4, 1.6, 1.7 and 1.8. For that purpose we state it in a more axiomatic way than what we did for Theorems 1.1.

Definition 1.1. Pick $\xi \in(0,1), R>1$ large and $\rho^{\prime} \in(0, \rho)$ where $\rho$ is defined in (M). For a cube $\Lambda$, consider an interval $I_{\Lambda}=\left[a_{\Lambda}, b_{\Lambda}\right] \subset I$. Set $\ell_{\Lambda}^{\prime}=(R \log |\Lambda|)^{\frac{1}{\xi}}$. We say that the sequence $\left(I_{\Lambda}\right)_{\Lambda}$ is $\left(\xi, R, \rho^{\prime}\right)$-admissible if, for any $\Lambda$, one has

$$
|\Lambda| N\left(I_{\Lambda}\right) \geq 1, \quad N\left(I_{\Lambda}\right)\left|I_{\Lambda}\right|^{-\left(1+\rho^{\prime}\right)} \geq 1, \quad N\left(I_{\Lambda}\right)^{\frac{1}{1+\rho^{\prime}}}\left(\ell_{\Lambda}^{\prime}\right)^{d} \leq 1
$$

One has

Theorem 1.2. Assume (IAD), (W), (M) and (Loc) hold. Pick $\rho^{\prime} \in[0, \rho /(1+(\rho+1) d))$ where $\rho$ is defined in (M). For any $q>0$, for $L$ sufficiently large, depending only on $\xi, R, \rho^{\prime}, p$, for any sequence of intervals $\left(I_{\Lambda}\right)_{\Lambda}$ that is $\left(\xi, R, \rho^{\prime}\right)$-admissible, and any sequence of scales $\tilde{\ell}_{\Lambda}$ so that $\ell_{\Lambda}^{\prime} \ll \tilde{\ell}_{\Lambda} \ll L$ and

$$
N\left(I_{\Lambda}\right)^{\frac{1}{1+\rho^{\prime}}} \tilde{\ell}_{\Lambda}^{d} \underset{|\Lambda| \rightarrow \infty}{\rightarrow} 0
$$

there exists

- a decomposition of $\Lambda$ into disjoint cubes of the form $\Lambda_{\ell_{\Lambda}}\left(\gamma_{j}\right):=\gamma_{j}+\left[0, \ell_{\Lambda}\right]^{d}$, where $\ell_{\Lambda}=$ $\tilde{\ell}_{\Lambda}\left(1+\mathcal{O}\left(\tilde{\ell}_{\Lambda} /|\Lambda|\right)\right)=\tilde{\ell}_{\Lambda}(1+o(1))$ such that

$-\cup_{j} \Lambda_{\ell_{\Lambda}}\left(\gamma_{j}\right) \subset \Lambda$

$-\operatorname{dist}\left(\Lambda_{\ell_{\Lambda}}\left(\gamma_{j}\right), \Lambda_{\ell_{\Lambda}}\left(\gamma_{k}\right)\right) \geq \ell_{\Lambda}^{\prime}$ if $j \neq k$,

$-\operatorname{dist}\left(\Lambda_{\ell_{\Lambda}}\left(\gamma_{j}\right), \partial \Lambda\right) \geq \ell_{\Lambda}^{\prime}$

$-\left|\Lambda \backslash \cup_{j} \Lambda_{\ell_{\Lambda}}\left(\gamma_{j}\right)\right| \lesssim|\Lambda| \ell_{\Lambda}^{\prime} / \ell_{\Lambda}$,

- a set of configurations $\mathcal{Z}_{\Lambda}$ such that

$-\mathcal{Z}_{\Lambda}$ is large, namely,

$$
\left.\mathbb{P}\left(\mathcal{Z}_{\Lambda}\right) \geq 1-|\Lambda|^{-q}-\exp \left(-c\left|I_{\Lambda}\right|^{1+\rho}|\Lambda| \ell_{\Lambda}^{d \rho}\right)\right)-\exp \left(-c|\Lambda|\left|I_{\Lambda}\right| \ell_{\Lambda}^{\prime} \ell_{\Lambda}^{-1}\right)
$$

so that

- for $\omega \in \mathcal{Z}_{\Lambda}$, there exists at least $\frac{|\Lambda|}{\ell_{\Lambda}^{d}}\left(1+o\left(N\left(I_{\Lambda}\right)^{\frac{1}{1+\rho^{\prime}}} \ell_{\Lambda}^{d}\right)\right)$ disjoint boxes $\Lambda_{\ell_{\Lambda}}\left(\gamma_{j}\right)$ satisfying the properties (1), (2) and (3) described in Theorem 1.1 with $\ell_{\Lambda}^{\prime}=(R \log |\Lambda|)^{1 / \xi}$ in (1.12); we note that $N\left(I_{\Lambda}\right) \ell_{\Lambda}^{d-1} \ell_{\Lambda}^{\prime}=o(1)$ as $|\Lambda| \rightarrow+\infty$;

- the number of eigenvalues of $H_{\omega}(\Lambda)$ that are not described above is bounded by

$$
C N\left(I_{\Lambda}\right)|\Lambda|\left(N\left(I_{\Lambda}\right)^{\frac{\rho-\rho^{\prime}}{1+\rho^{\prime}}} \ell_{\Lambda}^{d(1+\rho)}+N\left(I_{\Lambda}\right)^{-\frac{\rho^{\prime}}{1+\rho^{\prime}}}\left(\ell_{\Lambda}^{\prime}\right)^{d+1} \ell_{\Lambda}^{-1}\right)
$$

this number is $o\left(N\left(I_{\Lambda}\right)|\Lambda|\right)$ provided

$$
N\left(I_{\Lambda}\right)^{-\frac{\rho^{\prime}}{1+\rho^{\prime}}}\left(\ell_{\Lambda}^{\prime}\right)^{d+1} \ll \ell_{\Lambda} \ll N\left(I_{\Lambda}\right)^{-\frac{\rho-\rho^{\prime}}{d(1+\rho)\left(1+\rho^{\prime}\right)}} .
$$

Before turning to the description of the statistics global and local, let us make two remarks about the choice of parameters and length scales for which Theorem 1.2 is useful. First, if $N\left(I_{\Lambda}\right)^{-1}$ is of an order much larger that of $\left(\ell_{\Lambda}^{\prime}\right)^{d}$, then, the condition (1.17) essentially imposes that $\frac{\rho-\rho^{\prime}}{\left(1+\rho^{\prime}\right)(1+\rho)}>\frac{d \rho^{\prime}}{1+\rho^{\prime}}$ which is satisfied if $\rho^{\prime} \in[0, \rho /(1+(\rho+1) d))$. Condition $(1.17)$ then 
guarantees that the condition (1.14) is met: indeed, $\tilde{\ell}_{\Lambda} \sim \ell_{\Lambda}$ and, as $\rho-\rho^{\prime}<1+\rho$ and $N\left(I_{\Lambda}\right) \rightarrow 0$, one has

$$
N\left(I_{\Lambda}\right)^{-1 /\left(1+\rho^{\prime}\right)} \ll N\left(I_{\Lambda}\right)^{-\frac{\rho-\rho^{\prime}}{(1+\rho)\left(1+\rho^{\prime}\right)}} .
$$

We shall use this in the proof of the large deviation estimate (1.21) in conjunction with a choice of $\ell_{\Lambda}$ and $N\left(I_{\Lambda}\right)^{-1}$ as large powers of $\ell_{\Lambda}^{\prime}$, that is, large powers of $\log |\Lambda|$ (see section 2.2).

Note that, if $\rho^{\prime} \in[0, \rho /(1+d(\rho+1)))$, then, (1.17) and (1.14) are satisfied for some choice of $\alpha \in(0,1)$ and $\nu \in(0,1 / d)$ if one sets $\ell_{\Lambda}^{\prime} \asymp(\log |\Lambda|)^{1 / \xi}, \ell_{\Lambda} \asymp N\left(I_{\Lambda}\right)^{-\nu}$ and $N\left(I_{\Lambda}\right) \asymp|\Lambda|^{-\alpha}$ (see section 4.3.1). This is the choice of parameters we shall use in our study of level spacings.

In [GK112], for a less general class of models, by improving on the assumptions (W) and (M), we shall improve on the bound (1.16), which

- will enable us to relax the second condition in (1.13) so as to admit $N\left(I_{\Lambda}\right)$ of size $e^{-\left|I_{\Lambda}\right|^{-\alpha}}$ for $\alpha \in(0,1)$;

- will provide a better large deviation estimate for the number of eigenvalues of $H_{\omega}(\Lambda)$ in the interval $I_{\Lambda}$ than the first rough estimate given in Theorem 1.3.

1.3.3. Comparing various cube sizes. We end this section with a related result we shall use in the sequel. We prove

Proposition 1.1. Assume (IAD) (Loc) and (W) in J. Fix $I \subset J$ a compact interval and $C, C^{\prime}$, compact cubes in $\mathbb{R}^{d}$ such that $\bar{C} \subset \stackrel{\circ}{C^{\prime}}$. Fix $p>0$ and a sequence $\left(\ell_{\Lambda}\right)_{\Lambda}$ satisfying (1.52). Then, there exists a set of configurations $\mathcal{Z}_{\Lambda}$ so that $\mathbb{P}\left(\mathcal{Z}_{\Lambda}\right) \rightarrow 1$ as $|\Lambda| \rightarrow+\infty$ and, for $\omega \in \mathcal{Z}_{\Lambda}$ and $|\Lambda|$ sufficiently large,

- to every eigenvalue of $H_{\omega}(\Lambda)$ in $E_{0}+\ell_{\Lambda}^{-d} I$ associated to a localization center in $\ell_{\Lambda} C$, say $E_{j}(\omega, \Lambda)$, one can associate an eigenvalue of $H_{\omega}\left(\ell_{\Lambda} C^{\prime}\right)$, say $E_{j}\left(\omega, \ell_{\Lambda} C^{\prime}\right)$; moreover, these eigenvalues satisfy

$$
\left|E_{j}\left(\omega, \ell_{\Lambda} C^{\prime}\right)-E_{j}(\omega, \Lambda)\right| \leq|\Lambda|^{-p}
$$

- to every eigenvalue of $H_{\omega}\left(\ell_{\Lambda} C^{\prime}\right)$ in $E_{0}+\ell_{\Lambda}^{-d} I$ associated to a localization center in $\ell_{\Lambda} C$, say $E_{j}\left(\omega, \ell_{\Lambda} C^{\prime}\right)$, one can associate an eigenvalue of $H_{\omega}(\Lambda)$, say $E_{j}(\omega, \Lambda)$ with localization center in $\ell_{\Lambda} C^{\prime}$; moreover, these eigenvalues satisfy

$$
\left|E_{j}\left(\omega, \ell_{\Lambda} C^{\prime}\right)-E_{j}(\omega, \Lambda)\right| \leq|\Lambda|^{-p} .
$$

Proposition 1.1 is a consequence of Lemma 3.1.

Remark 1.5. As the proofs will show, the sizes of the intervals where the control of the eigenvalues is possible and the probability of the event where this control is possible both depend very much on the forms of the Wegner and Minami estimates, (W) and (M). In particular, if one replaces (W) by what is suggested in Remark 1.1, the constants appearing in Theorems 1.1 and 1.2 and Proposition 1.1 have to be modified.

1.4. The level spacings statistics. Our goal is now to understand the level spacings statistics for eigenvalues near $E_{0} \in I$. Pick $I_{\Lambda}$ a compact interval containing $E_{0}$ such that its density of states measure $N\left(I_{\Lambda}\right)$ stays bounded. We note that, by the existence of the density of states and also Theorem 1.9, the spacing between the image of the eigenvalues of $H_{\omega}(\Lambda)$ through $N$ near $E_{0}$ is of size $|\Lambda|^{-1}$. Hence, to study the empirical statistics of level spacings in $I_{\Lambda}, N\left(I_{\Lambda}\right)$ should contain asymptotically infinitely many images of energy levels of $H_{\omega}(\Lambda)$. Let us first study this number. 
1.4.1. A large deviation principle for the eigenvalue counting function. Define the random numbers

$$
N\left(I_{\Lambda}, \Lambda, \omega\right):=\#\left\{j ; E_{j}(\omega, \Lambda) \in I_{\Lambda}\right\} .
$$

Write $I_{\Lambda}=\left[a_{\Lambda}, b_{\Lambda}\right]$ and recall that $N\left(I_{\Lambda}\right)=N\left(b_{\Lambda}\right)-N\left(a_{\Lambda}\right)$ where $N$ is the integrated density of states. We show that $N\left(I_{\Lambda}, \Lambda, \omega\right)$ satisfies a large deviation principle, namely,

Theorem 1.3. Assume (IAD), (W), (M) and (Loc) hold. Fix $\tilde{\rho} \in(0, \rho /(1+d(\rho+1)))$ where $\rho$ is given by $(M)$, and $\nu \in(0,1)$. Then, there exists $\delta>0$ small such that, if $\left(I_{\Lambda}\right)_{\Lambda}$ is a sequence of compact intervals in the localization region I satisfying

- $N\left(I_{\Lambda}\right)(\log |\Lambda|)^{1 / \delta} \rightarrow 0$ as $|\Lambda| \rightarrow+\infty$

- $N\left(I_{\Lambda}\right)|\Lambda|^{1-\nu} \rightarrow+\infty$ as $|\Lambda| \rightarrow+\infty$

- $N\left(I_{\Lambda}\right)\left|I_{\Lambda}\right|^{-1-\tilde{\rho}} \rightarrow+\infty$ as $|\Lambda| \rightarrow+\infty$,

then, for any $p>0$, for $|\Lambda|$ sufficiently large (depending on $\rho^{\prime}$ and $\nu$ but not on the specific sequence $\left.\left(I_{\Lambda}\right)_{\Lambda}\right)$, one has

$$
\mathbb{P}\left(\left|N\left(I_{\Lambda}, \Lambda, \omega\right)-N\left(I_{\Lambda}\right)\right| \Lambda|| \geq N\left(I_{\Lambda}\right)|\Lambda|(\log |\Lambda|)^{-\delta}\right) \leq|\Lambda|^{-p} .
$$

We note that we do not need that intervals $\left(I_{\Lambda}\right)_{\Lambda}$ lie near points $E_{0}$ where (1.42) is satisfied; the density of states may vanish near $E_{0}$ though not faster than the rate fixed by the condition $N\left(I_{\Lambda}\right)\left|I_{\Lambda}\right|^{-1-\rho} \rightarrow+\infty$. The large deviation principle (1.21) is meaningful only if $N\left(I_{\Lambda}\right)|\Lambda| \rightarrow+\infty$; as $N$ is Lipschitz continuous as a consequence of $(\mathrm{W})$, this implies that

$$
|\Lambda| \cdot\left|I_{\Lambda}\right| \rightarrow+\infty \quad \text { when } \quad|\Lambda| \rightarrow+\infty .
$$

For the discrete Anderson model, we improve upon (1.21) in [GKl12] by relaxing $N\left(I_{\Lambda}\right)\left|I_{\Lambda}\right|^{-1-\rho} \rightarrow$ $+\infty$ into $N\left(I_{\Lambda}\right)\left|I_{\Lambda}\right|^{-\nu} \rightarrow+\infty$ for some arbitrarily large $\nu>0$ and obtain precise estimates of the term $o\left(N\left(I_{\Lambda}\right)|\Lambda|\right)$, exploiting an improved Wegner and Minami estimate.

1.4.2. The level spacing statistics near a given energy. Fix $E_{0} \in I$. Pick $I_{\Lambda}=\left[a_{\Lambda}, b_{\Lambda}\right]$ so that $\left|a_{\Lambda}\right|+\left|b_{\Lambda}\right| \rightarrow 0$. Consider the unfolded eigenvalue spacings, for $1 \leq j \leq N$,

$$
\delta N_{j}(\omega, \Lambda)=|\Lambda|\left(N\left(E_{j+1}(\omega, \Lambda)\right)-N\left(E_{j}(\omega, \Lambda)\right)\right) \geq 0 .
$$

Define the empirical distribution of these spacings to be the random numbers, for $x \geq 0$

$$
D L S\left(x ; E_{0}+I_{\Lambda}, \omega, \Lambda\right)=\frac{\#\left\{j ; E_{j}(\omega, \Lambda) \in E_{0}+I_{\Lambda}, \delta N_{j}(\omega, \Lambda) \geq x\right\}}{N\left(I_{\Lambda}, \Lambda, \omega\right)} .
$$

We will now study the spacings distributions of energies inside intervals that shrink to a point but that asymptotically contain infinitely many eigenvalues.

We prove

Theorem 1.4. Assume (IAD), (W), (M) and (Loc) hold. Fix $E_{0} \in I$ such that, for some $\tilde{\rho} \in$ $[0, \rho /(1+d(\rho+1)))$, there exists a neighborhood of $E_{0}$, say, $U$ such that

$$
\forall(x, y) \in U^{2}, \quad|N(x)-N(y)| \geq|x-y|^{1+\tilde{\rho}} .
$$

Fix $\left(I_{\Lambda}\right)_{\Lambda}$ a decreasing sequence of intervals such that $\sup _{E \in I_{\Lambda}}|E| \underset{|\Lambda| \rightarrow+\infty}{\rightarrow} 0$.

Assume that, for some $\delta>0$, one has

$$
|\Lambda|^{1-\delta} \cdot N\left(E_{0}+I_{\Lambda}\right) \underset{|\Lambda| \rightarrow+\infty}{\rightarrow}+\infty \text { and if } \ell^{\prime}=o(L) \text { then } \frac{N\left(E_{0}+I_{\Lambda_{L+\ell^{\prime}}}\right)}{N\left(E_{0}+I_{\Lambda_{L}}\right)} \underset{|\Lambda| \rightarrow+\infty}{\rightarrow} 1 \text {. }
$$

Then, with probability 1 , as $|\Lambda| \rightarrow+\infty, D L S\left(x ; E_{0}+I_{\Lambda}, \omega, \Lambda\right)$ converges uniformly to the distribution $x \mapsto e^{-x}$, that is, with probability 1 ,

$$
\sup _{x \geq 0}\left|D L S\left(x ; E_{0}+I_{\Lambda}, \omega, \Lambda\right)-e^{-x}\right| \underset{|\Lambda| \rightarrow+\infty}{\rightarrow} 0 .
$$


Hence, the unfolded level spacings behave as if the images of the eigenvalues by the IDS were i.i.d. uniformly distributed random variables (see [Wei55] or section 7 of [Pyk65]). The exponential distribution of the level spacings is the one predicted by physical heuristics in the localized regime ([Jan98, LR85, Mir00, Tho74]). It is also in accordance with Theorem 1.9. In [Mol82, Min96], the domains in energy where the statistics were studied were much smaller than the ones considered in Theorem 1.4. Indeed, in these works, the energy interval is of order $|\Lambda|^{-1}$ whereas, here, it is assumed to tend to 0 but to be asymptotically infinite when compared to $|\Lambda|^{-1}$.

Remark 1.6. The first condition in (1.26) ensures that $I_{\Lambda}$ contains sufficiently many eigenvalues of $H_{\omega}(\Lambda)$. The second condition in (1.26) is a regularity condition of the decay of $\left|I_{\Lambda}\right|$.

If, in (1.26), one replaces the first condition by $|\Lambda| N\left(E_{0}+I_{\Lambda}\right) \rightarrow+\infty$ or omits the second or does both, one still gets convergence in probability of $D L S\left(x ; E_{0}+I_{\Lambda}, \omega, \Lambda\right)$ to $e^{-x}$ (see Remark 4.3) i.e.

$$
\mathbb{P}\left(\sup _{x \geq 0}\left|D L S\left(x ; E_{0}+I_{\Lambda}, \omega, \Lambda\right)-e^{-x}\right| \geq \varepsilon\right) \underset{|\Lambda| \rightarrow+\infty}{\rightarrow} 0 .
$$

Condition (1.25) is slightly stronger than (1.42); it requires some uniformity in the lower bound. Theorem 1.4 can be applied to obtain the levelspacing distribution near regular points of the IDS. Define $\mathcal{E}$ to be the set of energies $E$ such that $\nu(E)=N^{\prime}(E)$ exists and

$$
\lim _{|x|+|y| \rightarrow 0} \frac{N(E+x)-N(E+y)}{x-y}=\nu(E) .
$$

Obviously the set $\mathcal{E}$ contains the continuity points of $\nu(E)$. We prove in Appendix

Lemma 1.3. The set $\mathcal{E}$ is of full Lebesgue measure.

For $E_{0} \in \mathcal{E}$ such that $\nu\left(E_{0}\right)>0$, assumption (1.25) holds with $\tilde{\rho}=0$. Moreover, one has

$$
\delta E_{j}(\omega, \Lambda)=\nu\left(E_{0}\right)|\Lambda|\left(E_{j+1}(\omega, \Lambda)-E_{j}(\omega, \Lambda)\right)(1+o(1)) .
$$

Then as a corollary of Theorem 1.4, we immediately obtain

Theorem 1.5. Assume (IAD), (W), (M) and (Loc) hold. Fix $E_{0} \in I \cap \mathcal{E}$ such that $\nu\left(E_{0}\right)>0$. Fix $\left(I_{\Lambda}\right)_{\Lambda}$ a sequence of intervals such that $\sup _{I_{\Lambda}}|x| \underset{|\Lambda| \rightarrow+\infty}{\rightarrow} 0$.

Assume that, for some $\delta>0$, one has

$$
|\Lambda|^{1-\delta} \cdot\left|I_{\Lambda}\right| \underset{|\Lambda| \rightarrow+\infty}{\rightarrow}+\infty \text { and if } \ell^{\prime}=o(L) \text { then } \frac{\left|I_{\Lambda_{L+\ell^{\prime}}}\right|}{\left|I_{\Lambda_{L}}\right|} \underset{|\Lambda| \rightarrow+\infty}{\rightarrow} 1 .
$$

Then, with probability 1 , as $|\Lambda| \rightarrow+\infty$, the empirical distribution function

$$
\frac{\#\left\{j ; E_{j}(\omega, \Lambda) \in I_{\Lambda}, \nu\left(E_{0}\right)|\Lambda|\left(E_{j+1}(\omega, \Lambda)-E_{j}(\omega, \Lambda)\right) \geq x\right\}}{N\left(I_{\Lambda}, \Lambda, \omega\right)}
$$

converges uniformly to the distribution $x \mapsto e^{-x}$.

1.4.3. The level spacings statistics on macroscopic energy intervals. Theorem 1.5 seems optimal as the density of states at $E_{0}$ enters into the correct rescaling to obtain a universal result. Hence, the distribution of level spacings on larger intervals needs to take into account the variations of the density of states on these intervals. Indeed, on intervals of non vanishing size, under additional regularity assumption on $\nu$, one can compute the asymptotic distribution of the level spacings when one omits the local density of states in the spacing and obtain the 
Theorem 1.6. Assume (IAD), (W), (M) and (Loc) hold. Pick $J \subset I$ a compact interval such $\lambda \mapsto \nu(\lambda)$ be continuous on $J$ and $N(J):=\int_{J} \nu(\lambda) d \lambda>0$. Define the unfolded eigenvalue spacings, for $1 \leq j \leq N$,

$$
\delta_{J} E_{j}(\omega, \Lambda)=\frac{N(J)}{|J|}|\Lambda|\left(E_{j+1}(\omega, \Lambda)-E_{j}(\omega, \Lambda)\right) \geq 0,
$$

and the empirical distribution of these spacings to be the random numbers, for $x \geq 0$,

$$
D L S^{\prime}(x ; J, \omega, \Lambda)=\frac{\#\left\{j ; E_{j}(\omega, \Lambda) \in J, \delta_{J} E_{j}(\omega, \Lambda) \geq x\right\}}{N(J, \omega, \Lambda)} .
$$

Then, with probability 1 , as $|\Lambda| \rightarrow+\infty, D L S^{\prime}(x ; J, \omega, \Lambda)$ converges uniformly to the distribution $x \mapsto g_{\nu, J}(x)$ where

$$
g_{\nu, J}(x)=\int_{J} \mathrm{e}^{-\nu_{J}(\lambda)|J| x} \nu_{J}(\lambda) d \lambda \text { where } \nu_{J}=\frac{1}{N(J)} \nu .
$$

We see that, in the large volume limit, the unfolded levelspacings behave as if the eigenvalues were i.i.d. random variables distributed according to the density $\frac{1}{N(J)} \nu(\lambda)$ i.e. to the density of states renormalized to be a probability measure on $J$ (see section 7 of [Pyk65]).

Theorem 0.1 is then an immediate consequence of Theorem 1.6 and the results on the regularity for the density of states of the discrete Anderson model at large disorder obtained in [BCKP88]. We point out that for random Hamiltonians in the continuum, the continuity of the density of states is still an open problem.

1.5. The localization center spacings statistics. Pick $E_{0} \in I$. Inside a large cube $\Lambda$, the number of centers that corresponds to energies in $I_{\Lambda}, N\left(E_{0}+I_{\Lambda}, \Lambda, \omega\right)$ (see (1.20)), is asymptotic to $N\left(E_{0}+I_{\Lambda}\right)|\Lambda|$. Theorem 1.15 states that they are distributed uniformly. Thus, the reference mean spacing between localization centers is of size $\left(|\Lambda| / N\left(E_{0}+I_{\Lambda}\right)|\Lambda|\right)^{1 / d}=\left(N\left(E_{0}+I_{\Lambda}\right)\right)^{-1 / d}$. This motivates the following definition. Define the empirical distribution of center spacing to be the random number

$$
D C S\left(s ; I_{\Lambda}, \Lambda, \omega\right)=\frac{\#\left\{j ; \sqrt[d]{N\left(E_{0}+I_{\Lambda}\right)} \min _{i \neq j}\left|x_{i}(\omega)-x_{j}(\omega)\right| \geq s\right\}}{N\left(E_{0}+I_{\Lambda}, \Lambda, \omega\right)} .
$$

We prove an analogue of Theorem 1.4, namely

Theorem 1.7. Assume (IAD), (W), (M) and (Loc) hold. Pick $E_{0} \in I$ such that, for some $\tilde{\rho} \in[0, \rho /(1+d(\rho+1)))$ small enough (depending on $\rho$ and $d)$, in $U$ some neighborhood of $E_{0}$, one has (1.25). Assume that, for some $\nu \in(0,1)$, one has

$$
N\left(E_{0}+I_{\Lambda}\right)|\Lambda| \underset{|\Lambda| \rightarrow+\infty}{\rightarrow}+\infty \text { and } N\left(E_{0}+I_{\Lambda}\right) \log ^{d / \nu}|\Lambda| \underset{|\Lambda| \rightarrow+\infty}{\rightarrow} 0 .
$$

Then, as $|\Lambda| \rightarrow+\infty$, in probability, DCS $\left(s ; I_{\Lambda}, \Lambda, \omega\right)$ converges uniformly to the distribution $x \mapsto$ $e^{-s^{d}}$, that is, for any $\varepsilon>0$,

$$
\mathbb{P}\left(\left\{\omega ; \sup _{s \geq 0}\left|D C S\left(s ; E_{0}+I_{\Lambda}, \Lambda, \omega\right)-e^{-s^{d}}\right| \geq \varepsilon\right\}\right) \underset{\Lambda \nearrow \mathbb{R}^{d}}{\rightarrow} 0 .
$$

Of course, as Theorem 1.7 is the counterpart of Theorem 1.4, Theorem 1.6 also has its counterpart for localization centers. 
1.6. Another point of view. In the present section, we want to adopt a different point of view on the spectral statistics. Instead of discussing the statistics of the eigenvalues of the random system restricted to some finite box in the large box limit, we will describe the spectral statistics of the infinite system in the localized phase. Therefore, we first need to explicit what we mean with the localized phase for the random Hamiltonian on the whole space i.e. state the appropriate replacement for assumption (Loc) in this setting.

Let $I \subset \mathbb{R}$ be an interval. We assume

(Loc'): there exists $\xi \in(0,1], q>0$ and $\gamma>0$ such that, with probability 1 , if $E \in I \cap \sigma\left(H_{\omega}\right)$ and $\varphi$ is a normalized eigenfunction associated to $E$ then, for some $x(E, \omega) \in \mathbb{R}^{d}$ or $\mathbb{Z}^{d}$, a maximum of $x \mapsto\|\varphi\|_{x}$, for some $C_{\omega}>0$, one has, for $x \in \mathbb{R}^{d}$,

$$
\|\varphi\|_{x} \leq C_{\omega}\left(1+|x(E, \omega)|^{2}\right)^{q / 2} e^{-\gamma|x-x(E, \omega)|^{\xi}} ;
$$

moreover, one has $\mathbb{E}\left(C_{\omega}\right)<+\infty$.

As above $x(E)$ is called a center of localization for energy $E$ or for the associated eigenfunction $\varphi$. It is well established that (Loc') holds in any interval contained in the region of complete localization. The first proof is due to [dRJLS96] for the discrete Anderson model where they show that (Loc') is a consequence of the fractional moment method [Aiz94, AM93]; there $\xi=1$. The proof extends to continuous Hamiltonians thanks to [AEN $\left.{ }^{+} 06\right]$. In [GDB98], the multiscale analysis is shown to imply (1.35) with $\xi=1$ but with no control on $\mathbb{E}\left(C_{\omega}\right)$. That the multiscale analysis yields (Loc') for any $\xi<1$ follows from [GK06, Corollary 3] and [GK06, Eq. (4.17)] to see that $\mathbb{E}\left(C_{\omega}\right)<\infty$.

Pick $I$ an interval where the Hamiltonian $H_{\omega}$ is localized i.e. satisfies (Loc'). Assume that, $\omega$-almost surely, $\sigma\left(H_{\omega}\right) \cap I=I$. Hence, any subinterval of $I$ contains infinitely many eigenvalues and to define statistics, we need to enumerate these eigenvalues in a way or another. To do this, we use the localization centers. First, we prove

Proposition 1.2. Assume (Loc') for some $\xi \in(0,1]$ and fix $q>2 d$. Then, there exists $\gamma>0$ such that, $\omega$-almost surely, there exists $C_{\omega}>1, \mathbb{E}\left(C_{\omega}\right)<\infty$, such that

(1) if $x(E, \omega)$ and $x^{\prime}(E, \omega)$ are two centers of localization for $E \in I$ then, for some $C_{d}>0$ (depending only on $d$ ),

$$
\left|x(E, \omega)-x^{\prime}(E, \omega)\right| \leq \gamma^{-1 / \xi} \log ^{1 / \xi}\left(C_{d} C_{\omega}\langle x(E, \omega)\rangle^{q}\left\langle x^{\prime}(E, \omega)\right\rangle^{q} \frac{1}{\gamma^{d / \xi}}\right) .
$$

(2) for $L \geq 1$, pick $\left(I_{L}\right)_{L}$ a sequence of intervals, $I_{L} \subset I$, such that, for some $\varepsilon>0$, one has $L^{d-\varepsilon} N\left(I_{L}\right) \rightarrow+\infty$ and $N\left(I_{L}\right)\left|I_{L}\right|^{-1-\rho} \rightarrow+\infty$ where $\rho$ is given by $(\mathrm{M})$ and $N\left(I_{L}\right)$ by (1.4); if $N\left(I_{L}, L\right)$ denotes the number of eigenvalues of $H_{\omega}$ having a center of localization in $\Lambda_{L}$, then

$$
N\left(I_{L}, L\right)=N\left(I_{L}\right)\left|\Lambda_{L}\right|(1+o(1)) .
$$

For $L \geq 1$, pick $I_{L} \subset I$ such that $L^{d-\varepsilon} N\left(I_{L}\right) \rightarrow+\infty$ for some $\varepsilon>0$. In view of Proposition 1.2, there are only finitely many eigenvalues of $H_{\omega}$ in $I_{L}$ having a localization center in $\Lambda_{L}$. Thus, we can consider their levelspacings: let us enumerate these eigenvalues as $E_{1}(\omega, L) \leq E_{2}(\omega, L) \leq$ $\cdots \leq E_{N}(\omega, L)$ where we repeat them according to multiplicity. Define the empirical distributions $D L S$ and $D L S^{\prime}$ as in (1.24) and (1.30) for the eigenvalues of $H_{\omega}$ in $I_{L}$ having a localization center in $\Lambda_{L}$. We prove

Theorem 1.8. Assume (IAD), (W), (M), (Loc') hold. One has 
- if $E_{0} \in I_{L}$ s.t. (1.42) is satisfied for some $\tilde{\rho} \in[0, \rho /(1+d \rho))$ and $\left|I_{L}\right| \rightarrow 0$ and satisfies (1.26), then, $\omega$-almost surely, for $x \geq 0$,

$$
\lim _{L \rightarrow+\infty} D L S\left(x ; I_{L}, \omega, L\right)=e^{-x} ;
$$

- if, for all $L$ large, $\left|I_{L}\right|=J$ such that $N(J)>0$ and $\nu$ is continuous on $J$ then, $\omega$-almost surely, one has

$$
\lim _{L \rightarrow+\infty} D L S^{\prime}\left(x ; I_{L}, \omega, L\right)=g_{J}(x),
$$

where $g_{J}$ is defined in (1.31).

We see that the level spacings distribution of the eigenvalues of $H_{\omega}$ having center of localization in $\Lambda_{L}$ have the same limits as those of the eigenvalues of $H_{\omega}(\Lambda)$. This is a consequence of the localization assumption (Loc').

1.7. The local level statistics. We now exploit of approximation of eigenvalues by iid ones to revisit and extend previous results on the convergence to the Poisson law of rescaled eigenvalues and centers of localization.

For $L \in \mathbb{N}$, recall that $\Lambda=\Lambda_{L}$ and that $H_{\omega}(\Lambda)$ is the operator $H_{\omega}$ restricted to $\Lambda$ with periodic boundary conditions. The notation $|\Lambda| \rightarrow+\infty$ is a shorthand for considering $\Lambda=\Lambda_{L}$ in the limit $L \rightarrow+\infty$.

Denote the eigenvalues of $H_{\omega}(\Lambda)$ ordered increasingly and repeated according to multiplicity by $E_{1}(\omega, \Lambda) \leq E_{2}(\omega, \Lambda) \leq \cdots \leq E_{N}(\omega, \Lambda) \leq \cdots$.

Let $E_{0}$ be an energy in $I$. The unfolded local level statistics near $E_{0}$ is the point process defined by

$$
\Xi\left(\xi ; E_{0}, \omega, \Lambda\right)=\sum_{j \geq 1} \delta_{\xi_{j}\left(E_{0}, \omega, \Lambda\right)}(\xi)
$$

where

$$
\xi_{j}\left(E_{0}, \omega, \Lambda\right)=|\Lambda|\left(N\left(E_{j}(\omega, \Lambda)\right)-N\left(E_{0}\right)\right) .
$$

The numbers $\left(|\Lambda| N\left(E_{j}(\omega, \Lambda)\right)\right)_{j}$ are called the unfolded eigenvalues of $H_{\omega}(\Lambda)$ (see e.g. [Min07, Min08] for more details).

The unfolded local level statistics are described by

Theorem 1.9. Assume (IAD), (W), (M) and (Loc) hold. Pick $\tilde{\rho}$ satisfying (1.10) where $\rho$ is defined by $(M)$.

Pick $E_{0}$ be an energy in I such that the integrated density of states satisfies

$$
\forall a>b, \exists C(a, b)>0, \exists \varepsilon_{0}>0, \forall \varepsilon \in\left(0, \varepsilon_{0}\right), \quad\left|N\left(E_{0}+a \varepsilon\right)-N\left(E_{0}+b \varepsilon\right)\right| \geq C(a, b) \varepsilon^{1+\tilde{\rho}} .
$$

When $|\Lambda| \rightarrow+\infty$, the point process $\Xi\left(E_{0}, \omega, \Lambda\right)$ converges weakly to a Poisson process on $\mathbb{R}$ with intensity the Lebesgue measure.

If one assume that $N$ is differentiable at $E_{0}$ and that its derivative $\nu\left(E_{0}\right)$ is positive i.e.

$$
0<\nu\left(E_{0}\right):=\lim _{E \rightarrow E_{0}} \frac{N(E)-N\left(E_{0}\right)}{E-E_{0}},
$$

it is easy to check that (1.42) is satisfied with $\tilde{\rho}=0$ and that, for $E_{j}-E_{0}$ small,

$$
\xi_{j}\left(E_{0}, \omega, \Lambda\right)=|\Lambda| \nu\left(E_{0}\right)\left(E_{j}(\omega, \Lambda)-E_{0}\right)(1+o(1)) .
$$

Thus, one recovers the convergence to a Poisson process when the point process (1.40) is replaced by the one defined by the points $\left(|\Lambda| \nu\left(E_{0}\right)\left(E_{j}(\omega, \Lambda)-E_{0}\right)\right)_{j}$.

Theorem 1.9 under the additional assumption (1.43) was first obtained in [Mol82] for a special one dimensional random Schrödinger model on the real line. For the discrete Anderson model, 
Theorem 1.8 was proved in [Min96] under the assumption (1.43).

Our method of proof is different from that of [Min96] and, in spirit, closer to that of [Mol82] and to the physical heuristics. Clearly, for (1.42) to be satisfied, we do not need $N$ to be differentiable at $E_{0}$ nor its derivative to be positive. E.g. if $N$ satisfies $N(E)=N\left(E_{0}\right)+c\left(E-E_{0}\right)^{1+\tilde{\rho}}(1+o(1))$ near $E_{0}$ then (1.42) is satisfied. Condition (1.42) asks that at a given scale, $N$ behaves roughly uniformly near $E_{0}$. Note however that, if $N$ is not differentiable at $E_{0}$, then the local statistics of the eigenvalues themselves will not be Poissonian anymore.

Our method yields a uniform version of Theorem 1.9 to which we now turn.

1.7.1. Uniform Poisson convergence over small intervals. Fix $\alpha \in\left(\alpha_{d, \rho, \tilde{\rho}}, 1\right)$ (recall that $\alpha_{d, \rho, \tilde{\rho}}$ is defined in (1.11)). The uniform version of the Poisson process is a version that holds uniformly over an interval of energy, say, $I$ centered at $E_{0}$ such that $N(I) \asymp|\Lambda|^{-\alpha}$. Such an interval is much larger than an interval satisfying $N(I) \asymp|\Lambda|^{-1}$. This is the main improvement of Theorem 1.10 below over Theorem 1.9 or the statements found in [KN07, Min96, Mol82]. It is natural to wonder what is the largest size of interval in which a result like Theorem 1.10. We do not know the answer to that question.

Let $I_{\Lambda}\left(E_{0}, \alpha\right)$ be the interval such that $N\left(I_{\Lambda}\left(E_{0}, \alpha\right)\right)$ be centered at $N\left(E_{0}\right)$ of length $2|\Lambda|^{-\alpha}$. Denote by $N_{\Lambda}\left(\omega, E_{0}\right):=\operatorname{tr} \mathbf{1}_{I_{\Lambda}\left(E_{0}, \alpha\right)}\left(H_{\omega}(\Lambda)\right)$ the number of eigenvalues of $H_{\omega}(\Lambda)$ in $I_{\Lambda}\left(E_{0}, \alpha\right)$. For $1 \leq j \leq N_{\Lambda}\left(\omega, E_{0}\right)$, define the unfolded local eigenvalues $\xi_{j}(\omega, \Lambda)$ by (1.41). Hence, for all $1 \leq j \leq N_{\Lambda}\left(\omega, E_{0}\right)$, one has $\xi_{j}(\omega, \Lambda) \in|\Lambda|^{1-\alpha} \cdot[-1,1]$.

We then prove

Theorem 1.10. Assume (IAD), (W), (M) and (Loc) hold. Let $E_{0}$ be an energy in I such that, for some $\tilde{\rho}$ such that (1.10) holds true and such that

$$
\tilde{\rho} \geq \rho \frac{1-d \rho}{1+d \rho}
$$

the integrated density of states satisfies

$$
\forall \delta \in(0,1), \exists C(\delta)>0, \exists \varepsilon_{0}>0, \forall \varepsilon \in\left(0, \varepsilon_{0}\right), \forall a \in[-1,1],
$$

$$
\left|N\left(E_{0}+(a+\delta) \varepsilon\right)-N\left(E_{0}+a \varepsilon\right)\right| \geq C(\delta) \varepsilon^{1+\tilde{\rho}} .
$$

Pick $\alpha \in\left(\alpha_{d, \rho, \tilde{\rho}}, 1\right)$. Then, there exists $\delta>0$ such that, for any sequence of intervals $I_{1}=$ $I_{1}^{\Lambda}, \ldots, I_{p}=I_{p}^{\Lambda}$ in $|\Lambda|^{1-\alpha} \cdot[-1,1]$ (here, $p$ may depend on $\Lambda$ and be arbitrarily large) satisfying

$$
\inf _{j \neq k} \operatorname{dist}\left(I_{j}, I_{k}\right) \geq \mathrm{e}^{-|\Lambda|^{\delta}},
$$

we have, for any sequences of integers $k_{1}=k_{1}^{\Lambda}, \cdots, k_{p}=k_{p}^{\Lambda} \in \mathbb{N}^{p}$,

$$
\lim _{|\Lambda| \rightarrow+\infty}\left|\mathbb{P}\left(\left\{\begin{array}{c}
\#\left\{j ; \xi_{j}(\omega, \Lambda) \in I_{1}\right\}=k_{1} \\
\left.\omega ; \quad \begin{array}{c}
\vdots \\
\#\{j ;
\end{array} \xi_{j}(\omega, \Lambda) \in I_{p}\right\}=k_{p}
\end{array}\right\}\right)-\frac{\left|I_{1}\right|^{k_{1}}}{k_{1} !} \mathrm{e}^{-\left|I_{1}\right|} \cdots \frac{\left|I_{p}\right|^{k_{p}}}{k_{p} !} \mathrm{e}^{-\left|I_{p}\right|}\right|=0 .
$$

In particular, $\Xi\left(\xi ; E_{0}, \omega, \Lambda\right)$ defined in (1.40) converges weakly to a Poisson point process with intensity Lebesgue.

Note that, in Theorem 1.10, we do not require the limits

$$
\begin{aligned}
\lim _{|\Lambda| \rightarrow+\infty} \frac{\left|I_{1}\right|^{k_{1}}}{k_{1} !} \mathrm{e}^{-\left|I_{1}\right|} & =\lim _{|\Lambda| \rightarrow+\infty} \frac{\left|I_{1}^{\Lambda}\right|^{k_{1}^{\Lambda}}}{k_{1}^{\Lambda} !} \mathrm{e}^{-\left|I_{1}^{\Lambda}\right|}, \ldots, \\
\lim _{|\Lambda| \rightarrow+\infty} \frac{\left|I_{p}\right|^{k_{p}}}{k_{p} !} \mathrm{e}^{-\left|I_{p}\right|} & =\lim _{|\Lambda| \rightarrow+\infty} \frac{\left|I_{p}^{\Lambda}\right|_{p}^{k_{p}^{\Lambda}}}{k_{p}^{\Lambda} !} \mathrm{e}^{-\left|I_{p}^{\Lambda}\right|}
\end{aligned}
$$


to exist.

Condition (1.44) imposes no restriction upon condition (1.10) if we know that the Minami estimate (M) holds for all $\rho$ in $(0,1)$. This is the case for all the models we know of for which the Minami estimate is proved (see [Min96, GV07, BHS07, CGK09, K112] and refereces therein).

1.7.2. Asymptotic independence of the local processes. Once Theorem 1.9 is known, it is natural to wonder how the point processes obtained at distinct energies relate to each other. To understand this, we assume

(GM): for $J \subset K \subset I$, one has

$$
\mathbb{E}\left[\operatorname{tr}\left(\mathbf{1}_{J}\left(H_{\omega}(\Lambda)\right)\right) \cdot \operatorname{tr}\left(\mathbf{1}_{K}\left(H_{\omega}(\Lambda)\right)-1\right)\right] \leq C|J||K||\Lambda|^{2} .
$$

(D): for $\beta \in(0,1)$ and $\left\{E_{0}, E_{0}^{\prime}\right\} \subset I$ s.t. $E_{0} \neq E_{0}^{\prime}$, when $L \rightarrow+\infty$ and $\ell \asymp L^{\beta}$, one has

$$
\mathbb{P}\left(\left\{\begin{array}{c}
\sigma\left(H_{\omega}\left(\Lambda_{\ell}\right)\right) \cap\left(E_{0}+L^{-d}[-1,1]\right) \neq \emptyset, \\
\sigma\left(H_{\omega}\left(\Lambda_{\ell}\right)\right) \cap\left(E_{0}^{\prime}+L^{-d}[-1,1]\right) \neq \emptyset
\end{array}\right\}\right)=o\left((\ell / L)^{d}\right) .
$$

In their nature, assumptions (GM) and (D) are similar: they state that the probability to have two eigenvalues constrained to some intervals is much smaller than that of having a single eigenvalue in an interval. Note that $(\ell / L)^{d}$ is the order of magnitude of the right hand side in Wegner's estimate (W) for $H_{\omega}\left(\Lambda_{\ell}\right)$ and the interval $E_{0}+L^{-d}[-1,1]$.

Assumption (GM) was proved to hold for the discrete Anderson model in [CGK09]. In [K111], it is proved that assumption (D) holds for the discrete Anderson model in dimension 1 at any two distinct energies, and, in any dimension, for energies sufficiently far apart from each other.

Under these assumptions, we have

Theorem 1.11. Assume (IAD), (W), (GM), (Loc), and (D) hold. Pick $E_{0} \in I$ and $E_{0}^{\prime} \in I$ such that $E_{0} \neq E_{0}^{\prime}$ and $(1.42)$ is satisfied at $E_{0}$ and $E_{0}^{\prime}$.

When $|\Lambda| \rightarrow+\infty$, the point processes $\Xi\left(E_{0}, \omega, \Lambda\right)$ and $\Xi\left(E_{0}^{\prime}, \omega, \Lambda\right)$, defined in (1.40), converge weakly respectively to two independent Poisson processes on $\mathbb{R}$ with intensity the Lebesgue measure. That is, for $U_{+} \subset \mathbb{R}$ and $U_{-} \subset \mathbb{R}$ compact intervals and $\left\{k_{+}, k_{-}\right\} \in \mathbb{N} \times \mathbb{N}$, one has

$$
\mathbb{P}\left(\left\{\omega ; \begin{array}{l}
\#\left\{j ; \xi_{j}\left(E_{0}, \omega, \Lambda\right) \in U_{+}\right\}=k_{+} \\
\#\left\{j ; \xi_{j}\left(E_{0}^{\prime}, \omega, \Lambda\right) \in U_{-}\right\}=k_{-}
\end{array}\right\}\right) \underset{\Lambda \rightarrow \mathbb{Z}^{d}}{\rightarrow}\left(\frac{\left|U_{+}\right|^{k_{+}}}{k_{+} !} \mathrm{e}^{-\left|U_{+}\right|}\right)\left(\frac{\left|U_{-}\right|^{k_{-}}}{k_{-} !} \mathrm{e}^{-\left|U_{-}\right|}\right) .
$$

Theorem 1.11 naturally leads to wonder how far the energies $E_{0}$ and $E_{0}^{\prime}$ need to be from each other with respect to the scaling used to renormalize the eigenvalues for such a result to still hold. We prove

Theorem 1.12. Assume (IAD), (W), (GM), (Loc). Pick $E_{0} \in I$ such that (1.42) is satisfied Assume moreover that the density of states $\nu$ is continuous at $E_{0}$.

Consider two sequences of energies, say $\left(E_{\Lambda}\right)_{\Lambda}$ and $\left(E_{\Lambda}^{\prime}\right)_{\Lambda}$ such that

(1) $E_{\Lambda} \underset{\Lambda \rightarrow \mathbb{Z}^{d}}{\rightarrow} E_{0}$ and $E_{\Lambda}^{\prime} \underset{\Lambda \rightarrow \mathbb{Z}^{d}}{\rightarrow} E_{0}$,

(2) $|\Lambda| \cdot\left|N\left(E_{\Lambda}\right)-N\left(E_{\Lambda}^{\prime}\right)\right| \underset{\Lambda \rightarrow \mathbb{Z}^{d}}{\rightarrow}+\infty$.

Then, the point processes $\Xi\left(E_{\Lambda}, \omega, \Lambda\right)$ and $\Xi\left(E_{\Lambda}^{\prime}, \omega, \Lambda\right)$, defined in (1.40), converge weakly respectively to two independent Poisson processes on $\mathbb{R}$ with intensity the Lebesgue measure.

Theorem 1.12 shows that, in the localized regime, eigenvalues that are separated by a distance that is asymptotically infinite when compared to the mean spacing between the eigenlevels, behave as independent random variables. There are no interactions except at very short distances.

Assumption (2) can clearly not be omitted in Theorem 1.12; it suffices to consider e.g. $E_{\Lambda}, E_{\Lambda}^{\prime}$ s.t. $N\left(E_{\Lambda}\right)=N\left(E_{\Lambda}^{\prime}\right)+a|\Lambda|^{-1}$ to see that the two limit random processes are obtained as a shift from one another. 
1.8. The joint (energy - localization center) statistics. Recall that $E_{1}(\omega, \Lambda) \leq E_{2}(\omega, \Lambda) \leq$ $\cdots \leq E_{N}(\omega, \Lambda)$ denote the eigenvalues of $H_{\omega}(\Lambda)$ ordered increasingly and repeated according to multiplicity. Recall Lemma 1.1 and Lemma 1.2: it states that, to an eigenvector associated to $E_{j}(\omega, \Lambda)$, we can associate a center of localization that we denote by $x_{j}(\omega, \Lambda)$.

1.8.1. Uniform Poisson convergence for the joint (energy,center)-distribution. We now place ourselves in the same setting as in section 1.7.1. We prove

Theorem 1.13. Assume (IAD), (W), (M) and (Loc) hold. Let $E_{0}$ be an energy in I such that (1.45) holds for some $\tilde{\rho} \in[0, \rho /(1+d \rho))$. Pick $\alpha \in\left(\alpha_{d, \rho, \tilde{\rho}}, 1\right)$. Then, there exists $\delta>0$ such that,

- for any sequences of intervals $I_{1}=I_{1}^{\Lambda}, \ldots, I_{p}=I_{p}^{\Lambda}$ in $|\Lambda|^{1-\alpha} \cdot[-1,1]$ satisfying (1.46),

- for any sequences of cubes $C_{1}=C_{1}^{\Lambda}, \ldots, C_{p}=C_{p}^{\Lambda}$ in $[-1 / 2,1 / 2]^{d}$,

one has, for any sequences of integers $k_{1}=k_{1}^{\Lambda}, \cdots, k_{p}=k_{p}^{\Lambda} \in \mathbb{N}^{p}$,

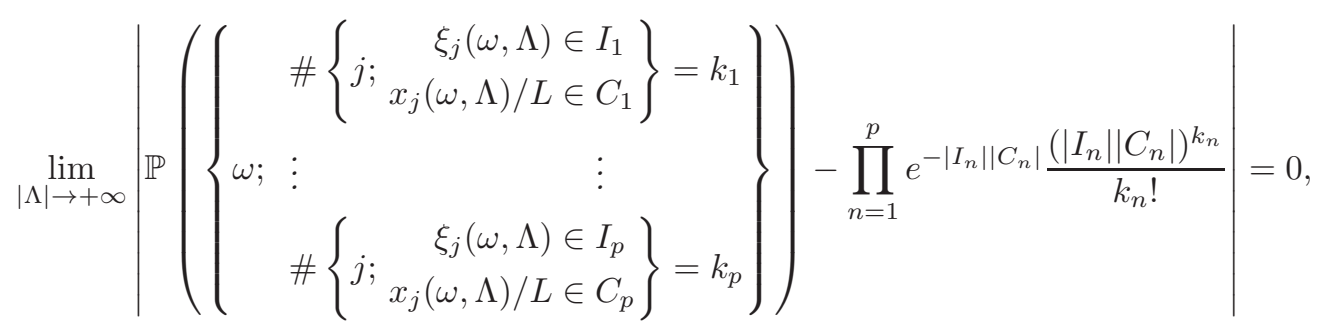

where the $\xi_{j}\left(\omega, \Lambda_{L}\right)$ 's are defined in (1.41).

In particular the point process defined as

$$
\Xi_{\Lambda}^{2}\left(\xi, x ; E_{0}, \Lambda\right)=\sum_{j=1}^{N} \delta_{\xi_{j}\left(\omega, \Lambda_{L}\right)}(\xi) \otimes \delta_{x_{j}(\omega, \Lambda) / L}(x)
$$

converges weakly to a Poisson point process on $\mathbb{R} \times \mathbb{R}^{d}$ with intensity the Lebesgue measure.

The joint (energy,center)-distribution given by $\Xi_{\Lambda}^{2}\left(\xi, x ; E_{0}, \Lambda\right)$ in (1.51) have been studied in [KN07], where they prove it converges weakly to a Poisson process.

We point out that in Theorem 1.13 intervals $I_{j}$ 's and cubes $C_{j}$ 's may depend on $\Lambda$. But the limit only depends on the product $\left|I_{j}\right|\left|C_{j}\right|$. We shall exploit this fact in the next result.

1.8.2. Covariant scaling joint (energy,center)-distribution. Fix $\xi \in(0,1)$ and an increasing sequence of scales $\ell=\left(\ell_{\Lambda}\right)_{\Lambda}$ such that

$$
\frac{\ell_{\Lambda}}{\log ^{1 / \xi}|\Lambda|} \underset{|\Lambda| \rightarrow+\infty}{\rightarrow}+\infty \quad \text { and } \quad \ell_{\Lambda} \leq|\Lambda|^{1 / d}
$$

Pick $E_{0} \in I$ so that $\nu\left(E_{0}\right)>0$. Consider the point process

$$
\Xi_{\Lambda}^{2}\left(\xi, x ; E_{0}, \ell\right)=\sum_{j=1}^{N} \delta_{\ell_{\Lambda}^{d}\left[N\left(E_{j}(\omega, \Lambda)\right)-N\left(E_{0}\right)\right]}(\xi) \otimes \delta_{x_{j}(\omega) / \ell_{\Lambda}}(x) .
$$

The process is valued in $\mathbb{R} \times \mathbb{R}^{d}$; actually, if $c \ell_{\Lambda} \geq|\Lambda|^{1 / d}$, it is valued in $\mathbb{R} \times(-c, c)^{d}$. Assume it exists and define the limit

$$
c_{\ell}=\lim _{|\Lambda| \rightarrow+\infty}|\Lambda|^{1 / d} \ell_{\Lambda}^{-1} \in[1,+\infty] .
$$

Note that if $\ell_{\Lambda}=L$, we recover (1.51). We prove 
Theorem 1.14. Assume (IAD), (W), (M) and (Loc) hold. Let $E_{0}$ be an energy in $I$ such that $(1.42)$ holds for some $\tilde{\rho} \in[0, \rho /(1+d \rho))$. The point process $\Xi_{\Lambda}^{2}\left(\xi, x ; E_{0}, \ell\right)$ converges weakly to a Poisson process on $\mathbb{R} \times\left(-c_{\ell}, c_{\ell}\right)^{d}$ with intensity measure the Lebesgue measure.

As a result, we see that, once the energies and the localization centers are scaled covariantly, the convergence to a Poisson process is true at any scale that is essentially larger than the localization width. This covariant scaling is very natural; it is the one prescribed by the Heisenberg uncertainty principle: the more precision we require in the energy variable, the less we can afford in the space variable. In this respect, the energies behave like a homogeneous symbol of degree $d$. This is quite different from what one obtains in the case of the Laplace operator.

1.8.3. Non-covariant scaling joint (energy,center)-distribution. One can also study what happens when the energies and localization centers are not scaled covariantly. Consider two increasing sequences of scales, say $\ell=\left(\ell_{\Lambda}\right)_{\Lambda}$ and $\tilde{\ell}=\left(\tilde{\ell}_{\Lambda}\right)_{\Lambda}$. Pick $E_{0}$ an energy in $I$ such that (1.42) holds for some $\tilde{\rho} \in[0, \rho /(1+d \rho))$. Consider the point process

$$
\Xi_{\Lambda}^{2}\left(\xi, x ; E_{0}, \ell, \tilde{\ell}\right)=\sum_{j=1}^{N} \delta_{\ell_{\Lambda}^{d}\left[N\left(E_{j}(\omega, \Lambda)\right)-N\left(E_{0}\right)\right]}(\xi) \otimes \delta_{x_{j}(\omega) / \tilde{\ell}_{\Lambda}}(x) .
$$

Then, we prove

Theorem 1.15. Assume (IAD), (W), (M) and (Loc) hold. Let $E_{0}$ be an energy in $I$ such that (1.42) holds for some $\tilde{\rho} \in[0, \rho /(1+d \rho))$. Assume the sequences of increasing scales $\ell=\left(\ell_{\Lambda}\right)_{\Lambda}$ and $\tilde{\ell}=\left(\tilde{\ell}_{\Lambda}\right)_{\Lambda}$ satisfy $(1.52)$. Assume that

$$
\text { if } \ell^{\prime}=o(L) \text { then } \frac{\ell_{\Lambda_{L+\ell^{\prime}}}}{\ell_{\Lambda_{L}}} \underset{|\Lambda| \rightarrow+\infty}{\rightarrow} 1 \text { and } \frac{\tilde{\ell}_{\Lambda_{L+\ell^{\prime}}}}{\tilde{\ell}_{\Lambda_{L}}} \underset{|\Lambda| \rightarrow+\infty}{\rightarrow} 1 .
$$

Let $J$ and $C$ be bounded measurable sets respectively in $\mathbb{R}$ and $\left(-c_{\tilde{\ell}}, c_{\tilde{\ell}}\right)^{d} \subset \mathbb{R}^{d}$. One has

(1) if, for some $\delta>0$, one has $\frac{\tilde{\ell}_{\Lambda}}{\ell_{\Lambda}} \leq|\Lambda|^{-\delta}$, then $\omega$ almost surely, for $\Lambda$ sufficiently large,

$$
\int_{J \times C} \Xi_{\Lambda}^{2}\left(\xi, x ; E_{0}, \ell, \tilde{\ell}\right) d \xi d x=0 .
$$

(2) if, for some $\delta>0$, one has $\frac{\tilde{\ell}_{\Lambda}}{\ell_{\Lambda}} \geq|\Lambda|^{\delta}$, then $\omega$ almost surely,

$$
\left(\frac{\ell_{\Lambda}}{\tilde{\ell}_{\Lambda}}\right)^{-d} \int_{J \times C} \Xi_{\Lambda}^{2}\left(\xi, x ; E_{0}, \ell, \tilde{\ell}\right) d \xi d x \underset{|\Lambda| \rightarrow+\infty}{\rightarrow}|J| \cdot|C| .
$$

Theorem 1.15 proves that the local energy levels and the localization centers become uniformly distributed in large energy windows if one conditions the localization centers to a much larger window. On the other hand, for a typical sample, if one looks for eigenvalues in an energy interval much smaller than the correctly scaled one with localization center in a cube, then asymptotically there are none.

If one replaces the polynomial growth or decay conditions of the ratio of scales $\ell_{\Lambda} / \tilde{\ell}_{\Lambda}$ by the condition that they tend to 0 or $\infty$, or if one omits condition (1.56), the results stays valid except for the fact that the convergence is not almost sure anymore but simply holds in probability; actually, one has convergence in some $L^{p}$ norm (see Remark 4.2 in section 4.2.3). 
1.9. Outline of the article. To complete this section, let us now briefly describe the architecture of the remaining parts of the paper that consist in the proofs of all the results stated in sections 0 and 1.

We start in section 2 with the computation of two important quantities related to our approximation scheme. Consider a cube $\Lambda$ and an energy interval $I$ such that $|I| \cdot|\Lambda|$ is small. In section 2 , we compute

- the probability that $H_{\omega}(\Lambda)$ has exactly any eigenvalue in $I$,

- the distribution of this eigenvalue conditioned on the fact that it is unique.

This distribution is used in the sequel to approximate the eigenvalue and localization center processes.

Section 3 is devoted to the proof of the approximation theorems, Theorems 1.1 and 1.2 , in section 3 Section 4 is devoted to the proof of the results on the spectral statistics. In section 5 , we derive the results for the full Hamiltonian i.e. we develop the other point of view presented in section 1.6. Finally, the appendix 6 is devoted to various technical results used in the course of the proofs, included a description of equivalent finite volume localization properties.

\section{The LOCAL Distribution of Eigenvalues}

In this section, we compute the distribution of unfolded eigenvalues.

2.1. The distribution of unfolded eigenvalues. Pick $1 \ll \ell^{\prime} \ll \ell$. Consider a cube $\Lambda$ of sidelength $\ell$ i.e. $\Lambda=\Lambda_{\ell}$ and an interval $I_{\Lambda}=\left[a_{\Lambda}, b_{\Lambda}\right] \subset I$ (i.e. $I_{\Lambda}$ is contained in the localization region). Consider the following random variables:

- $X=X\left(\Lambda, I_{\Lambda}\right)=X\left(\Lambda, I_{\Lambda}, \ell^{\prime}\right)$ is the Bernoulli random variable

$$
X=\mathbf{1}_{H_{\omega}(\Lambda) \text { has exactly one eigenvalue in } I_{\Lambda} \text { with localization center in } \Lambda_{\ell-\ell^{\prime}} ;} \text {; }
$$

- $\tilde{E}=\tilde{E}\left(\Lambda, I_{\Lambda}\right)$ is the eigenvalue of $H_{\omega}(\Lambda)$ in $I_{\Lambda}$ conditioned on $X=1$;

- $\tilde{\xi}=\tilde{\xi}\left(\Lambda, I_{\Lambda}\right)=\left(\tilde{E}\left(\Lambda, I_{\Lambda}\right)-a_{\Lambda}\right) /\left|I_{\Lambda}\right|$.

Clearly $\tilde{\xi}$ is valued in $[0,1]$; let $\tilde{\Xi}$ be its distribution function.

In the present section, we will describe the distribution of these random variables as $|\Lambda| \rightarrow+\infty$ and $\left|I_{\Lambda}\right| \rightarrow 0$. We prove

Lemma 2.1. Assume (W), (M) and (Loc) hold. For any $\nu \in(0,1)$, one has

$$
\left|\mathbb{P}(X=1)-N\left(I_{\Lambda}\right)\right| \Lambda \| \lesssim\left(|\Lambda|\left|I_{\Lambda}\right|\right)^{1+\rho}+N\left(I_{\Lambda}\right)|\Lambda| \ell^{\prime} \ell^{-1}+|\Lambda| e^{-\left(\ell^{\prime}\right)^{\nu}}
$$

where $N(E)$ denotes the integrated density of states of $H_{\omega}$.

One has, for all $x, y \in[0,1]$,

$$
|(\tilde{\Xi}(x)-\tilde{\Xi}(y)) \mathbb{P}(X=1)| \lesssim|x-y|\left|I_{\Lambda}\right||\Lambda| .
$$

Moreover, setting $N(x, y, \Lambda):=\left[N\left(a_{\Lambda}+x\left|I_{\Lambda}\right|\right)-N\left(a_{\Lambda}+y\left|I_{\Lambda}\right|\right)\right]|\Lambda|$, one has

$$
|(\tilde{\Xi}(x)-\tilde{\Xi}(y)) \mathbb{P}(X=1)-N(x, y, \Lambda)| \lesssim\left(|\Lambda|\left|I_{\Lambda}\right|\right)^{1+\rho}+|N(x, y, \Lambda)| \ell^{\prime} \ell^{-1}+|\Lambda| e^{-\left(\ell^{\prime}\right)^{\nu}} .
$$

This lemma differs from the usual computation of the DOS in the sense that the size of the interval decays as the thermodynamic limit is taken. A joint limit in the volume and the size of the interval has to be taken here. The price we pay for this joint limit is that we shall restrict ourselves to the localization regime, while the IDS exists in a broader region.

First let us note that, when we will use Lemma 2.1 in conjunction with Theorems 1.1 or 1.2, the role of $\Lambda$ will be played by the cube $\Lambda_{\ell}$.

Of course, estimates (2.1) and (2.3) are of interest mainly if their right hand side which is to be 
understood as an error is smaller than the main term. In (2.1), the main restriction comes from the requirement that $N\left(I_{\Lambda}\right)|\Lambda| \gg\left(|\Lambda|\left|I_{\Lambda}\right|\right)^{1+\rho}$ which is essentially a requirement that $N\left(I_{\Lambda}\right)$ should not be too small with respect to $\left|I_{\Lambda}\right|$ (similar to that found in Theorems 1.1 and 1.2). Lemma 2.1 will be used in conjunction with Theorems 1.1 and 1.2. The cube $\Lambda$ in Lemma 2.1 will be the cube $\Lambda_{\ell}$ in Theorems 1.1 and 1.2. Therefore, the requirements induced by the other two terms are less restrictive.

In (2.3), the main restriction comes from the requirement that $N(x, y, \Lambda) \gg\left(|\Lambda|\left|I_{\Lambda}\right|\right)^{1+\rho}$. This is essentially a requirement on the size of $|x-y|$. It should not be too small. On the other hand, we expect the spacing between the eigenvalues of $H_{\omega}\left(\Lambda_{L}\right)$ to be of size $\left|\Lambda_{L}\right|^{-1}$ (we keep the notations of Theorem 1.2 and recall that the cube $\Lambda$ in Lemma 2.1 will be the cube $\Lambda_{\ell}$ in Theorem 1.2). So to distinguish between the eigenvalues, one needs to be able to know $\tilde{\Xi}$ up to resolution $|x-y|\left|I_{\Lambda}\right| \asymp\left|\Lambda_{L}\right|^{-1}$. This will force us to use Lemma 2.1 on intervals $I_{\Lambda}$ such that $N\left(I_{\Lambda}\right) \asymp|\Lambda|^{-\alpha}$ for some $\alpha \in(0,1)$ close to 1 (see the discussion following Theorem 1.2 and section 4.3.1).

To prove Lemma 2.1, we will use

Lemma 2.2. Assume (W) and (Loc) hold in I a compact interval. For $\nu \in(0,1)$ and $1 \ll \ell^{\prime} \ll \ell$, let $N\left(J, \ell, \ell^{\prime}\right)$ be the number of eigenvalues of $H_{\omega}\left(\Lambda_{\ell}\right)$ in $J$ with localization center in $\Lambda_{\ell-\ell^{\prime}}$. Then, there exists $C>0$ such that, for $J \subset I$ an interval such that $|J| \geq e^{-\left(\ell^{\prime}\right)^{\nu}}$, one has

$$
\left|\mathbb{E}\left(N\left(J, \ell, \ell^{\prime}\right)\right)-N(J)\right| \Lambda_{\ell}|| \lesssim N(J)\left|\Lambda_{\ell}\right| \ell^{\prime} \ell^{-1}+\ell^{d} e^{-\left(\ell^{\prime}\right)^{\nu}}
$$

We turn to the proofs of Lemma 2.2 and 2.1

Proof of Lemma 2.2. Recall Lemma 1.1 and let $\mathcal{V}_{\Lambda_{\ell}}$ be the set of configurations given in Part (II) of Lemma 1.1 for some $\nu \in(0,1)$ given. Outside $\mathcal{V}_{\Lambda_{\ell}}$, we bound the number of eigenvalues of $H_{\omega}(\Lambda)$ by $C\left|\Lambda_{\ell}\right|$. Thus, one has

$$
\mathbb{E}\left(\mathbf{1}_{\omega \notin \mathcal{V}_{\Lambda_{\ell}}} N\left(J, \ell, \ell^{\prime}\right)\right) \lesssim \ell^{d} e^{-\ell^{\nu}} .
$$

Assume now that $\omega \in \mathcal{V}_{\Lambda_{\ell}}$. It follows from Lemma 3.1 that for such $\omega$ 's, one has

$$
\left.\operatorname{tr}\left(\mathbf{1}_{\Lambda_{\ell-2 \ell^{\prime}}} \mathbf{1}_{J_{-}}\left(H_{\omega}\right)\right)+O\left(\ell^{d} e^{-\left(\ell^{\prime}\right)^{\nu}}\right) \leq N\left(J, \ell, \ell^{\prime}\right)\right) \leq \operatorname{tr}\left(\mathbf{1}_{\Lambda_{\ell}} \mathbf{1}_{J_{+}}\left(H_{\omega}\right)\right)+O\left(\ell^{d} e^{-\left(\ell^{\prime}\right)^{\nu}}\right)
$$

where, for some $C>0, J_{+}=J+C e^{-\left(\ell^{\prime}\right)^{\nu}}[-1,1]$ and $J_{-}=J \backslash\left[(\mathbb{R} \backslash J)+C e^{-\left(\ell^{\prime}\right)^{\nu}}[-1,1]\right]$. Note that $|J|-2 C e^{-\left(\ell^{\prime}\right)^{\nu}} \leq\left|J_{-}\right| \leq\left|J_{+}\right| \leq|J|+2 C e^{-\left(\ell^{\prime}\right)^{\nu}}$.

Taking the expectation of the right hand side of (2.6), using the covariance for the operator $H_{\omega}$ and the Wegner estimate (W), we compute

$$
\mathbb{E}\left(\operatorname{tr}\left(\mathbf{1}_{\Lambda_{\ell}} \mathbf{1}_{J_{+}}\left(H_{\omega}\right)\right)\right)=N\left(J_{+}\right)\left|\Lambda_{\ell}\right|=N(J)\left|\Lambda_{\ell}\right|+O\left(\ell^{d} e^{-\left(\ell^{\prime}\right)^{\nu}}\right) .
$$

The left hand side is estimated in the same way. Plugging this back into the expectation of (2.6) and using (2.5), $\left|\Lambda_{\ell-2 \ell^{\prime}}\right|=\left|\Lambda_{\ell}\right|\left(1+C \ell^{\prime} \ell^{-1}\right)$, and the assumption that $|J| \geq e^{-\left(\ell^{\prime}\right)^{\nu}}$ easily yields $(2.4)$. This completes the proof of Lemma 2.2 .

Proof of Lemma 2.1. Using the notations of Lemma 2.2, note that $\mathbb{P}(X=1)=\mathbb{P}\left\{N\left(I_{\Lambda}, \ell, \ell^{\prime}\right)=1\right\}$. First, we relate $\mathbb{P}\left\{N\left(I_{\Lambda}, \ell, \ell^{\prime}\right)=1\right\}$ to $\mathbb{E}\left[N\left(J, \ell, \ell^{\prime}\right)\right]$. To do so, we follow the ideas used [Kri08, CGK09] to estimate the probability for $H_{\omega}(\Lambda)$ to have an eigenvalue in $J$. We notice that, as 
$N\left(J, \ell, \ell^{\prime}\right)$ is integer valued

$$
\begin{aligned}
\mathbb{E}\left[N\left(J, \ell, \ell^{\prime}\right)\right]-\mathbb{P}(X=1) & =\mathbb{E}\left[N\left(J, \ell, \ell^{\prime}\right)\right]-\mathbb{P}\left\{N\left(I_{\Lambda}, \ell, \ell^{\prime}\right)=1\right\} \\
& =\sum_{k=2}^{\infty} k \mathbb{P}\left\{N\left(I_{\Lambda}, \ell, \ell^{\prime}\right)=k\right\} \\
& \leq \sum_{k=2}^{\infty} k(k-1) \mathbb{P}\left\{\operatorname{tr} \mathbf{1}_{I_{\Lambda}}\left(H_{\omega}(\Lambda)\right)=k\right\} \\
& =\mathbb{E}\left\{\operatorname{tr} \mathbf{1}_{I_{\Lambda}}\left(H_{\omega}(\Lambda)\right)\left(\operatorname{tr} \mathbf{1}_{I_{\Lambda}}\left(H_{\omega}(\Lambda)\right)-1\right)\right\} .
\end{aligned}
$$

Thus, by our assumption (M), we know

$$
0 \leq \mathbb{E}\left[N\left(J, \ell, \ell^{\prime}\right)\right]-\mathbb{P}(X=1) \leq C|\Lambda|^{1+\rho}\left|I_{\Lambda}\right|^{1+\rho}
$$

The evaluation of $\mathbb{E}\left[N\left(J, \ell, \ell^{\prime}\right)\right]$ is then given by Lemma 2.2. This yields $(2.1)$.

The estimate (2.2) is an immediate consequence of the Wegner estimate (W) and the normalization of $\tilde{\Xi}$.

Set $I_{x, y, \Lambda}=\left[a_{\Lambda}+x\left|I_{\Lambda}\right|, a_{\Lambda}+y\left|I_{\Lambda}\right|\right]$. To prove (2.3), we write

$$
\begin{aligned}
\left|\mathbb{P}\left\{N\left(I_{x, y, \Lambda}, \ell, \ell^{\prime}\right)=1\right\}-(\tilde{\Xi}(x)-\tilde{\Xi}(y)) \mathbb{P}(X=1)\right| & \leq \mathbb{P}\left\{\begin{array}{l}
H_{\omega}(\Lambda) \text { has at least } \\
2 \text { eigenvalues in } I_{\Lambda}
\end{array}\right\} \\
& \lesssim\left(\left|\Lambda_{\ell}\right|\left|I_{\Lambda}\right|\right)^{1+\rho}
\end{aligned}
$$

using (M).

Replacing $I_{\Lambda}$ with the interval $I_{x, y, \Lambda}$ in the estimation of $\mathbb{P}\left(N\left(I_{\Lambda}, \ell, \ell^{\prime}\right)=1\right)$ yields the estimation of the probability $\mathbb{P}\left(N\left(I_{x, y, \Lambda}, \ell, \ell^{\prime}\right)=1\right)$ and, thus, completes the proof of (2.3).

The proof of Lemma 2.1 is complete.

Remark 2.1. The gist of Lemma 2.1 is that the local distribution of $\tilde{E}$ is that of the density of states i.e., in (2.3), the remainder terms should be negligible with respect to $N(x, y, \Lambda)$. Clearly, this will only be the case if $N(x, y, \Lambda) \gg\left(|\Lambda|\left|I_{\Lambda}\right|\right)^{1+\rho}$. This imposes a condition on the size of $|y-x|$ i.e. $y-x$ can't be too small. This restriction will be made clear in the following result.

If one uses the improved Minami estimates of [CGK09] in (2.9), one can improve

$$
\begin{aligned}
\mid(\tilde{\Xi}(x)-\tilde{\Xi}(y)) & \mathbb{P}(X=1)-N(x, y, \Lambda) \mid \\
& \leq C\left(|x-y|^{1+\rho}|\Lambda|^{1+\rho}\left|I_{\Lambda}\right|^{1+\rho}+|\Lambda|^{2}\left|I_{\Lambda}\right|\left|I_{x, y, \Lambda}\right|+N(x, y, \Lambda) \ell^{\prime} \ell^{-1}+|\Lambda| e^{-\left(\ell^{\prime}\right)^{\nu}}\right)
\end{aligned}
$$

and thus take advantage of the possible smallness of $I_{x, y, \Lambda}$ compared to $I_{\Lambda}$. So this will lift the above restriction, at least if for $J \subset I_{\Lambda}$, one has $N(J) \gtrsim|J|$. This can be done in some cases [GK112].

We now describe the distribution of the unfolded eigenvalues. Therefore we slightly change our notations to localize the quantities near some energy $E_{0}$. Let $1 \ll \ell^{\prime} \ll \ell$. Pick $E_{0} \in I$ such that (1.42) be satisfied and $I_{\Lambda}=\left[a_{\Lambda}, b_{\Lambda}\right]$. Recall that

- $X=X\left(\Lambda, E_{0}+I_{\Lambda}\right)=X\left(\Lambda, E_{0}+I_{\Lambda}, \ell^{\prime}\right)$ is the Bernoulli random variable

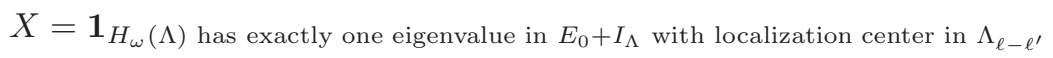

- $\tilde{E}=\tilde{E}\left(\Lambda, E_{0}+I_{\Lambda}\right)$ is this eigenvalue conditioned on $X=1$.

Define

$$
\xi=\frac{N(\tilde{E})-N\left(E_{0}+a_{\Lambda}\right)}{N\left(E_{0}+b_{\Lambda}\right)-N\left(E_{0}+a_{\Lambda}\right)}=\frac{N(\tilde{E})-N\left(E_{0}+a_{\Lambda}\right)}{N\left(E_{0}+I_{\Lambda}\right)} .
$$

The random variable $\xi$ is valued in $[0,1]$. Let $\Xi$ be its distribution function. We prove 
Lemma 2.3. Assume (W), (M) and (Loc) hold. Pick $E_{0}$ such that $(1.25)$ holds for $\rho^{\prime} \in(0, \rho)$. Fix $\nu \in(0,1)$. Assume, moreover, that

$$
e^{-\left(\ell^{\prime}\right)^{\nu}} \leq N\left(E_{0}+I_{\Lambda}\right)=o\left(|\Lambda|^{-\rho\left(1+\rho^{\prime}\right) /\left(\rho-\rho^{\prime}\right)}\right) \text { when }|\Lambda| \rightarrow+\infty .
$$

Then, for $1 \ll \ell^{\prime} \ll \ell$ and $(x, y) \in[0,1]^{2}$ such that $|x-y| \gg N\left(I_{\Lambda}\right)^{\left(\rho-\rho^{\prime}\right) /\left(1+\rho^{\prime}\right)}|\Lambda|^{\rho}$, one has

$$
\Xi(x)-\Xi(y)=(x-y)\left(1+O\left(\ell^{\prime} \ell^{-1}+e^{-\left(\ell^{\prime}\right)^{\nu}}+|x-y|^{-1} N\left(E_{0}+I_{\Lambda}\right)^{\frac{\rho-\rho^{\prime}}{1+\rho^{\prime}}}|\Lambda|^{\rho}\right)\right) .
$$

Recalling the discussion following Lemma 2.1, to be able to perform our analysis of the level spacings, we will need (2.12) to give a good approximation of $\Xi(x)-\Xi(y)$ for $|x-y| \ll\left(N\left(E_{0}+\right.\right.$ $\left.\left.I_{\Lambda}\right)\left|\Lambda_{L}\right|\right)^{-1}$ (recall that $\Lambda$ in Lemma 2.3 is $\Lambda_{\ell}$ in Theorem 1.2). Indeed, by Lemma 2.1 and Theorem 1.2, the number of eigenvalues of $H_{\omega}\left(\Lambda_{L}\right)$ in $I_{\Lambda}$ is asymptotic to $N\left(E_{0}+I_{\Lambda}\right)\left|\Lambda_{L}\right|$ (see Theorem 1.3).

Proof. Recall that the IDS $N$ is monotone by definition and Lipschitz continuous thanks to (W). Assumption (1.25) and the Wegner estimate (W) guarantee that, for $\Lambda$ sufficiently large, for $[a, b] \subset$ $E_{0}+I_{\Lambda}$, one has

$$
\frac{1}{C}(b-a) \leq\left|N^{-1}([a, b])\right| \leq(b-a)^{1 /\left(1+\rho^{\prime}\right)} .
$$

Here, $\left|N^{-1}([a, b])\right|$ denotes the Lebesgue measure of the interval $N^{-1}([a, b])$.

By the definitions of $\tilde{\xi}$ and $\xi$ (see the beginning of section 2.1 and (2.10)), for $x \in[0,1]$, one has

$$
\Xi(x)=\tilde{\Xi}\left[N^{-1}\left(N\left(E_{0}+a_{\Lambda}\right)+x N\left(E_{0}+\left|I_{\Lambda}\right|\right)\right)\right] .
$$

By Lemma 2.1 applied e.g. for $\nu$ replaced with $(1+\nu) / 2$, for $(x, y)$ as in $(2.12)$, one has

$$
\Xi(x)-\Xi(y)=(x-y) \frac{1+A+B+C}{1+A^{\prime}+B^{\prime}+C^{\prime}}
$$

where, using (2.13), the assumption on $|x-y|$ in (2.12) and the left hand side of (2.11), we compute

$$
\begin{aligned}
|A| & \lesssim \frac{\left(\left|I_{\Lambda}\right||\Lambda|\right)^{1+\rho}}{|x-y| N\left(I_{\Lambda}\right)|\Lambda|} \lesssim \frac{N\left(E_{0}+I_{\Lambda}\right)^{\left(\rho-\rho^{\prime}\right) /\left(1+\rho^{\prime}\right)}|\Lambda|^{\rho}}{|x-y|}, \quad|B| \lesssim \ell^{\prime} \ell^{-1}, \\
|C| & \lesssim \frac{e^{-\left(\ell^{\prime}\right)^{(1+\nu) / 2}}}{N\left(E_{0}+I_{\Lambda}\right)|x-y|} \lesssim e^{-\left(\ell^{\prime}\right)^{\nu}} .
\end{aligned}
$$

The quantities $\left|A^{\prime}\right|,\left|B^{\prime}\right|$ and $\left|C^{\prime}\right|$ are respectively bounded by the same bounds as $|A|,|B|$ and $|C|$ for $(x, y)=(0,1)$. This completes the proof of Lemma 2.3.

2.2. The proof of Theorem 1.3. Theorem 1.3 will be a consequence of Theorem 1.2 and Lemma 2.1. We use the notation of Theorem 1.2. Recall that the number of eigenvalues of $H_{\omega}(\Lambda)$ in $I_{\Lambda}$ is denoted by $N\left(I_{\Lambda}, \Lambda, \omega\right)$. The control of $N\left(I_{\Lambda}, \Lambda, \omega\right)$ will be useful in order obtain the level spacings statistics.

Recall the assumptions of Theorem 1.3:

- $N\left(I_{\Lambda}\right)(\log |\Lambda|)^{1 / \delta} \rightarrow 0$ as $|\Lambda| \rightarrow+\infty$

- $N\left(I_{\Lambda}\right)|\Lambda|^{1-\nu} \rightarrow+\infty$ as $|\Lambda| \rightarrow+\infty$

- $N\left(I_{\Lambda}\right)\left|I_{\Lambda}\right|^{-1-\tilde{\rho}} \rightarrow+\infty$ as $|\Lambda| \rightarrow+\infty$,

For $\delta>0$ sufficiently small, this guarantees that one can pick $\alpha>1$ large and $\ell=\ell_{\Lambda} \asymp(\log |\Lambda|)^{\alpha}$ and $\ell^{\prime}=\ell_{\Lambda}^{\prime} \asymp(\log |\Lambda|)^{1 / \xi}$ so that they fulfill all the assumptions of Theorem 1.2, in particular, (1.13), (1.14) and (1.17) for some $\xi \in(0,1)$ (see also the discussion following Theorem 1.2). 
The estimate (1.15) gives the probability of $\mathcal{Z}_{\Lambda}$, the set of configurations where one has a good description of most the eigenvalues. Moreover, for $\delta>0$ sufficiently small, the number of eigenvalues of $H_{\omega}(\Lambda)$ in $I_{\Lambda}$ that are not described by Theorem 1.2 is bounded by

$$
C N\left(I_{\Lambda}\right)|\Lambda|\left(N\left(I_{\Lambda}\right)^{\frac{\rho-\tilde{\rho}}{1+\rho}} \ell_{\Lambda}^{d(1+\rho)}+\left(\ell_{\Lambda}^{\prime}\right)^{d+1} \ell_{\Lambda}^{-1}\right) \leq C N\left(I_{\Lambda}\right)|\Lambda|(\log |\Lambda|)^{-\delta} .
$$

Consider the boxes $\left(\Lambda_{\ell}\left(\gamma_{j}\right)\right)_{1 \leq j \leq \tilde{N}}$ given by Theorem 1.2. Their number, say, $\tilde{N}$ satisfies $\tilde{N}=$ $|\Lambda| /\left|\Lambda_{\ell}\right|(1+o(1))$.

For $1 \leq j \leq \tilde{N}$, let $X_{j}=X\left(\Lambda_{\ell}\left(\gamma_{j}\right), I_{\Lambda}\right)$ i.e. $X_{j}$ is the Bernoulli random variable equal to 1 if $H_{\omega}\left(\Lambda_{\ell}\left(\gamma_{j}\right)\right)$ has exactly one eigenvalue in $I_{\Lambda}$ with localization center at a distance at least $\ell^{\prime}$ from $\partial \Lambda$ (see Theorem 1.2) and zero otherwise. It follows from Lemma 2.1 and the choice for $\left(\ell, \ell^{\prime}\right)$ in Theorem 1.2 made above that

$$
\left.\mathbb{P}\left(X_{j}=1\right)=N\left(I_{\Lambda}\right)\left|\Lambda_{\ell}\right|\left[1+o\left((\log |\Lambda|)^{-\delta}\right)\right)\right] .
$$

We have

$$
\left|N\left(I_{\Lambda}, \Lambda, \omega\right)-N\left(I_{\Lambda}\right)\right| \Lambda|| \leq\left|N\left(I_{\Lambda}, \Lambda, \omega\right)-\sum_{j=1}^{\tilde{N}} X_{j}\right|+\left|\sum_{j=1}^{\tilde{N}} X_{j}-N\left(I_{\Lambda}\right)\right| \Lambda||
$$

By (2.16), for $\omega \in \mathcal{Z}_{\Lambda}$, we have

$$
\left|N\left(I_{\Lambda}, \Lambda, \omega\right)-\sum_{j} X_{j}\right| \lesssim N\left(I_{\Lambda}\right)|\Lambda|(\log |\Lambda|)^{-\delta} .
$$

The second term in the right hand side of (2.18) is then bounded by a standard large deviation estimate for i.i.d. Bernoulli variables valued in $\{0,1\}$ with expectation $p=p(\tilde{N})$ s.t. $p \in(0,1 / 2]$ and $p \tilde{N} \sim N\left(I_{\Lambda}\right)|\Lambda| \rightarrow+\infty$ (see e.g. [Dur96]); for $\delta^{\prime} \in(0,1 / 2)$, it yields, for $|\Lambda|$ sufficiently large,

$$
\mathbb{P}\left(\left|\sum_{j=1}^{\tilde{N}} X_{j}-p \tilde{N}\right| \geq(p \tilde{N})^{\delta^{\prime}}\right) \leq e^{-(p \tilde{N})^{2 \delta^{\prime}-1} / 4} .
$$

Theorem 1.3 follows taking $\delta^{\prime}$ close to $1 / 2$, using (2.17) and noting that $p \tilde{N} \gg \log |\Lambda|$ by our assumptions on $N\left(I_{\Lambda}\right)$.

Remark 2.2. We can get a more precise version of Theorem 1.3 by optimizing in the intermediate scale $\ell$, the number of eigenvalues we miss in the picture of Theorem 1.2, namely by choosing $\ell$ so that $K n=K^{\prime} n^{\prime}$. Estimates can even be improved by resorting to higher order Minami estimates in order to bound the missing eigenvalues (replacing the crude deterministic bound given by the Weyl formula).

Remark 2.3. If $N(I) \ll|I|^{1+\rho}$, Theorem 1.3 still holds if one can improve on the Minami estimate, replacing one power of the interval length $|I|$ by $N(I)$. This can be done in some cases [GK112].

\section{The Proofs of Theorems 1.2 And 1.1}

Recall $\Lambda_{L}$ is a cube of side length $L$. Let $I_{\Lambda}$ be an interval inside $I$ the region of localization. We first prove the useful

Lemma 3.1. Assume (IAD), (W), (M) and (Loc). Consider scales $\ell^{\prime}, \ell$ so that $\left(\log \left|\Lambda_{L}\right|\right)^{1 / \xi} \ll$ $\ell^{\prime} \ll \ell \ll L$, and, for some given $\gamma \in \Lambda_{L}$, consider a box $\Lambda_{\ell}(\gamma)$ such that $\Lambda_{\ell-\ell^{\prime}}(\gamma) \subset \Lambda_{L}$. Let $\mathcal{W}_{\Lambda_{L}}$ be either the set $\mathcal{U}_{\Lambda_{L}}$ or $\mathcal{V}_{\Lambda_{L}}$ defined in Lemma 1.1. For L large enough, we have: 
(1) for any $\omega \in \mathcal{W}_{\Lambda_{L}}$, if $E(\omega)$ is an eigenvalue of $H_{\omega}\left(\Lambda_{L}\right)$ with a localized eigenfunction in the sense of (1.7) with a center of localization in $\Lambda_{\ell-\ell^{\prime}}(\gamma)$, then $H_{\omega}\left(\Lambda_{L} \cap \Lambda_{\ell}(\gamma)\right)$ has an eigenvalue in a neighborhood of $E(\omega)$ of size $\mathcal{O}\left(\mathrm{e}^{-\left(\ell^{\prime}\right)^{\xi} / 2}\right)$; moreover, if $\omega \in \mathcal{W}_{\Lambda_{\ell}(\gamma)}$, the corresponding eigenfunction is localized in the sense of (1.7).

(2) Assume now additionally that $\Lambda_{\ell}(\gamma) \subset \Lambda_{L}$. Then, conversely, for any $\omega \in \mathcal{W}_{\Lambda_{\ell}(\gamma)}$, if $E(\omega)$ is eigenvalue of $H_{\omega}\left(\Lambda_{L}\right)$ in $H_{\omega}\left(\Lambda_{\ell}(\gamma)\right)$ with an eigenfunction exponentially localized, in the sense of (1.7) with a center of localization in $\Lambda_{\ell-\ell^{\prime}}(\gamma)$, then $H_{\omega}\left(\Lambda_{L}\right)$ has an eigenvalue in a neighborhood of $E(\omega)$ of size $\mathcal{O}\left(\mathrm{e}^{-\left(\ell^{\prime}\right)^{\xi} / 2}\right)$; moreover, if $\omega \in \mathcal{W}_{\Lambda_{L}}$, the corresponding eigenfunction is localized in the sense of (1.7).

As a consequence of (1), (W) and (M), given an interval $I_{\Lambda}$,

- the probability to have at least one center of localization in $\Lambda_{\ell-\ell^{\prime}}(\gamma)$ corresponding to an eigenvalue of $H_{\omega}\left(\Lambda_{L}\right)$ in $I_{\Lambda}$ is bounded by $C\left(\mathbb{P}\left(\mathcal{W}_{\Lambda_{L}}^{c}\right)+\left|I_{\Lambda}\right| \ell^{d}+\ell^{d} e^{-\left(\ell^{\prime}\right)^{\xi} / 2}\right)$;

- the probability to have at least two centers of localization in $\Lambda_{\ell-\ell^{\prime}}(\gamma)$ corresponding to two eigenvalues of $H_{\omega}\left(\Lambda_{L}\right)$ in $I_{\Lambda}$ is bounded by $C\left(\mathbb{P}\left(\mathcal{W}_{\Lambda_{L}}^{c}\right)+\left(\left|I_{\Lambda}\right| \ell^{d}\right)^{(1+\rho)}+L^{d(1+\rho)} e^{-\rho\left(\ell^{\prime}\right)^{\xi} / 2}\right)$.

Similar results can be found in [K111].

Remark 3.1. In the first part of Lemma 3.1, we do not require the small cube $\Lambda_{\ell}(\gamma)$ to lie entirely inside the big cube $\Lambda_{L}$. This will be used in our analysis to treat the localization centers near the boundary of $\Lambda_{L}$.

If one has $\Lambda_{\ell}(\gamma) \subset \Lambda_{L}$, then, using Lemma 2.1, the bound on the probability to have at least one center of localization in $\Lambda_{\ell-\ell^{\prime}}(\gamma)$ corresponding to an eigenvalue of $H_{\omega}\left(\Lambda_{L}\right)$ in $I_{\Lambda}$ can be improved into $C\left(\mathbb{P}\left(\mathcal{W}_{\Lambda_{L}}^{c}\right)+N\left(I_{\Lambda}\right) \ell^{d}+\ell^{d} e^{-\left(\ell^{\prime}\right)^{\xi} / 2}\right)$.

Finally, we note that, in the last part of Lemma 3.1, it is of importance that the probability that appears, namely $\mathbb{P}\left(\mathcal{W}_{\Lambda_{L}}^{c}\right)$, is the one related to the box $\Lambda_{L}$, and not to a small box of size $\ell$.

Proof. We start with the point (1). Let $\varphi=\varphi_{\omega, \Lambda_{L}}$ be the eigenfunction associated to the center $x(\omega)$, and $E(\omega) \in I_{\Lambda}$ the corresponding eigenvalue. Let $\Psi_{\ell}$ be a smooth characteristic function covering the cube $\Lambda_{\ell}(\gamma)$, i.e. $\Psi_{\ell}$ such that $\operatorname{supp} \Psi_{\ell} \subset \Lambda_{\ell}(\gamma), \Psi_{\ell}=1$ on $\Lambda_{\ell-\frac{1}{2} \ell^{\prime}}(\gamma), \operatorname{supp} \nabla \Psi_{\ell} \subset$ $\Lambda_{\ell}(\gamma) \backslash \Lambda_{\ell-\frac{1}{2} \ell^{\prime}}(\gamma)$

Since $\omega \in \mathcal{U}_{\Lambda_{L}}$, we have $\left\|\Psi_{\ell} \varphi\right\| \geq \frac{1}{2}$ for $\ell^{\prime}$ large enough. Set $\eta_{\ell}:=\Psi_{\ell} \varphi /\left\|\Psi_{\ell} \varphi\right\|$. Then, $\eta_{\ell}$ is an approximate eigenvector of the Hamiltonian $H_{\omega}\left(\Lambda_{\ell}(\gamma)\right)$, in the sense that $\left\|\eta_{\ell}\right\|=1$ and

$$
\left\|\left(H_{\omega}\left(\Lambda_{\ell}(\gamma)\right)-E\right) \eta_{\ell}\right\| \leq 2\left\|\left[H_{\omega}\left(\Lambda_{\ell}(\gamma)\right), \Psi_{\ell}\right] \varphi\right\| \lesssim \sup _{\operatorname{supp} \nabla \Psi_{\ell}}|\varphi| \lesssim \mathrm{e}^{-\left(\ell^{\prime}\right)^{\xi}} .
$$

It follows that $H_{\omega}\left(\Lambda_{\ell}(\gamma)\right)$ has an eigenvalue in the interval $\left[E-c \mathrm{e}^{-\left(\ell^{\prime}\right)^{\xi}}, E+c \mathrm{e}^{-\left(\ell^{\prime}\right)^{\xi}}\right]$.

Let us prove point (2). Recall $\Psi_{\ell}$ above. Let $\varphi=\varphi_{\omega, \Lambda_{\ell}(\gamma)} \in \mathrm{L}^{2}\left(\Lambda_{\ell}(\gamma)\right)$ be the eigenfunction associated to $x(\omega)$. Set $\eta_{\ell}=\Psi_{\ell} \varphi$ on $\Lambda_{\ell}(\gamma)$ and $\eta_{\ell}=0$ on $\Lambda_{L} \backslash \Lambda_{\ell}(\gamma)$. Since $\left.\operatorname{dist}(x(\omega)), \partial \Lambda_{\ell}(\gamma)\right) \geq \ell^{\prime}$, it is immediate to see that $\eta_{\ell}$ is an approximate eigenfunction of $H_{\omega}\left(\Lambda_{L}\right)$ in the sense that

$$
\left\|\left(H_{\omega}\left(\Lambda_{L}\right)-E\right) \eta_{\ell}\right\| \lesssim \sup _{\operatorname{supp} \nabla \Psi_{\ell}}|\varphi| \lesssim \mathrm{e}^{-\left(\ell^{\prime}\right)^{\xi} / 2} .
$$

The first consequence is immediate. For the second, if the two eigenvalues of $H_{\omega}(\Lambda)$ are at a a distance at least $e^{-\left(\ell^{\prime}\right)^{\xi} / 2}$ from each other, by point (2), they give rise to two distinct eigenvalues of $H_{\omega}\left(\Lambda_{\ell}(\gamma)\right)$; thus, we can apply (M). If they are closer, we bound the probability using (M) for $H_{\omega}\left(\Lambda_{L}\right)$.

This completes the proof of Lemma 3.1.

We now turn to the proofs of Theorems 1.1 and 1.2. 
Proof of Theorem 1.1. For $\beta^{\prime}>0$ sufficiently small (this will be defined precisely below) and $\beta>\beta^{\prime}$ to be chosen later, set $\ell^{\prime}=L^{\beta^{\prime}}$ and $\ell$ so that $\left(\ell+\ell^{\prime}\right) k+\ell^{\prime}=L$, where $k=\left[L^{1-\beta}\right]$. Note that $\ell=\mathcal{O}\left(L^{\beta}\right)$ in the large volume limit. With such definitions we can pick equally distributed boxes of size $\ell$ in $\Lambda_{L}$ (with distance $\ell^{\prime}$ between two neighbors) satisfying the conditions of the theorem. Note that for $L$ large enough $\ell^{\prime}>R$, so that events based on distinct boxes $\Lambda_{\ell}\left(\gamma_{j}\right)$ 's are independent.

In this proof, we shall use the localization property described by Lemma 1.1 Part (I).

Up to a probability less than $C\left|\Lambda_{L}\right|^{(1-\beta)-\beta p} \lesssim\left|\Lambda_{L}\right|^{-1}$ provided $p \geq 2 \beta^{-1}-1$, we can assume that all the boxes $\Lambda_{\ell}(\gamma)$ satisfy Part (I) of Lemma 1.1, since $p$ in Lemma 1.1 can be chosen arbitrary large. Since $\alpha-\alpha_{d, \rho, \tilde{\rho}}<1$ we can neglect this probability.

Recall $N\left(I_{\Lambda}\right) \asymp\left|\Lambda_{L}\right|^{-\alpha}$. Since $N\left(I_{\Lambda}\right) \lesssim\left|I_{\Lambda}\right|$, we have $\mathbb{P}\left(\mathcal{U}_{\Lambda_{L}}^{c}\right) \lesssim\left|I_{\Lambda}\right| \ell^{d}$ and $\mathbb{P}\left(\mathcal{U}_{\Lambda_{L}}^{c}\right) \lesssim\left|I_{\Lambda}\right|^{1+\rho} \ell^{2 d}$, provided $p>0$ in Lemma 1.1 is large enough.

Let $\mathcal{S}_{\ell, L}$ be the set of boxes $\Lambda_{\ell-\ell^{\prime}}\left(\gamma_{j}\right) \subset \Lambda_{L}$ containing at least two centers of localization of $H_{\omega}\left(\Lambda_{L}\right)$. It follows from Lemma 3.1 and (1.42) that,

$$
\mathbb{P}\left(\# \mathcal{S}_{\ell, L} \geq 1\right) \lesssim\left|\Lambda_{L}\right|^{1-\beta}\left(\left|\Lambda_{L}\right|^{\beta}\left|I_{\Lambda}\right|\right)^{1+\rho} \leq\left|\Lambda_{L}\right|^{1+\beta \rho} N\left(I_{\Lambda}\right)^{\frac{1+\rho}{1+\rho}} \lesssim\left|\Lambda_{L}\right|^{1+\beta \rho-\alpha \frac{1+\rho}{1+\rho}} .
$$

To insure that all the centers of $H_{\omega}\left(\Lambda_{L}\right)$ fall inside one of the $\Lambda_{\ell}\left(\gamma_{j}\right)$ 's and actually sufficiently well inside (by a distance $\ell^{\prime}$ ), we define $\Upsilon \subset \Lambda_{L}$ as the set $\Lambda_{L} \backslash \cup_{j} \Lambda_{\ell}\left(\gamma_{j}\right)$ enlarged by a length $\ell^{\prime}$. In other terms $\Upsilon=\Lambda_{L} \backslash \cup_{j} \Lambda_{\ell-\ell^{\prime}}\left(\gamma_{j}\right)$. One has $|\Upsilon| \lesssim\left|\Lambda_{L}\right| \ell^{\prime} \mid \ell$. We consider a partition $\Upsilon=\bigcup_{m=1}^{2^{d-1}} \Upsilon_{m}$, with $\Upsilon_{m} \cap \Upsilon_{m^{\prime}}=\emptyset$ if $m \neq m^{\prime}$, each $\Upsilon_{m}$ being a union of boxes of side length $\ell^{\prime}$ which are two by two distant by at least $\ell^{\prime}$. For each given $m$, the distance between two boxes of $\Upsilon_{m}$ is larger than $\ell^{\prime}$, so that we can enlarge each box in $\Upsilon_{m}$ by, say, $\frac{1}{10} \ell^{\prime}$, except for sides of boxes that coincide with the boundary of $\Lambda_{L}$. It follows from Lemma 3.1 and the Wegner estimate that,

$$
\begin{aligned}
& \mathbb{P}\left(H_{\omega}\left(\Lambda_{L}\right) \text { has a center of localization in } \Upsilon\right) \\
& \lesssim \sum_{m} \mathbb{P}\left(H_{\omega}\left(\Lambda_{L}\right) \text { has a center of localization in } \Upsilon_{m}\right) \\
& \lesssim \sum_{m}\left|\Upsilon_{m}\right|\left|I_{\Lambda}\right| \lesssim|\Upsilon| N\left(I_{\Lambda}\right)^{\frac{1}{1+\rho}} \lesssim\left|\Lambda_{L}\right|^{1-\frac{1}{d}\left(\beta-\beta^{\prime}\right)-\frac{\alpha}{1+\rho}}
\end{aligned}
$$

We, thus, require that

$$
\alpha>(1+\tilde{\rho}) \max \left(\frac{1+\beta \rho}{1+\rho}, 1-\frac{1}{d}\left(\beta-\beta^{\prime}\right)\right) .
$$

Optimization yields

$$
\beta=\frac{d \rho+\beta^{\prime}(1+\rho)}{(d+1) \rho+1}, \quad \alpha>(1+\tilde{\rho})\left(1-\frac{d \rho+\beta^{\prime}(1+\rho)}{(d+1) \rho+1}\right) .
$$

We, thus, require $\alpha_{d, \rho, \tilde{\rho}}<\alpha$ where

$$
\alpha_{d, \rho, \tilde{\rho}}=(1+\tilde{\rho})\left(1-\frac{\rho}{\rho(d+1)+1}\right)=(1+\tilde{\rho}) \frac{\rho d+1}{\rho(d+1)+1}<1,
$$

which is our assumption (1.11).

It follows from (3.1) and (3.2) that with probability larger than $1-c\left|\Lambda_{L}\right|^{-\left(\alpha-\alpha_{d, \rho, \tilde{\rho})}\right.}$, item (2) of Theorem 1.1 holds, as well as the "only if" part of item (3).

Next, item (2) of Lemma 3.1 implies that item (1) as well as the "if" part of item (3) of Theorem 1.1 hold, with probability at least $1-c\left|\Lambda_{L}\right|^{-\alpha+\beta-\frac{1}{d}\left(\beta-\beta^{\prime}\right)} \geq 1-c\left|\Lambda_{L}\right|^{-\left(\alpha-\alpha_{d, \rho, \tilde{\rho})}\right.}$.

This completes the proof of Theorem 1.1. 
Proof of Theorem 1.2. For a scale $\ell_{\Lambda}$ given, we set $q=\left[\left(L-\ell^{\prime}\right) /\left(\ell_{\Lambda}+\ell^{\prime}\right)\right]$. Then we may adjust the scale $\ell_{\Lambda}$ by enlarging it to a new scale $\ell$ so that $\left(\ell+\ell^{\prime}\right) q+\ell^{\prime}=L$ and $0 \leq \ell-\ell_{\Lambda} \lesssim \ell_{\Lambda}^{2} /\left|\Lambda_{L}\right|=o\left(\ell_{\Lambda}\right)$. As a consequence, we can consider a collection of boxes $\Lambda_{\ell}\left(\gamma_{j}\right)$ equally distant to their closest neighbors by a length $\ell^{\prime}$ and satisfying the description of the theorem. In particular, events based on different boxes are independent.

In this proof, we shall use the localization property described by Lemma 1.1 Part (II).

For $L$ sufficiently large, up to a probability $\lesssim\left|\Lambda_{L}\right|^{(1-\beta)} \exp ^{-\ell^{\nu}} \leq\left|\Lambda_{L}\right|^{-q}$, with $q>0$ arbitrary large, we can assume that all the boxes $\Lambda_{\ell}(\gamma)$ satisfy Part (II) of Lemma 1.1.

It follows from (1.13) and Lemma 1.1 that for $\ell$ large enough, we have $\mathbb{P}\left(\mathcal{V}_{\Lambda_{L}}^{c}\right) \lesssim\left|I_{\Lambda}\right| \ell^{d}$ and $\mathbb{P}\left(\mathcal{V}_{\Lambda_{L}}^{c}\right) \lesssim\left|I_{\Lambda}\right|^{1+\rho} \ell^{2 d}$.

Let $\mathcal{S}_{\ell, L}$ be the set of disjoint boxes $\Lambda_{\ell}\left(\gamma_{j}\right) \subset \Lambda_{L}$ containing at least 2 centers of localization of $H_{\omega}\left(\Lambda_{L}\right)$. We set $n=\ell^{d}$. It follows from Lemma 3.1 (taking into account $\ell^{\prime} \ll \ell$ ) that, using independence and Stirling's formula,

$$
\begin{aligned}
\mathbb{P}\left(\sharp\left(\mathcal{S}_{\ell, L} \geq k\right)\right. & \lesssim\left(\begin{array}{c}
\left|\Lambda_{L}\right| / n \\
k
\end{array}\right)\left(\left|I_{\Lambda}\right| n\right)^{(1+\rho) k} \\
& \lesssim\left(\mathrm{e}\left|\Lambda_{L}\right| /(n k)\right)^{k}\left(\left|I_{\Lambda}\right| n\right)^{(1+\rho) k}=\left(\frac{\mathrm{e}\left|\Lambda_{L}\right|}{k} N\left(I_{\Lambda}\right)^{\frac{1+\rho}{1+\rho^{\prime}}} n^{\rho}\right)^{k} \lesssim 2^{-k},
\end{aligned}
$$

if we choose

$$
k \geq K:=\left[2 \mathrm{e} N\left(I_{\Lambda}\right)\left|\Lambda_{L}\right|\left(N\left(I_{\Lambda}\right)^{\frac{\rho-\rho^{\prime}}{1+\rho^{\prime}}} n^{\rho}\right)\right]+1 .
$$

Note that

$$
K \asymp \frac{\left|\Lambda_{L}\right|}{n}\left(N\left(I_{\Lambda}\right)^{\frac{1}{1+\rho}} n\right)^{1+\rho}=o\left(\frac{\left|\Lambda_{L}\right|}{n}\right)
$$

by assumption. As a consequence, we get that

$$
\mathbb{P}\left(\#\left(\mathcal{S}_{\ell, L}\right) \geq K\right) \lesssim 2^{-K} .
$$

So that, with probability larger than $1-2^{-K}$, we can assume that the boxes $\Lambda_{\ell}\left(\gamma_{j}\right)$, except at most $K$ of them, contain at most one center of localization.

We now control the number of centers of localization that may be contained in these $K$ exceptional boxes. In a box of size $\ell$, the deterministic a priori bound on the number of eigenvalues guarantees that this number is bounded by $\lesssim \ell^{d}:=n$ (see e.g. [RS78]). Using this crude estimate the number of eigenvalues we miss with these $K$ boxes is bounded by

$$
K n \lesssim N\left(I_{\Lambda}\right)\left|\Lambda_{L}\right|\left(N\left(I_{\Lambda}\right)^{\frac{\rho-\rho^{\prime}}{1+\rho^{\prime}}} n^{1+\rho}\right)=o\left(N\left(I_{\Lambda}\right)\left|\Lambda_{L}\right|\right),
$$

provided

$$
N\left(I_{\Lambda}\right)^{\frac{\rho-\rho^{\prime}}{1+\rho^{\prime}}} n^{1+\rho}=o(1) .
$$

We now turn to the complement of the $\Lambda_{\ell}\left(\gamma_{j}\right)$ 's, that is

$$
\Upsilon=\Lambda_{L} \backslash \bigcup_{j} \Lambda_{\ell-\ell^{\prime}}\left(\gamma_{j}\right)
$$

and we consider a partition of $\Upsilon$ in terms of $2^{d-1}$ sets of boxes of side length $\ell^{\prime}$. More precisely, $2^{d-1}$

$\Upsilon=\bigcup_{m=1} \Upsilon_{m}$, with $\Upsilon_{m} \cap \Upsilon_{m^{\prime}}=\emptyset$ if $m \neq m^{\prime}$, each $\Upsilon_{m}$ is a union of boxes of side length $\ell^{\prime}$ which are two by two distant by at least $\ell^{\prime}$.

Clearly, one has $|\Upsilon| \lesssim\left|\Lambda_{L}\right| \ell^{\prime} / \ell$.

Let $\mathcal{S}_{\ell, L}^{\prime}$ be the set of boxes $\Lambda_{\ell^{\prime}}\left(\gamma_{j}\right) \in \Upsilon$ containing at least one centers of localization of $H_{\omega}\left(\Lambda_{L}\right)$. 
From considerations similar to those above, for each given $m$, taking into account that boxes in $\Upsilon_{m}$ may be enlarged by, say, $\frac{1}{10} \ell^{\prime}$, for the distance between two of them is larger than $\ell^{\prime}$, it follows from Lemma 3.1 and Wegner estimate that, using independence and Stirling formula,

$$
\begin{aligned}
\mathbb{P}\left(\#\left(\mathcal{S}_{\ell, L}^{\prime} \cap \Upsilon_{m}\right) \geq K^{\prime}\right) & \lesssim \sum_{k \geq K^{\prime}}\left(\begin{array}{c}
\left|\Upsilon_{m}\right| /\left(\ell^{\prime}\right)^{d} \\
k
\end{array}\right)\left(C\left|I_{\Lambda}\right|\left(\ell^{\prime}\right)^{d}\right)^{k} \\
& \lesssim\left(\frac{C\left|\Lambda_{L}\right| N\left(I_{\Lambda}\right)^{\frac{1}{1+\rho^{\prime}}} \ell^{\prime}}{k \ell}\right)^{k} \lesssim 2^{-K^{\prime}}
\end{aligned}
$$

where $C$ is a constant that varies but only depends on the constant appearing in Wegner and $d$, and provided one sets, for $C^{\prime}>C$,

$$
K^{\prime}:=\left[\frac{C^{\prime}\left|\Lambda_{L}\right| N\left(I_{\Lambda}\right)^{\frac{1}{1+\rho^{\prime}}} \ell^{\prime}}{\ell}\right]+1 .
$$

Note that

$$
K^{\prime} \asymp \frac{\left|\Lambda_{L}\right|}{n}\left(N\left(I_{\Lambda}\right)^{\frac{1}{1+\rho^{\prime}}} n \frac{\ell^{\prime}}{\ell}\right)=o\left(\frac{\left|\Lambda_{L}\right|}{n}\right)
$$

by assumption. As a consequence, one computes

$$
\begin{aligned}
\mathbb{P}\left(\# \mathcal{S}_{\ell, L}^{\prime} \geq 2^{d-1} K^{\prime}\right) & \leq \mathbb{P}\left(\exists m, \#\left(\mathcal{S}_{\ell, L}^{\prime} \cap \Upsilon_{m}\right) \geq K^{\prime}\right) \\
& \leq \sum_{m=1}^{2^{d-1}} \mathbb{P}\left(\#\left(\mathcal{S}_{\ell, L}^{\prime} \cap \Upsilon_{m}^{\prime}\right) \geq K^{\prime}\right) \lesssim 2^{-K^{\prime}} .
\end{aligned}
$$

Hence, up to $2^{d-1} K^{\prime}$ boxes, we can assume that boxes of size $\ell^{\prime}$ in $\Upsilon^{\prime}$ contain at most one center of localization.

The maximal number of eigenvalues that can be contained in these $2^{d-1} K^{\prime}$ bad boxes is $\lesssim K^{\prime} n^{\prime}=$ $o\left(N\left(I_{\Lambda}\right)\left|\Lambda_{L}\right|\right)$ provided

$$
\ell \gg\left(\ell^{\prime}\right)^{d+1} \text {. }
$$

Combining (3.8) and (3.11), we see that the intermediate scale $\ell$ has to satisfy (1.17). To summarize, we proved that the picture described by Theorem 1.2 holds with a probability larger than

$$
\begin{aligned}
1-\mathrm{e}^{-c K}-\mathrm{e}^{-c K^{\prime}} \geq 1-\exp \left(-c N\left(I_{\Lambda}\right)\left|\Lambda_{L}\right|\left(N\left(I_{\Lambda}\right)^{\frac{\rho-\rho^{\prime}}{1+\rho^{\prime}}} \ell^{d \rho}\right)\right) & \\
& -\exp \left(-c N\left(I_{\Lambda}\right)\left|\Lambda_{L}\right|\left(N\left(I_{\Lambda}\right)^{-\frac{\rho^{\prime}}{1+\rho^{\prime}}} \ell^{\prime} \ell^{-1}\right)\right)
\end{aligned}
$$

Moreover, the number of eigenvalues of $H_{\omega}\left(\Lambda_{L}\right)$ that are not described by this picture is bounded by

$$
\begin{aligned}
C\left(K n+K^{\prime} n^{\prime}\right) & \lesssim N\left(I_{\Lambda}\right)\left|\Lambda_{L}\right|\left(\left(N\left(I_{\Lambda}\right)^{\frac{\rho-\rho^{\prime}}{1+\rho^{\prime}}} \ell^{d(1+\rho)}+N\left(I_{\Lambda}\right)^{-\frac{\rho^{\prime}}{1+\rho^{\prime}}} \frac{\left(\ell^{\prime}\right)^{d+1}}{\ell}\right)\right. \\
& =o\left(N\left(I_{\Lambda}\right)\left|\Lambda_{L}\right|\right),
\end{aligned}
$$

provided (1.17) holds.

This completes the proof of Theorem 1.2.

\section{The spectral statistics}

In this section, we prove most of the results on the local spectral statistics described in section 1. The whole of our analysis relies on Theorems 1.1 and 1.2. 
4.1. Convergence of the local levels statistics. We first prove the uniform Poisson convergence, Theorem 1.10, of which Theorem 1.9 is an immediate consequence if one takes into account Remark 4.1 to relax assumption (1.45) into assumption (1.42).

4.1.1. Proof of Theorem 1.10. We keep the notations of section 1.7.1. Recall that $\Lambda=\Lambda_{L}$. Under the assumptions of Theorem 1.10, we can apply Theorem 1.1 to the interval $I_{\Lambda}=N^{-1}\left(N\left(E_{0}\right)+\right.$ $\left.|\Lambda|^{-\alpha}[-1,1]\right)$. Let $\mathcal{Z}_{\Lambda}$ be the set of configurations where the conclusions of Theorem 1.1 for this interval hold and let $\tilde{N}$ be the number of the cubes constructed in Theorem 1.1 and $\left(\Lambda_{\ell}\left(\gamma_{j}\right)\right)_{1 \leq j \leq \tilde{N}}$ be those cubes. Then, $\tilde{N}=|\Lambda|^{1-\beta}\left(1-O\left(|\Lambda|^{-(1-\beta) / d}\right)\right)$.

Recall that $\xi_{j}\left(E_{0}, \omega, \Lambda\right)$ is defined by (1.41) and consider the event

$$
\Omega_{I_{1}, k_{1} ; I_{2}, k_{2} ; \cdots ; I_{p}, k_{p}}^{\Lambda}:=\bigcap_{l=1}^{p}\left\{\omega ; \#\left\{j ; \xi_{j}(\omega, \Lambda) \in I_{l}\right\}=k_{l}\right\} .
$$

Pick $\delta d<p:=\beta^{\prime} \xi$ where $\beta^{\prime}$ is given by Theorem 1.1 and $\delta$ by (1.46).

As $\mathbb{P}\left(\mathcal{Z}_{\Lambda}\right) \rightarrow 1$, to prove Theorem 1.10 , it suffices to prove that

$$
\mathbb{P}\left(\Omega_{I_{1}, k_{1} ; I_{2}, k_{2} ; \cdots ; I_{p}, k_{p}}^{\Lambda} \cap \mathcal{Z}_{\Lambda}\right)-e^{-\left|I_{1}\right|} \frac{\left|I_{1}\right|^{k_{1}}}{k_{1} !} \cdots e^{-\left|I_{p}\right|} \frac{\left|I_{p}\right|^{k_{p}}}{k_{p} !} \underset{|\Lambda| \rightarrow+\infty}{\rightarrow} 0 .
$$

Recall that the function $N$ is non decreasing and continuous; thus, for $I$ an interval, the set $N^{-1}(I)$ is an interval. For a cube $\Lambda$ and an interval $I$, define the Bernoulli random variable $X_{\Lambda_{\ell}, I}$ by

$$
X_{\Lambda_{\ell}, I}=\mathbf{1}_{H_{\omega}\left(\Lambda_{\ell}\right) \text { has an e.v. in } N^{-1}\left[N\left(E_{0}\right)+|\Lambda|^{-1} I\right] \text { with localization center in } \Lambda_{\ell-\ell^{\prime}}} .
$$

Here, the length scales $\ell$ and $\ell^{\prime}$ are taken as in Theorem 1.1 that is $\ell \asymp L^{\beta}$ and $\ell^{\prime} \asymp L^{\beta^{\prime}}$. Notice that, using the notations of section 2 , one has $X_{\Lambda_{\ell}, I}=X\left(\Lambda_{\ell}, N^{-1}\left[N\left(E_{0}\right)+|\Lambda|^{-1} I\right], \ell^{\prime}\right)$.

We are first going to eliminate a degenerate case i.e the case when the length of one interval $\left|I_{j}\right|$ goes to 0 . Assume $\left|I_{1}\right| \leq \varepsilon$ for some $\varepsilon$ small fixed. Let us first assume that $k_{1} \neq 0$. Then, by the description given by Theorem 1.1, for some $\eta>0$, for $L$ sufficiently large, one has

$$
\begin{aligned}
\mathbb{P}\left(\Omega_{I_{1}, k_{1} ; I_{2}, k_{2} ; \cdots ; I_{p}, k_{p}}^{\Lambda} \cap \mathcal{Z}_{\Lambda}\right) \leq & \mathbb{P}\left(\Omega_{I_{1}, k_{1}}^{\Lambda} \cap \mathcal{Z}_{\Lambda}\right) \lesssim \tilde{N} \mathbb{P}\left(X_{\Lambda_{\ell}(\gamma), I_{1}+e^{-L^{\eta}}[-1,1]}=1\right) \\
& \lesssim \tilde{N} \cdot N\left(N^{-1}\left[N\left(E_{0}\right)+|\Lambda|^{-1}\left(I_{1}+e^{-L^{\eta}}[-1,1]\right)\right]\right)\left|\Lambda_{\ell}\right| \\
& \quad+\tilde{N}\left(\left|\Lambda_{\ell}\right|\left|N^{-1}\left[N\left(E_{0}\right)+|\Lambda|^{-1}\left(I_{1}+e^{-L^{\eta}}[-1,1]\right)\right]\right|\right)^{1+\rho} \\
& \lesssim \varepsilon+|\Lambda|\left|\Lambda_{\ell}\right|^{\rho}|\Lambda|^{-(1+\rho) /(1+\tilde{\rho})} \varepsilon^{(1+\rho) /(1+\tilde{\rho})} \\
& \lesssim \varepsilon .
\end{aligned}
$$

To obtain the second inequality, we have used the upper bound provided by (2.1) of Lemma 2.1. The third inequality is a consequence of assumption (1.42).

On the other hand, if $k_{1} \neq 0$, clearly, one has $e^{-\left|I_{1}\right|} \frac{\left|I_{1}\right|^{k_{1}}}{k_{1} !} \lesssim \varepsilon$; moreover, one always has $e^{-|I|} \frac{|I|^{k}}{k !} \leq$ 1. Thus, $e^{-\left|I_{1}\right|} \frac{\left|I_{1}\right|^{k_{1}}}{k_{1} !} \cdots e^{-\left|I_{p}\right|} \frac{\left|I_{p}\right|^{k_{p}}}{k_{p} !} \lesssim \varepsilon$.

If $k_{1}=0$, then, by the computation made above, we have that

$$
0 \leq \mathbb{P}\left(\Omega_{I_{2}, k_{2} ; \cdots ; I_{p}, k_{p}}^{\Lambda} \cap \mathcal{Z}_{\Lambda}\right)-\mathbb{P}\left(\Omega_{I_{1}, 0 ; I_{2}, k_{2} ; \cdots ; I_{p}, k_{p}}^{\Lambda} \cap \mathcal{Z}_{\Lambda}\right) \lesssim \varepsilon
$$

and $0 \leq e^{-\left|I_{2}\right|} \frac{\left|I_{1}\right|^{k_{2}}}{k_{2} !} \cdots e^{-\left|I_{p}\right|} \frac{\left|I_{p}\right|^{k_{p}}}{k_{p} !}-e^{-\left|I_{1}\right|} e^{-\left|I_{2}\right|} \frac{\left|I_{1}\right|^{k_{2}}}{k_{2} !} \cdots e^{-\left|I_{p}\right|} \frac{\left|I_{p}\right|^{k_{p}}}{k_{p} !} \lesssim \varepsilon$. Thus we are back to estimating $\mathbb{P}\left(\Omega_{I_{2}, k_{2} ; \cdots ; I_{p}, k_{p}}^{\Lambda}\right)-e^{-\left|I_{2}\right|} \frac{\left|I_{2}\right|^{k_{2}}}{k_{2} !} \cdots e^{-\left|I_{p}\right|} \frac{\left|I_{p}\right|^{k_{p}}}{k_{p} !}$. 
So, from now on, we assume that all the intervals $\left(I_{j}\right)_{j}$ have length large than $\varepsilon$. Define $I_{j}^{+}=$ $I_{j} \cup\left[-e^{-L^{\eta}}, e^{-L^{\eta}}\right]$ and $I_{j}^{-}=I_{j} \cap\left({ }^{c} I_{j}+\left[-e^{-L^{\eta}}, e^{-L^{\eta}}\right]\right)$. Clearly, $I_{j}^{-} \subset I_{j} \subset I_{j}^{+}$. Moreover, by (1.46), for $L$ sufficiently large, we have that $I_{j}^{+} \cap I_{k}^{+}=I_{j}^{-} \cap I_{k}^{-}=\emptyset$ for $j<k$ and $\left|I_{j}^{+}\right|=\left|I_{j}\right|\left(1+O\left(e^{-L^{\eta}}\right)\right)$ and $\left|I_{j}^{-}\right|=\left|I_{j}\right|\left(1+O\left(e^{-L^{\eta}}\right)\right)$. For $L$ sufficiently large, one has $\left|I_{j}^{+}\right| \geq\left|I_{j}^{-}\right| \geq \varepsilon / 2$ for $j \in\{1, \ldots, p\}$. By Theorem 1.1, in particular (1.12), and the Wegner estimate (W), we know that

$$
\begin{aligned}
& \bigcap_{l=1}^{p}\left\{\omega ; \#\left\{j ; X_{\Lambda_{\ell}\left(\gamma_{j}\right), I_{l}^{-}}=1\right\}=k_{l}\right\} \bigcap \mathcal{Z}_{\Lambda} \subset \Omega_{I_{1}, k_{1} ; I_{2}, k_{2} ; \cdots ; I_{p}, k_{p}}^{\Lambda} \cap \mathcal{Z}_{\Lambda}, \\
& \Omega_{I_{1}, k_{1} ; I_{2}, k_{2} ; \cdots ; I_{p}, k_{p}}^{\Lambda} \cap \mathcal{Z}_{\Lambda} \subset \bigcap_{l=1}^{p}\left\{\omega ; \#\left\{j ; X_{\Lambda_{\ell}\left(\gamma_{j}\right), I_{l}^{+}}=1\right\}=k_{l}\right\} \bigcap \mathcal{Z}_{\Lambda} .
\end{aligned}
$$

Let us first use this to upper bound $\mathbb{P}\left(\Omega_{I_{1}, k_{1} ; I_{2}, k_{2} ; \cdots ; I_{p}, k_{p}}^{\Lambda} \cap \mathcal{Z}_{\Lambda}\right)$ and to show that we may assume $p$ to be finite (depending on $\varepsilon$ ). Therefore, we compute

$$
\begin{aligned}
\mathbb{P}\left(\Omega_{I_{1}, k_{1} ; I_{2}, k_{2} ; \cdots ; I_{p}, k_{p}}^{\Lambda} \cap \mathcal{Z}_{\Lambda}\right) \leq \mathbb{P}\left(\bigcap_{l=1}^{p}\left\{\omega ; \#\left\{j ; X_{\Lambda_{\ell}\left(\gamma_{j}\right), I_{l}^{+}}=1\right\}=k_{l}\right\} \cap \mathcal{Z}_{\Lambda}\right) \\
\leq \sum_{\substack{K_{l} \in\{1, \cdots, \tilde{N}\} \\
\text { \#K }=k_{l}, i \leq l \leq p \\
K_{l} \cap K_{l^{\prime}}=\emptyset \text { if } l \neq l^{\prime}}} \mathbb{P}\left(\bigcap_{l=1}^{p}\left\{\omega ; \begin{array}{c}
\forall j \in K_{l}, X_{\Lambda_{\ell}\left(\gamma_{j}\right), I_{l}^{+}}=1, \\
\forall j \notin K_{l}, X_{\Lambda_{\ell}\left(\gamma_{j}\right), I_{l}^{+}}=0
\end{array}\right\} \cap \mathcal{Z}_{\Lambda}\right)
\end{aligned}
$$

as, by the definition of $\mathcal{Z}_{\Lambda}$ (see Theorem 1.1), one has $\mathbb{P}\left(\left\{X_{\Lambda_{\ell}\left(\gamma_{j}\right), I_{l}^{+}}=1, X_{\Lambda_{\ell}\left(\gamma_{j}\right), I_{l^{\prime}}^{+}}=1\right\} \cap \mathcal{Z}_{\Lambda}\right)=0$ if $l \neq l^{\prime}$. As $K_{l} \cap K_{l^{\prime}}=\emptyset$ if $l \neq l^{\prime}$ the random vectors $\left(\left(X_{\Lambda_{\ell}\left(\gamma_{j}\right), I_{l}^{+}}\right)_{j \in K_{l}}\right)_{1 \leq l \leq p}$ are independent. Thus, one estimates

$$
\begin{aligned}
& \mathbb{P}\left(\Omega_{I_{1}, k_{1} ; I_{2}, k_{2} ; \cdots ; I_{p}, k_{p}}^{\Lambda} \cap \mathcal{Z}_{\Lambda}\right) \leq \sum_{\substack{K_{l} \in\{1, \cdots, \tilde{N}\} \\
\# K_{l}=k_{l}, 1 \leq l \leq p \\
K_{l} \cap K_{l^{\prime}}=\emptyset \text { if } l \neq l^{\prime}}} \prod_{l=1}^{p} \mathbb{P}\left(\left\{\omega ; \forall j \in K_{l}, X_{\Lambda_{\ell}\left(\gamma_{j}\right), I_{l}^{+}}=1\right\}\right) \\
& \times \mathbb{P}\left(\left\{\omega ; \forall j \notin \bigcup_{l=1}^{p} K_{l}, \sum_{l=1}^{p} X_{\Lambda_{\ell}\left(\gamma_{j}\right), I_{l}^{+}}=0\right\}\right) \\
& \leq \sum_{\substack{K_{l} \in\{1, \cdots, \tilde{N}\} \\
\# K_{l}=k_{l}, 1 \leq l \leq p}} \prod_{l=1}^{p} \mathbb{P}\left(\left\{\omega ; \forall j \in K_{l}, X_{\Lambda_{\ell}\left(\gamma_{j}\right), I_{l}^{+}}=1\right\}\right) \\
& \times \mathbb{P}\left(\left\{\omega ; \forall j \notin \bigcup_{l=1}^{p} K_{l}, \sum_{l=1}^{p} X_{\Lambda_{\ell}\left(\gamma_{j}\right), I_{l}^{+}}=0\right\}\right) \\
& \leq\left(\begin{array}{c}
\tilde{N} \\
k_{1}+\cdots+k_{p}
\end{array}\right) \prod_{l=1}^{p}\left(p_{l}^{+}\right)^{k_{l}} \\
& \times \mathbb{P}\left(\left\{\omega ; \sum_{l=1}^{p} X_{\Lambda_{\ell}\left(\gamma_{1}\right), I_{l}^{+}}=0\right\}\right)^{\tilde{N}-\left(k_{1}+\cdots+k_{p}\right)}
\end{aligned}
$$

where $p_{l}^{ \pm}=\mathbb{P}\left(X_{\Lambda_{\ell}\left(\gamma_{1}\right), I_{l}^{ \pm}}=1\right)$ for $1 \lambda \leq p$. Here, we have used the fact that, as the cubes $\left(\Lambda_{\ell}\left(\gamma_{j}\right)\right)_{j}$ are at distance at least $R$ from each other (see (IAD)), the events $\left(\left\{\omega ; \#\left\{j ; X_{\Lambda_{\ell}\left(\gamma_{j}\right), I_{l}^{-}}=1\right\}=k_{l}\right\}\right)_{j}$ 
are two by two independent for $1 \leq l \leq p$.

To estimate the last term in (4.4), we will use

Lemma 4.1. Set

$$
\delta:=\alpha \frac{1+\rho}{1+\tilde{\rho}}-1-\beta \rho .
$$

With the choice of $\left(I_{l}^{+}\right)_{1 \leq l \leq p}$ made above, under the assumptions of Theorem 1.10, with our choice of $\ell$ and $\ell^{\prime}$, possibly reducing $\beta$ somewhat, for $L$ sufficiently large, one has

$$
\mathbb{P}\left(\sum_{l=1}^{p} X_{\Lambda_{\ell}\left(\gamma_{1}\right), I_{l}^{+}}=0\right)=1-\frac{1-O\left(|\Lambda|^{-\delta}\right)}{\tilde{N}} \sum_{l=1}^{p}\left|I_{l}\right|
$$

Proof. The proof is analogous to that of Lemma 2.1; it also relies on our choice for the intervals $\left(I_{l}\right)_{1 \leq l \leq p}$. The derivation of (2.8) yields

$$
0 \leq \mathbb{E}\left[N\left(\bigcup_{j=1}^{p} N^{-1}\left(N\left(E_{0}\right)+|\Lambda|^{-1} I_{l}^{+}\right), \ell, \ell^{\prime}\right)\right]-\mathbb{P}\left(\sum_{l=1}^{p} X_{\Lambda_{\ell}\left(\gamma_{j}\right), I_{l}^{+}} \geq 1\right) \leq C\left|\Lambda_{\ell}\right|^{1+\rho}\left|I_{\Lambda}\right|^{1+\rho} .
$$

Thus using Lemma 2.2 and assumption (1.42), we obtain

$$
\begin{aligned}
\mathbb{P}\left(\sum_{l=1}^{p} X_{\Lambda_{\ell}\left(\gamma_{j}\right), I_{l}^{+}} \geq 1\right) & =N\left(\bigcup_{j=1}^{p} N^{-1}\left(N\left(E_{0}\right)+|\Lambda|^{-1} I_{l}^{+}\right)\right)+\left|\Lambda_{\ell}\right|^{1+\rho}\left|N\left(I_{\Lambda}\right)\right|^{(1+\rho) /(1+\tilde{\rho})} \\
& =|\Lambda|^{\beta-1} \bigcup_{j=1}^{p}\left|I_{l}^{+}\right|+O\left(|\Lambda|^{\beta(1+\rho)-\alpha(1+\rho) /(1+\tilde{\rho})}\right) .
\end{aligned}
$$

To conclude the statement of Lemma 4.1, it now suffices to recall that $\left|I_{l}^{+}\right| \geq \varepsilon / 2$ and that $1+\beta \rho<\alpha \frac{1+\rho}{1+\tilde{\rho}}$ i.e. $\beta-1>\beta(1+\rho)-\alpha \frac{1+\rho}{1+\tilde{\rho}}$. Moreover, by the definition of the intervals $\left(I_{l}^{+}\right)_{l}$, the main term in (4.6) is $|\Lambda|^{\beta-1} \bigcup_{j=1}^{p}\left|I_{l}\right|$.

By (2.1) of Lemma 2.1, the assumption (1.45) and the definition of $\left(I_{l}^{ \pm}\right)_{l}$, we have that

$$
p_{l}^{ \pm}=\left|I_{l}\right||\Lambda|^{\beta-1}\left(1+O\left(|\Lambda|^{-\delta}\right)\right)=\left|I_{l}\right| \tilde{N}^{-1}\left(1+O\left(|\Lambda|^{-\delta}\right)\right) .
$$

Setting $k=k_{1}+\cdots+k_{p}$ and $I^{+}=\left|I_{1}^{+}\right|+\cdots+\left|I_{1}^{+}\right|$, we note that by Theorem 1.1, for $\omega \in \mathcal{Z}_{\Lambda}$, we have $k \lesssim N\left(I_{\Lambda}\right)|\Lambda| \lesssim|\Lambda|^{1-\alpha}$ and, by the assumptions of Theorem 1.10, we have $I^{+} \leq 2|\Lambda|^{1-\alpha}$. Thus, for $L$ large, we have

$$
k+I^{+}=o\left(|\Lambda|^{1-\beta}\right)=o(\tilde{N}) .
$$

From (4.4), (4.7) and Lemma 4.1, at the possible expense of reducing $\beta$ somewhat, we, thus, obtain the following upper bound

$$
\begin{aligned}
\mathbb{P}\left(\Omega_{I_{1}, k_{1} ; I_{2}, k_{2} ; \cdots ; I_{p}, k_{p}}^{\Lambda} \cap \mathcal{Z}_{\Lambda}\right) & \leq\left(\begin{array}{c}
\tilde{N} \\
k
\end{array}\right)\left(\frac{I^{+}}{\tilde{N}}\right)^{k}\left(1-\frac{1-O\left(|\Lambda|^{-\delta}\right)}{\tilde{N}} I^{+}\right)^{\tilde{N}-k} \\
& \leq \frac{\left(I^{+}\right)^{k}}{k !} e^{-I^{+}} e^{O\left(|\Lambda|^{-\delta} I^{+}\right)} \lesssim \frac{\left(I^{+}\right)^{k}}{k !} e^{-I^{+}}
\end{aligned}
$$

where we have used (4.8) as well as the assumption (1.44) to obtain

$$
|\Lambda|^{-\delta} I^{+} \leq|\Lambda|^{-\delta+1-\alpha}=o(1)
$$


Indeed, by (4.5), the definition of $\delta$, and as $\alpha_{d, \rho, \tilde{\rho}}<\alpha$ (see (1.11)), one computes

$$
\begin{aligned}
-\delta+1-\alpha=-\alpha \frac{1+\rho}{1+\tilde{\rho}}+1+\beta \rho+1-\alpha & <-\frac{(2+\rho+\tilde{\rho})(1+d \rho)}{1+(d+1) \rho}+2+\beta \rho \\
& =-\frac{\tilde{\rho}(1+d \rho)-\rho(1-d \rho)}{1+(d+1) \rho}+\beta \rho
\end{aligned}
$$

So, under assumption (1.44), at the expense of possibly reducing $\beta$, one has $-\delta+1-\alpha<0$ which, taking into account (4.10), implies (4.9).

The bound (4.9) proves that if $k+I^{+} \rightarrow+\infty$ (as $\left.L \rightarrow+\infty\right)$ then $\mathbb{P}\left(\Omega_{I_{1}, k_{1} ; I_{2}, k_{2} ; \cdots ; I_{p}, k_{p}}^{\Lambda} \cap \mathcal{Z}_{\Lambda}\right) \rightarrow 0$. Note that, for $1 \leq l \leq p$, one has $\left|I_{l}^{+}\right| \geq \varepsilon / 2$, one has $\mathbb{P}\left(\Omega_{I_{1}, k_{1} ; I_{2}, k_{2} ; \cdots ; I_{p}, k_{p}}^{\Lambda} \cap \mathcal{Z}_{\Lambda}\right) \rightarrow 0$ if $p \rightarrow+\infty$ as $L \rightarrow+\infty$. On the other hand, 0 is clearly also the limit of the product

$$
\frac{\left|I_{1}\right|^{k_{1}}}{k_{1} !} \mathrm{e}^{-\left|I_{1}\right|} \ldots \frac{\left|I_{p}\right|^{k_{p}}}{k_{p} !} \mathrm{e}^{-\left|I_{p}\right|}
$$

in any of these cases (as $\left|I_{l}^{+}\right| \asymp\left|I_{l}\right| \geq \varepsilon / 2$ ). So we have proved (1.47) if $k+I^{+}+p \rightarrow+\infty$ when $L \rightarrow+\infty$.

From now on, we assume that $k, I^{+}$and $p$ are bounded. Let us prove (1.47) in this case. By (4.3), using the same computation as above, we have

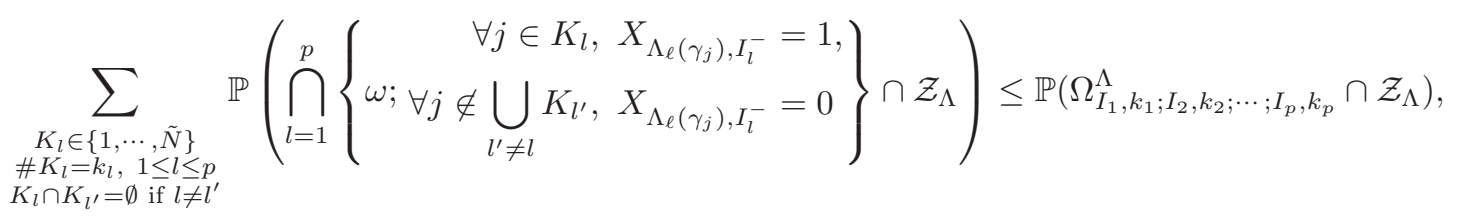

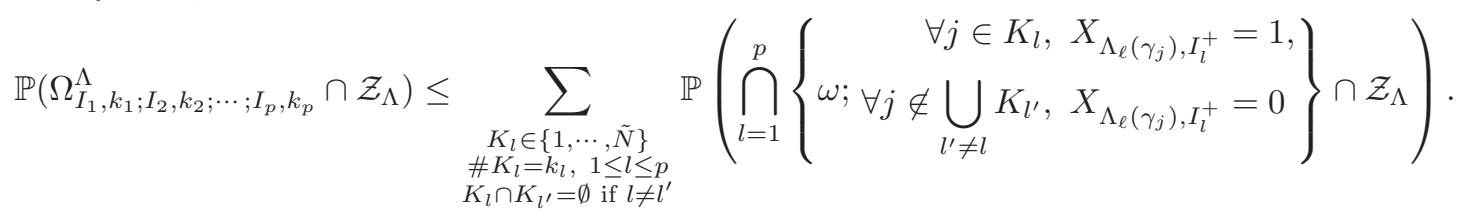

Hence, as $1-\mathbb{P}\left(\mathcal{Z}_{\Lambda}\right)=o(1)$ and $p$ is finite, one has

$$
\begin{aligned}
& \sum_{\substack{K_{l} \in\{1, \cdots, \tilde{N}\} \\
\forall K_{1}=k_{l}, 1 \leq l \leq p}} \mathbb{P}\left(\bigcap_{l=1}^{p}\left\{\omega ; \begin{array}{c}
\forall j \in K_{l}, X_{\Lambda_{\ell}\left(\gamma_{j}\right), I_{l}^{-}}=1, \\
\forall j \notin K_{l}, X_{\Lambda_{\ell}\left(\gamma_{j}\right), I_{l}^{-}}=0
\end{array}\right\}\right)+o(1) \leq \mathbb{P}\left(\Omega_{I_{1}, k_{1} ; I_{2}, k_{2} ; \cdots ; I_{p}, k_{p}}^{\Lambda} \cap \mathcal{Z}_{\Lambda}\right), \\
& \begin{array}{c}
\# K_{l}=k_{l}, 1 \leq l \leq p \\
K_{l} \cap K_{l^{\prime}}=\emptyset \text { if } l \neq l^{\prime}
\end{array} \\
& \mathbb{P}\left(\Omega_{I_{1}, k_{1} ; I_{2}, k_{2} ; \cdots ; I_{p}, k_{p}}^{\Lambda} \cap \mathcal{Z}_{\Lambda}\right) \leq \sum_{\substack{K_{l} \in\{1, \cdots, \tilde{N}\} \\
\# K_{l}=k_{l}, 1 \leq l \leq p \\
K_{l} \cap K_{l^{\prime}}=\emptyset \text { if } l \neq l^{\prime}}} \mathbb{P}\left(\bigcap_{l=1}^{p}\left\{\omega ; \begin{array}{c}
\forall j \in K_{l}, X_{\Lambda_{\ell}\left(\gamma_{j}\right), I_{l}^{+}}=1, \\
\forall j \notin K_{l}, X_{\Lambda_{\ell}\left(\gamma_{j}\right), I_{l}^{+}}=0
\end{array}\right\}\right)+o(1) .
\end{aligned}
$$


For $\left(K_{l}\right)_{1 \leq l \leq p} \in\{1, \cdots, \tilde{N}\}^{p}$ such that $\left(\# K_{l}\right)_{1 \leq l \leq p}=\left(k_{l}\right)_{1 \leq l \leq p}$ and $K_{l} \cap K_{l^{\prime}}=\emptyset$ if $l \neq l^{\prime}$, one computes

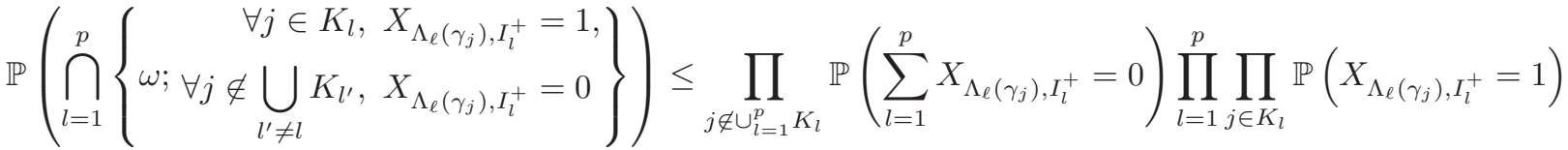

$$
\begin{aligned}
& \leq \mathbb{P}\left(\sum_{l=1}^{p} X_{\Lambda_{\ell}\left(\gamma_{1}\right), I_{l}^{+}}=0\right)^{\tilde{N}-\left(k_{1}+\cdots+k_{p}\right)} \prod_{l=1}^{p} \mathbb{P}\left(X_{\Lambda_{\ell}\left(\gamma_{1}\right), I_{l}^{+}}=1\right)^{k_{l}} \\
& \leq\left(1-\sum_{l=1}^{p} p_{l}^{+}\right)^{\tilde{N}-\left(k_{1}+\cdots+k_{p}\right)} \prod_{l=1}^{p}\left(\left(p_{l}^{+}\right)^{k_{l}}(1+o(1))\right. \\
& \leq\left(\prod_{l=1}^{p} e^{-\left|I_{l}^{+}\right|}\left|I_{l}^{+}\right|^{k_{l}}\right)(\tilde{N})^{k_{1}+\cdots+k_{p}}(1+o(1))
\end{aligned}
$$

and

$$
\begin{aligned}
\mathbb{P}\left(\bigcap_{l=1}^{p}\left\{\omega ; \quad \begin{array}{r}
\forall j \in K_{l}, X_{\Lambda_{\ell}\left(\gamma_{j}\right), I_{l}^{-}}=1, \\
\forall j \notin K_{l}, X_{\Lambda_{\ell}\left(\gamma_{j}\right), I_{l}^{-}}=0
\end{array}\right\}\right) \geq & \mathbb{P}\left(\sum_{l=1}^{p} X_{\Lambda_{\ell}\left(\gamma_{1}\right), I_{l}^{-}}=0\right)^{\tilde{N}-\left(k_{1}+\cdots+k_{p}\right)} \\
& \quad \times \prod_{j \in \bigcup_{l} K_{l}}\left(\sum_{l=1}^{p} \mathbf{1}_{j \in K_{l}} \mathbb{P}\left(\begin{array}{r}
X_{\Lambda_{\ell}\left(\gamma_{j}\right), I_{l}^{-}}=1, \\
\sum_{l^{\prime} \neq l} X_{\Lambda_{\ell}\left(\gamma_{j}\right), I_{l^{\prime}}^{-}}=0
\end{array}\right)\right) \\
\geq & \mathbb{P}\left(\sum_{l=1}^{p} X_{\Lambda_{\ell}\left(\gamma_{1}\right), I_{l}^{-}}=0\right)^{\tilde{N}-\left(k_{1}+\cdots+k_{p}\right)} \\
& \times \prod_{j \in \bigcup_{l} K_{l}}\left(\sum_{l=1}^{p} \mathbf{1}_{j \in K_{l}}\left[\mathbb{P}\left(X_{\Lambda_{\ell}\left(\gamma_{j}\right), I_{l}^{-}}=1\right)-\mathbb{P}\left(\begin{array}{c}
X_{\Lambda_{\ell}\left(\gamma_{j}\right), I_{l}^{-}}=1, \\
X_{\Lambda_{\ell}\left(\gamma_{j}\right), I_{l^{\prime}}}=1
\end{array}\right)\right]\right) \\
\geq & \left(\prod_{l=1}^{p} e^{-\left|I_{l}^{+}\right|}\left|I_{l}^{-}\right|^{k_{l}}\right)(\tilde{N})^{k_{1}+\cdots+k_{p}}(1+o(1))
\end{aligned}
$$

as $k_{1}+\cdot+k_{p}$ is bounded and

$$
\mathbb{P}\left(X_{\Lambda_{\ell}\left(\gamma_{j}\right), I_{l}^{-}}=1\right)-\mathbb{P}\left(\begin{array}{c}
X_{\Lambda_{\ell}\left(\gamma_{j}\right), I_{l}^{-}}=1, \\
X_{\Lambda_{\ell}\left(\gamma_{j}\right), I_{l^{\prime}}^{-}}=1
\end{array}\right)=p_{l}^{-}\left(1+O\left(|\Lambda|^{-\delta}\right)\right) .
$$

On the other hand, as $k_{1}+\cdots+k_{p}$ is bounded, when $N \rightarrow+\infty$, one has

$$
\sum_{\substack{K_{l} \in\{1, \ldots, \tilde{N}\} \\
\# K_{l}=k_{l}, \leq \leq l \leq p \\
K_{l} \cap K_{l^{\prime}}=\emptyset \text { if } l \neq l^{\prime}}} 1=\prod_{l=1}^{p}\left(\begin{array}{l}
\tilde{N} \\
k_{l}
\end{array}\right)(1+o(1))
$$

Thus, for $L$ sufficiently large, we obtain

$$
\begin{aligned}
e^{-\left|I_{1}^{-}\right|} \frac{\left|I_{1}^{-}\right|^{k_{1}}}{k_{1} !} \cdots e^{-\left|I_{p}^{-}\right|} \frac{\left|I_{p}^{-}\right|^{k_{p}}}{k_{p} !}(1+o(1)) & \leq \mathbb{P}\left(\Omega_{I_{1}, k_{1} ; I_{2}, k_{2} ; \cdots ; I_{p}, k_{p}}^{\Lambda} \cap \mathcal{Z}_{\Lambda}\right) \\
& \leq e^{-\left|I_{1}^{+}\right|} \frac{\left.I_{1}^{+}\right|^{k_{1}}}{k_{1} !} \cdots e^{-\left|I_{p}^{+}\right|} \frac{\left|I_{p}^{+}\right|^{k_{p}}}{k_{p} !}(1+o(1))
\end{aligned}
$$


Now, recalling that $\left|I_{l}^{ \pm}\right|=\left|I_{l}\right|+O\left(e^{-L^{\eta}}\right)$, we get

$$
\lim _{|\Lambda| \rightarrow+\infty}\left|\mathbb{P}\left(\Omega_{I_{1}, k_{1} ; I_{2}, k_{2} ; \cdots ; I_{p}, k_{p}}^{\Lambda}\right)-e^{-\left|I_{1}\right|} \frac{\left|I_{1}\right|^{k_{1}}}{k_{1} !} \cdots e^{-\left|I_{p}\right|} \frac{\left|I_{p}\right|^{k_{p}}}{k_{p} !}\right|=0
$$

and the proof of Theorem 1.10 is complete.

Remark 4.1. In the present proof, assumption (1.42) does not suffice to guarantee (4.7): indeed, as we did not fix the intervals $\left(I_{j}\right)_{j}$, we want a result uniform over all the intervals of not too small length in some neighborhood of $E_{0}$; thus, we use assumption (1.45), a uniform version of assumption (1.42).

To prove Theorem 1.9 however it suffices to consider fixed intervals $\left(I_{j}\right)_{1 \leq j \leq p}$; thus, assumption (1.42) suffices to guarantee that (4.7) holds.

4.1.2. The asymptotic independence. We now turn to the proof of Theorem 1.11. Decompose $\Lambda$ into the boxes constructed in Theorem 1.1 i.e. $\Lambda=\cup_{\gamma \in \Gamma_{\Lambda}} \Lambda_{\ell}(\gamma)$; the scale $\ell$ is determined by Theorem 1.1 and $N:=\# \Gamma_{\Lambda} \sim|\Lambda| \ell^{-d}$. The set of boxes thus obtained are the same for both energies $E$ and $E^{\prime}$. For $I \subset \mathbb{R}$ a compact set, define the random variables

$$
X_{\gamma}(E, I)=\left\{\begin{array}{l}
1 \text { if } H_{\omega}\left(\Lambda_{\ell}(\gamma)\right) \text { has an e.v. in } N^{-1}\left[N\left(E_{0}\right)+|\Lambda|^{-1} I\right] \\
0 \text { if not. }
\end{array}\right.
$$

Then, to prove Theorem 1.11, it suffices to prove that, for $\left(k, k^{\prime}\right) \in \mathbb{N}^{2}$ and $I \subset \mathbb{R}$ and $I^{\prime} \subset \mathbb{R}$ two compact sets, one has

$$
\begin{aligned}
\lim _{|\Lambda| \rightarrow+\infty} \mathbb{E}\left[\left(\sum_{\gamma \in \Gamma_{\Lambda}} X_{\gamma}(E, I)\right)^{k}\left(\sum_{\gamma \in \Gamma_{\Lambda}} X_{\gamma}\left(E^{\prime}, I^{\prime}\right)\right)^{k^{\prime}}\right] \\
=\lim _{|\Lambda| \rightarrow+\infty} \mathbb{E}\left[\left(\sum_{\gamma \in \Gamma_{\Lambda}} X_{\gamma}(E, I)\right)^{k}\right] \mathbb{E}\left[\left(\sum_{\gamma \in \Gamma_{\Lambda}} X_{\gamma}\left(E^{\prime}, I^{\prime}\right)\right)^{k^{\prime}}\right] .
\end{aligned}
$$

Therefore, using the independence of the cubes, we expand the sums

$$
\begin{aligned}
S_{N}\left(k, k^{\prime}\right)= & \mathbb{E}\left[\left(\sum_{\gamma \in \Gamma_{\Lambda}} X_{\gamma}(E, I)\right)^{k}\left(\sum_{\gamma \in \Gamma_{\Lambda}} X_{\gamma}\left(E^{\prime}, I^{\prime}\right)\right)^{k^{\prime}}\right] \\
& =\sum_{\gamma_{1}, \cdots, \gamma_{k}} \sum_{\gamma_{1}^{\prime}, \cdots, \gamma_{k^{\prime}}^{\prime}} \mathbb{E}\left[X_{\gamma_{1}}(E, I) \cdots X_{\gamma_{k}}(E, I) \cdot X_{\gamma_{1}^{\prime}}\left(E^{\prime}, I^{\prime}\right) \cdots X_{\gamma_{k^{\prime}}}\left(E^{\prime}, I^{\prime}\right)\right] \\
& =G_{N}\left(k, k^{\prime}\right)+R_{N}\left(k, k^{\prime}\right)
\end{aligned}
$$

where

$$
G_{N}\left(k, k^{\prime}\right)=\sum_{\left\{\gamma_{1}, \cdots, \gamma_{k}\right\} \cap\left\{\gamma_{1}^{\prime}, \cdots, \gamma_{k^{\prime}}^{\prime}\right\}=\emptyset} \mathbb{E}\left[X_{\gamma_{1}}(E, I) \cdots X_{\gamma_{k}}(E, I) \cdot X_{\gamma_{1}^{\prime}}\left(E^{\prime}, I^{\prime}\right) \cdots X_{\gamma_{k^{\prime}}^{\prime}}\left(E^{\prime}, I^{\prime}\right)\right]
$$


and

$$
\begin{aligned}
& R_{N}\left(k, k^{\prime}\right)=\sum_{\gamma} \sum_{\substack{\gamma_{1}, \cdots, \gamma_{l} \\
\gamma_{1}^{\prime}, \cdots, \gamma_{l^{\prime}}}} \mathbb{E}\left[X_{\gamma}(E, I) X_{\gamma}\left(E^{\prime}, I^{\prime}\right) \cdot \prod_{j=1}^{k-1} X_{\gamma_{j}}(E, I) \cdot \prod_{j^{\prime}=1}^{k^{\prime}-1} X_{\gamma_{j^{\prime}}}\left(E^{\prime}, I^{\prime}\right)\right] \\
&=\sum_{\gamma} \sum_{l=1}^{k-1} \sum_{k^{\prime}=1}^{k^{\prime}-1}\left(\begin{array}{c}
l \\
k-1
\end{array}\right)\left(\begin{array}{c}
l^{\prime} \\
k^{\prime}-1
\end{array}\right) \\
& \sum_{\substack{\gamma \notin\left\{\gamma_{1}, \cdots, \gamma_{l}\right\} \\
\gamma \notin\left\{\gamma_{1}^{\prime}, \cdots, \gamma_{l^{\prime}}\right\}}} \mathbb{E}\left[X_{\gamma}(E, I) X_{\gamma}\left(E^{\prime}, I^{\prime}\right) \cdot \prod_{j=1}^{l} X_{\gamma_{j}}(E, I) \cdot \prod_{j^{\prime}=1}^{l^{\prime}} X_{\gamma_{j^{\prime}}}\left(E^{\prime}, I^{\prime}\right)\right] \\
&=\sum_{\gamma} \mathbb{E}\left[X_{\gamma}(E, I) X_{\gamma}\left(E^{\prime}, I^{\prime}\right)\right] \sum_{l=1}^{k-1} \sum_{l^{\prime}=1}^{k^{\prime}-1}\left(\begin{array}{c}
l \\
k-1
\end{array}\right)\left(\begin{array}{c}
l^{\prime} \\
k^{\prime}-1
\end{array}\right) \\
& \sum_{\substack{\gamma \notin\left\{\gamma_{1}, \cdots, \gamma_{l}\right\} \\
\gamma \notin\left\{\gamma_{1}^{\prime}, \cdots, \gamma_{l^{\prime}}\right\}}} \mathbb{E}\left[\prod_{j=1}^{l} X_{\gamma_{j}}(E, I) \cdot \prod_{j^{\prime}=1}^{l^{\prime}} X_{\gamma_{j^{\prime}}}\left(E^{\prime}, I^{\prime}\right)\right] .
\end{aligned}
$$

Hence,

$$
R_{N}\left(k, k^{\prime}\right)=\sum_{\gamma} \mathbb{E}\left[X_{\gamma}(E, I) X_{\gamma}\left(E^{\prime}, I^{\prime}\right)\right] \sum_{l=1}^{k-1} \sum_{l^{\prime}=1}^{k^{\prime}-1}\left(\begin{array}{c}
l \\
k-1
\end{array}\right)\left(\begin{array}{c}
l^{\prime} \\
k^{\prime}-1
\end{array}\right) S_{N-1}\left(l, l^{\prime}\right) .
$$

On the other hand, one computes

$$
\begin{aligned}
S_{N}(k) S_{N}\left(k^{\prime}\right) & =\mathbb{E}\left[\left(\sum_{\gamma \in \Gamma_{\Lambda}} X_{\gamma}(E, I)\right)^{k}\right] \mathbb{E}\left[\left(\sum_{\gamma \in \Gamma_{\Lambda}} X_{\gamma}\left(E^{\prime}, I^{\prime}\right)\right)^{k^{\prime}}\right] \\
& =\sum_{\gamma_{1}, \cdots, \gamma_{k}} \mathbb{E}\left[X_{\gamma_{1}}(E, I) \cdots X_{\gamma_{k}}(E, I)\right] \sum_{\gamma_{1}^{\prime}, \cdots, \gamma_{k^{\prime}}^{\prime}} \mathbb{E}\left[X_{\gamma_{1}^{\prime}}\left(E^{\prime}, I^{\prime}\right) \cdots X_{\gamma_{k^{\prime}}}\left(E^{\prime}, I^{\prime}\right)\right] \\
& =G_{N}\left(k, k^{\prime}\right)+Q_{N}\left(k, k^{\prime}\right)
\end{aligned}
$$

where

$$
\begin{aligned}
& Q_{N}\left(k, k^{\prime}\right)= \sum_{\gamma} \sum_{\gamma_{1}, \cdots, \gamma_{k-1}} \mathbb{E}\left[X_{\gamma}(E, I) X_{\gamma_{1}}(E, I) \cdots X_{\gamma_{k-1}}(E, I)\right] \\
& \cdot \sum_{\gamma_{1}^{\prime}, \cdots, \gamma_{k^{\prime}-1}^{\prime}} \mathbb{E}\left[X_{\gamma}\left(E^{\prime}, I^{\prime}\right) X_{\gamma_{1}^{\prime}}\left(E^{\prime}, I^{\prime}\right) \cdots X_{\gamma_{k^{\prime}-1}^{\prime}}\left(E^{\prime}, I^{\prime}\right)\right] \\
&=\sum_{\gamma} \mathbb{E}\left[X_{\gamma}(E, I)\right] \mathbb{E}\left[X_{\gamma}\left(E^{\prime}, I^{\prime}\right)\right]\left[\sum_{l=1}^{k-1}\left(\begin{array}{c}
l \\
k-1
\end{array}\right) S_{N-1}(l)\right]^{2} .
\end{aligned}
$$

Hence,

$$
S_{N}\left(k, k^{\prime}\right)-S_{N}(k) S_{N}\left(k^{\prime}\right)=R_{N}\left(k, k^{\prime}\right)-Q_{N}\left(k, k^{\prime}\right) .
$$

By Cauchy-Schwartz, one has

$$
S_{N}\left(k, k^{\prime}\right) \leq \sqrt{S_{N}(2 k) S_{N}\left(2 k^{\prime}\right)}
$$


On the other hand, as $\mathbb{P}\left(X_{\gamma}=1\right) \leq C / N$ by Lemma 2.1 , one computes

$$
S_{N}(k) \leq \sum_{j=0}^{N}\left(\begin{array}{c}
j \\
N
\end{array}\right)\left(\frac{C}{N}\right)^{j} j^{k} \leq C_{k} \sum_{j=0}^{N}\left(\begin{array}{c}
j \\
N
\end{array}\right)\left(\frac{C k}{N}\right)^{j} \leq C_{k} e^{N \log (1+C k / N)} \leq C_{k} e^{C k}<+\infty
$$

where $C_{k}=e^{k(\log k-1-\log \log k)}$ for $k \geq 2$.

So, for any $k$ and $k^{\prime}$, one has

$$
\sup _{N \geq 1}\left|S_{N}(k)\right|+\left|S_{N}\left(k, k^{\prime}\right)\right|<+\infty
$$

Thus, using (4.13), (4.14) and (4.15), one obtains

$$
\begin{aligned}
& \left|S_{N}\left(k, k^{\prime}\right)-S_{N}(k) S_{N}\left(k^{\prime}\right)\right| \\
& \leq C_{k, k^{\prime}} \max \left(\sum_{\gamma} \mathbb{E}\left[X_{\gamma}(E, I)\right] \mathbb{E}\left[X_{\gamma}\left(E^{\prime}, I^{\prime}\right)\right], \sum_{\gamma} \mathbb{E}\left[X_{\gamma}(E, I) X_{\gamma}\left(E^{\prime}, I^{\prime}\right)\right]\right)
\end{aligned}
$$

Hence, (4.12) and thus Theorem 1.11 and 1.12 follow from the following two properties

$$
\sum_{\gamma} \mathbb{E}\left[X_{\gamma}(E, I)\right] \mathbb{E}\left[X_{\gamma}\left(E^{\prime}, I^{\prime}\right)\right] \underset{|\Lambda| \rightarrow+\infty}{\rightarrow} 0 \text { and } \sum_{\gamma} \mathbb{E}\left[X_{\gamma}(E, I) X_{\gamma}\left(E^{\prime}, I^{\prime}\right)\right] \underset{|\Lambda| \rightarrow+\infty}{\rightarrow} 0 .
$$

As the operators $\left(H_{\omega}\left(\Lambda_{\ell}(\gamma)\right)\right)_{\gamma}$ are i.i.d, this will be proved if we prove that

$$
\left(\frac{L}{\ell}\right)^{d} \mathbb{E}\left[X_{0}(E, I)\right] \mathbb{E}\left[X_{0}\left(E^{\prime}, I^{\prime}\right)\right] \underset{|\Lambda| \rightarrow+\infty}{\rightarrow} 0 \text { and }\left(\frac{L}{\ell}\right)^{d} \mathbb{E}\left[X_{0}(E, I) X_{0}\left(E^{\prime}, I^{\prime}\right)\right] \underset{|\Lambda| \rightarrow+\infty}{\rightarrow} 0 .
$$

The first limit in (4.16) is an immediate consequence of the Wegner estimate as $\ell / L \rightarrow 0$. The second limit in (4.16) clearly is a consequence of (D) if $E \neq E^{\prime}$ are fixed energies, and of (GM) if $E=E_{\Lambda}$ and $E^{\prime}=E_{\Lambda}^{\prime}$ satisfy the assumptions of Theorem 1.12.

This completes the proof of Theorem 1.11 and 1.12.

4.2. Study of the (levels, centers) statistics. In this section, we will prove Theorems 1.13, 1.14 and 1.15. To control the eigenvalues, as in the previous section, we use Theorem 1.1. So we keep the same notations here.

4.2.1. The proof of Theorem 1.13. The proof of Theorem 1.13 is very similar to that of Theorem 1.10. One deals with the case when the length of some interval $\left(I_{k}\right)_{k}$ tend to 0 or $\infty$ as in the proof of Theorem 1.10; we will not repeat this here. We only indicate the differences and keep the same notations. We need to estimate the probability of the event

$$
\Omega_{\left(I_{l}, C_{l}, k_{l}\right)_{1 \leq l \leq p}}^{\Lambda}:=\bigcap_{l=1}^{p}\left\{\omega ; \#\left\{j ; \begin{array}{r}
\xi_{j}(\omega, \Lambda) \in I_{l} \\
x_{j}(\omega, \Lambda) / L \in C_{l}
\end{array}\right\}=k_{l}\right\} .
$$

Let us first deal with the case when the volume of one of the cubes $\left(C_{k}\right)_{k}$ tends to 0; assume now that $\left|C_{1}\right| \leq \varepsilon,\left|I_{1}\right| \geq \varepsilon$ and that $k_{1} \geq 1$. Then, we have that

$$
\begin{aligned}
\mathbb{P}\left(\Omega_{\left(I_{l}, C_{l}, k_{l}\right)_{1 \leq l \leq p}}^{\Lambda} \cap \mathcal{Z}_{\Lambda}\right) & \leq \mathbb{P}\left(\Omega_{I_{1}, C_{1}, k_{1}}^{\Lambda} \cap \mathcal{Z}_{\Lambda}\right) \\
& \lesssim \#\left\{\gamma \in L C_{1} ; \Lambda_{\ell}(\gamma) \text { in decomposition }\right\} \mathbb{P}\left(X_{\Lambda_{\ell}(\gamma), I_{1}+e^{-L^{\eta}}[-1,1]}=1\right) \\
& \lesssim L^{d}\left|C_{1}\right| \tilde{N} \cdot \ell^{d} \\
& \lesssim \varepsilon .
\end{aligned}
$$


The case when $k_{1}=0$ is then dealt with as in the proof of Theorem 1.10.

As in the proof of Theorem 1.10, one shows that if

$$
p+\left(k_{1}+\cdots+k_{p}\right)+\left(\left|I_{1}\right|+\cdots+\left|I_{p}\right|\right)+\left(\left|C_{1}\right|+\cdots+\left|C_{p}\right|\right) \rightarrow+\infty
$$

as $L \rightarrow+\infty$, then, both terms in (1.50) converge to 0 in the large $L$ limit.

The degenerate cases having been removed, the same reasoning as in the proof of Theorem 1.10 yields

$$
\begin{aligned}
\prod_{l=1}^{p} \mathbb{P}(\{\omega ; \#\{j ; & \left.\left.\left.\gamma_{j} / L \in C_{l}^{-} \text {and } X_{\Lambda_{\ell}\left(\gamma_{j}\right), I_{l}^{-}}=1\right\}=k_{l}\right\}\right)-\left(1-\mathbb{P}\left(\mathcal{Z}_{\Lambda}\right)\right) \\
& \leq \mathbb{P}\left(\Omega_{\left(I_{l}, C_{l}, k_{l}\right)_{1 \leq l \leq p}}^{\Lambda} \cap \mathcal{Z}_{\Lambda}\right) \\
& \leq \prod_{l=1}^{p} \mathbb{P}\left(\left\{\omega ; \#\left\{j ; \gamma_{j} / L \in C_{l}^{+} \text {and } X_{\Lambda_{\ell}\left(\gamma_{j}\right), I_{l}^{+}}=1\right\}=k_{l}\right\}\right)+\left(1-\mathbb{P}\left(\mathcal{Z}_{\Lambda}\right)\right)
\end{aligned}
$$

where $C_{l}^{+}=C_{l}+[-\ell / L, \ell / L]^{d}$ and $\mathbb{R}^{d} \backslash C_{l}^{-}=\mathbb{R}^{d} \backslash\left(C_{l}+(-\ell / L, \ell / L)^{d}\right)$.

Hence, the same computations as in the proof of Theorem 1.10 also yields

$$
\begin{aligned}
& \prod_{l=1}^{p}\left(\begin{array}{c}
\tilde{N}_{l}^{-} \\
k_{l}
\end{array}\right)\left(p_{l}^{-}\right)^{k_{l}}\left(1-p_{l}^{-}\right)^{\tilde{N}_{l}^{-}-k_{l}}+o(1) \leq \mathbb{P}\left(\Omega_{\left(I_{l}, C_{l}, k_{l}\right)_{1 \leq l \leq p}}^{\Lambda} \cap \mathcal{Z}_{\Lambda}\right) \\
& \leq \prod_{l=1}^{p}\left(\begin{array}{c}
\tilde{N}_{l}^{+} \\
k_{l}
\end{array}\right)\left(p_{l}^{+}\right)^{k_{l}}\left(1-p_{l}^{+}\right)^{\tilde{N}_{l}^{+}-k_{l}}+o(1)
\end{aligned}
$$

where

$$
N_{l}^{+}=\left|C_{l}\right| \tilde{N}\left(1+O\left(L^{-1+\beta}\right)\right), \quad N_{l}^{-}=\left|C_{l}\right| \tilde{N}\left(1+O\left(L^{-1+\beta}\right)\right) .
$$

One concludes in the same way as in the proof of Theorem 1.10. This completes the proof of Theorem 1.13.

4.2.2. The proof of Theorem 1.14. Let $\ell_{\Lambda}$ be the scale defined in section 1.8 satisfying (1.52). To prove Theorem 1.14, it is sufficient to prove that, for $\left(I_{j}\right)_{1 \leq j \leq l}$ disjoint segments of $\mathbb{R}$ and $\left(C_{j}\right)_{1 \leq j \leq l}$ disjoint compact cubes in $\left[-c_{\ell}, c_{\ell}\right]$ (see $\left.(1.54)\right)$, one has

$$
\begin{array}{r}
\mathbb{P}\left(\forall 1 \leq j \leq l, \#\left\{\begin{array}{r}
N\left(E_{n}(\omega, \Lambda)\right) \\
x_{n}(\omega) \in \ell_{\Lambda} C_{j}\left(E_{0}\right)+\ell_{\Lambda}^{-d} I_{j}
\end{array}\right\} \geq k_{j}\right) \\
\quad \underset{|\Lambda| \rightarrow+\infty}{\rightarrow} \prod_{j=1}^{l}\left(\sum_{k \geq k_{j}} e^{-\left|I_{j}\right|\left|C_{j}\right|} \frac{\left(\left|I_{j}\right|\left|C_{j}\right|\right)^{k}}{k !}\right) .
\end{array}
$$

This is a consequence of Proposition 1.1 and Theorem 1.13. Indeed, we first pick $\left(C_{j}^{ \pm}\right)$cubes s.t. $\overline{C_{j}^{-}} \subset \stackrel{\circ}{C_{j}} \subset \overline{C_{j}} \subset \stackrel{\circ}{C_{j}^{+}}$and $\overline{C_{j}^{+}} \cap \overline{C_{k}^{+}}=\emptyset$ for $j \neq k$. For $\ell_{\Lambda}$ large, the cubes $\left(\ell_{\Lambda} \overline{C_{j}^{+}}\right)_{1 \leq j \leq l}$ are at distance at least $R$ from one another (see (IAD)), thus, the $\left(H_{\omega}\left(\ell_{\Lambda} C_{j}\right)\right)_{1 \leq j \leq l}$ are two by two 
independent so as $\left(H_{\omega}\left(\ell_{\Lambda} C_{j}^{-}\right)\right)_{1 \leq j \leq l}$ and $\left(H_{\omega}\left(\ell_{\Lambda} C_{j}^{+}\right)\right)_{1 \leq j \leq l}$. Using Proposition 1.1, we have that

$$
\begin{aligned}
\prod_{j=1}^{l} \mathbb{P}(\#\{n ; & \left.\left.N\left(E_{n}\left(\omega, \ell_{\Lambda} C_{j}^{-}\right)\right) \in N\left(E_{0}\right)+\ell_{\Lambda}^{-d} I_{j}^{-}\right\} \geq k_{j}\right)-\left(1-\mathbb{P}\left(\mathcal{Z}_{\Lambda}\right)\right)-\mathbb{P}_{-} \\
& \leq \mathbb{P}\left(\forall 1 \leq j \leq l, \#\left\{\begin{array}{c}
N\left(E_{n}(\omega, \Lambda)\right) \in N\left(E_{0}\right)+\ell_{\Lambda}^{-d} I_{j} \\
x_{n}(\omega) \in \ell_{\Lambda} C_{j}
\end{array}\right\} \geq k_{j}\right) \\
& \leq \prod_{j=1}^{l} \mathbb{P}\left(\#\left\{n ; N\left(E_{n}\left(\omega, \ell_{\Lambda} C_{j}^{+}\right)\right) \in N\left(E_{0}\right)+\ell_{\Lambda}^{-d} I_{j}^{+}\right\} \geq k_{j}\right)+\left(1-\mathbb{P}\left(\mathcal{Z}_{\Lambda}\right)\right)+\mathbb{P}_{+}
\end{aligned}
$$

where

$$
\mathbb{P}_{ \pm}:=\mathbb{P}\left\{\exists j ; \begin{array}{l}
H_{\omega}\left(\ell_{\Lambda} C_{j}\right) \text { has an eigenvalue in } \ell_{\Lambda}^{-d} I_{j} \\
\text { with a localiz. center in } \ell_{\Lambda}\left(C_{j}^{ \pm} \Delta C_{j}\right)
\end{array}\right\} \lesssim \sum_{j=1}^{l}\left|C_{j}^{ \pm} \Delta C_{j}\right|,
$$

the last bound being a consequence of the Wegner estimate (W).

Now, using Theorem 1.13, we compute the asymptotics of $\mathbb{P}\left(\#\left\{n ; N\left(E_{n}\left(\omega, \ell_{\Lambda} C_{j}^{ \pm}\right)\right) \in N\left(E_{0}\right)+\right.\right.$ $\left.\left.\ell_{\Lambda}^{-d} I_{j}^{ \pm}\right\}\right)$; then, we let $C_{j}^{ \pm}$tend to $C_{j}$ and $I_{j}^{ \pm}$tend to $I_{j}$ to get the desired result. This completes the proof of Theorem 1.14.

4.2.3. The proof of Theorem 1.15. It is sufficient to consider the case of $J$ a (non empty) segment and $C$ a (non empty) cube. As the operators we considered are defined with periodic boundary conditions, we can restrict ourselves to cubes containing 0 . We can also assume $J$ is of the form $[0, a]$ or $[-a, 0]$ for some $a>0$. Hence, the sets $\tilde{\ell}_{\Lambda} C$ are increasing and the sets $E_{0}+\ell_{\Lambda} J$ are decreasing.

Pick $\ell^{ \pm}=\left(\ell_{\Lambda}^{ \pm}\right)_{\Lambda}$ and $\tilde{\ell}^{ \pm}=\left(\tilde{\ell}_{\Lambda}^{ \pm}\right)_{\Lambda}$ such that

$$
\pm \frac{\ell_{\Lambda}^{ \pm}-\ell_{\Lambda}}{\log ^{1 / \xi}|\Lambda|} \rightarrow+\infty, \quad \frac{\ell_{\Lambda}^{ \pm}}{\ell_{\Lambda}} \rightarrow 1, \quad \pm \frac{\tilde{\ell}_{\Lambda}^{ \pm}-\tilde{\ell}_{\Lambda}}{\log ^{1 / \xi}|\Lambda|} \rightarrow+\infty, \quad \frac{\tilde{\ell}_{\Lambda}^{ \pm}}{\tilde{\ell}_{\Lambda}} \rightarrow 1
$$

Let $\chi_{A}$ be the characteristic function of $A$. Compute

$$
\operatorname{tr}\left(\chi_{\tilde{\ell}_{\Lambda} C} \mathbf{1}_{N^{-1}\left[N\left(E_{0}\right)+\left(\ell_{\Lambda}\right)^{-d} J\right]}\left(H_{\omega}(\Lambda)\right)\right)=\sum_{\substack{E_{n}(\omega, \Lambda) \in \sigma\left(H_{\omega}(\Lambda)\right) \\ N\left(E_{n}(\omega, \Lambda)\right) \in N\left(E_{0}\right)+\ell_{\Lambda}^{-d} J}}\left\|\chi_{\tilde{\ell}_{\Lambda} C} \varphi_{n}(\omega, \Lambda)\right\|^{2} .
$$

If $\varphi_{n}(\omega, \Lambda)$ has its localization center in $\tilde{\ell}_{\Lambda} C$, by (4.18) and (1.7), one has

$$
\left\|\chi_{\tilde{\ell}_{\Lambda}^{+} C} \varphi_{n}(\omega, \Lambda)\right\|^{2}=1+O\left(|\Lambda|^{-\infty}\right),
$$

and if it has its localization center outside $\tilde{\ell}_{\Lambda} C$, then

$$
\left\|\chi_{\tilde{\ell}_{\Lambda}^{-} C} \varphi_{n}(\omega, \Lambda)\right\|^{2}=O\left(|\Lambda|^{-\infty}\right) .
$$

Hence, as the number of eigenvalues of $H_{\omega}(\Lambda)$ in $N^{-1}\left[N\left(E_{0}\right)+\left(\ell_{\Lambda}\right)^{-d} J\right]$ is bounded by $C|\Lambda|$ for some $C>0$, by Lemma 1.2, we get that, for any $p>0$, for $\Lambda$ sufficiently large, with a probability at least $1-|\Lambda|^{-p}$, one has

$$
\begin{aligned}
\int_{J \times C} \Xi_{\Lambda}^{2}\left(\xi, x ; E_{0}, \ell, \tilde{\ell}\right) d \xi d x-|\Lambda|^{-p} & \leq \operatorname{tr}\left(\chi_{\left.\tilde{\ell}_{\Lambda}^{+} C^{1} \mathbf{1}_{N^{-1}\left[N\left(E_{0}\right)+\left(\ell_{\Lambda}\right)^{-d} J\right]}\left(H_{\omega}(\Lambda)\right)\right)}\right. \\
& \leq \int_{J \times C} \Xi_{\Lambda}^{2}\left(\xi, x ; E_{0}, \ell, \tilde{\ell}\right) d \xi d x+|\Lambda|^{-p} .
\end{aligned}
$$


Partitioning $\tilde{\ell}_{\Lambda}^{ \pm} C$ into cubes of side length 1 and using the covariance and (4.18), for $\chi_{0}$ taken as in section 2 , we get that

$$
\begin{aligned}
\left(\tilde{\ell}_{\Lambda}\right)^{d}|C| \mathbb{E}_{\ell_{\Lambda}}(1+o(1)) \leq & \mathbb{E}\left(\operatorname{tr}\left(\chi_{\tilde{\ell}_{\Lambda}^{-} C^{1}} \mathbf{1}_{N^{-1}\left[N\left(E_{0}\right)+\left(\ell_{\Lambda}\right)^{-d} J\right]}\left(H_{\omega}(\Lambda)\right)\right)\right) \\
& \leq \mathbb{E}\left(\operatorname{tr}\left(\chi_{\tilde{\ell}_{\Lambda}^{+} C^{1}} \mathbf{1}_{N^{-1}\left[N\left(E_{0}\right)+\left(\ell_{\Lambda}\right)^{-d} J\right]}\left(H_{\omega}(\Lambda)\right)\right)\right) \leq\left(\tilde{\ell}_{\Lambda}\right)^{d}|C| \mathbb{E}_{\ell_{\Lambda}}(1+o(1)) .
\end{aligned}
$$

where $\mathbb{E}_{\ell_{\Lambda}}=\mathbb{E}\left(\operatorname{tr}\left(\chi_{0} \mathbf{1}_{N^{-1}\left[N\left(E_{0}\right)+\left(\ell_{\Lambda}\right)^{-d} J\right]}\left(H_{\omega}(\Lambda)\right)\right)\right)$. The computations done in section 2 show that

$$
\mathbb{E}_{\ell_{\Lambda}}=\left(\ell_{\Lambda}\right)^{-d}|J|(1+o(1)) .
$$

Taking the expectation in (4.19), we immediately obtain

$$
\begin{gathered}
\mathbb{E}\left(\int_{J \times C} \Xi_{\Lambda}^{2}\left(\xi, x ; E_{0}, \ell, \tilde{\ell}\right) d \xi\right) \leq C\left(\frac{\tilde{\ell}_{\Lambda}}{\ell_{\Lambda}}\right)^{d} \text { if } \frac{\tilde{\ell}_{\Lambda}}{\ell_{\Lambda} \underset{|\Lambda| \rightarrow+\infty}{\rightarrow} 0,} \\
\left(\frac{\ell_{\Lambda}}{\tilde{\ell}_{\Lambda}}\right)^{d} \mathbb{E}\left(\int_{J \times C} \Xi_{\Lambda}^{2}\left(\xi, x ; E_{0}, \ell, \tilde{\ell}\right) d \xi\right) \underset{|\Lambda| \rightarrow+\infty}{\rightarrow}|J| \cdot|C| \text { if } \frac{\tilde{\ell}_{\Lambda}}{\ell_{\Lambda}} \underset{|\Lambda| \rightarrow+\infty}{\rightarrow}+\infty .
\end{gathered}
$$

Assume now that $\frac{\tilde{\ell}_{\Lambda}}{\ell_{\Lambda}} \geq|\Lambda|^{\rho}$. Pick two scales $\left(\ell_{\Lambda}^{\prime}\right)_{\Lambda}$ and $\left(\ell_{\Lambda}^{\prime \prime}\right)_{\Lambda}$ such that, for some $\rho^{\prime}>0$

$$
\ell_{\Lambda}^{\prime} \geq|\Lambda|^{\rho^{\prime}}, \quad \frac{\tilde{\ell}_{\Lambda}}{\ell_{\Lambda}^{\prime}} \geq|\Lambda|^{\rho^{\prime}} \quad \text { and } \quad \frac{\ell_{\Lambda}^{\prime}}{\ell_{\Lambda}} \geq|\Lambda|^{\rho^{\prime}} .
$$

Partition the cubes $\tilde{\ell}_{\Lambda}^{ \pm} C$ into cubes of side length asymptotic to $\ell_{\Lambda}^{\prime}$ : let $\Gamma_{\Lambda}^{ \pm}=\left(\ell_{\Lambda}^{\prime} \mathbb{Z}^{d}\right) \cap\left(\tilde{\ell}_{\Lambda}^{ \pm} C\right)$ and

$$
\tilde{\ell}_{\Lambda}^{ \pm} C=\bigcup_{\gamma \in \Gamma_{\Lambda}} C_{\gamma, \ell_{\Lambda}^{\prime}} \quad \text { where } \quad C_{\gamma, \ell_{\Lambda}^{\prime}}^{ \pm}=\gamma+\ell_{\Lambda}^{\prime}[-1 / 2,1 / 2]^{d} .
$$

Then, we have

$$
\operatorname{tr}\left(\chi_{\tilde{\ell}_{\Lambda}^{ \pm} C} \mathbf{1}_{N^{-1}\left[N\left(E_{0}\right)+\left(\ell_{\Lambda}\right)^{-d} J\right]}\left(H_{\omega}(\Lambda)\right)\right)=\sum_{\gamma \in \Gamma_{\Lambda}^{ \pm}} \operatorname{tr}\left(\chi_{C_{\gamma, \ell_{\Lambda}^{\prime}}^{ \pm}} \mathbf{1}_{E_{0}+\left(\ell_{\Lambda}\right)^{-d} J}\left(H_{\omega}(\Lambda)\right)\right)
$$

Thus

$$
\operatorname{tr}^{2}\left(\chi_{\tilde{\ell}_{\Lambda}^{ \pm} C} \mathbf{1}_{N^{-1}\left[N\left(E_{0}\right)+\left(\ell_{\Lambda}\right)^{-d} J\right]}\left(H_{\omega}(\Lambda)\right)\right)=\sum_{\gamma \in \Gamma_{\Lambda}} \sum_{\gamma^{\prime} \in \Gamma_{\Lambda}} T\left(\gamma, J, \ell_{\Lambda}^{\prime}, \ell_{\Lambda}, \Lambda\right) T\left(\gamma^{\prime}, J, \ell_{\Lambda}^{\prime}, \ell_{\Lambda}, \Lambda\right)
$$

where

$$
T(\gamma)=T\left(\gamma, J, \ell_{\Lambda}^{\prime}, \ell_{\Lambda}, \Lambda\right)=\operatorname{tr}\left(\chi_{C_{\gamma, \ell_{\Lambda}^{\prime}}^{ \pm}} \mathbf{1}_{N^{-1}\left[N\left(E_{0}\right)+\left(\ell_{\Lambda}\right)^{-d} J\right]}\left(H_{\omega}(\Lambda)\right)\right)
$$

We prove

Lemma 4.2. If $\left|\gamma-\gamma^{\prime}\right| \geq 2 \ell_{\Lambda}^{\prime}$ then

$$
\left|\mathbb{E}\left(T(\gamma) \cdot T\left(\gamma^{\prime}\right)\right)-\mathbb{E}(T(\gamma)) \cdot \mathbb{E}\left(T\left(\gamma^{\prime}\right)\right)\right| \leq C e^{-\left(\ell_{\Lambda}^{\prime}\right)^{1 / \xi} / C} .
$$

Hence, we have

$$
\mathbb{E}\left|\operatorname{tr}\left(\chi_{\tilde{\ell}_{\Lambda}^{ \pm} C} \mathbf{1}_{N^{-1}\left[N\left(E_{0}\right)+\left(\ell_{\Lambda}\right)^{-d} J\right]}\left(H_{\omega}(\Lambda)\right)\right)-\mathbb{E}\left(\operatorname{tr}\left(\chi_{\tilde{\ell}_{\Lambda}^{ \pm} C} \mathbf{1}_{N^{-1}\left[N\left(E_{0}\right)+\left(\ell_{\Lambda}\right)^{-d} J\right]}\left(H_{\omega}(\Lambda)\right)\right)\right)\right|^{2} \leq C\left(\ell_{\Lambda}^{\prime}\right)^{d}
$$

By (4.22) and (4.21), we get, for some $\rho>0$,

$$
\mathbb{E}\left|\left(\frac{\ell_{\Lambda}}{\tilde{\ell}_{\Lambda}}\right)^{d} \operatorname{tr}\left(\chi_{\tilde{\ell}_{\Lambda}^{ \pm} C} \mathbf{1}_{N^{-1}\left[N\left(E_{0}\right)+\left(\ell_{\Lambda}\right)^{-d} J\right]}\left(H_{\omega}(\Lambda)\right)\right)-\right| J|\cdot| C||^{2} \leq C|\Lambda|^{-\rho}
$$


If $\tilde{\ell}_{\Lambda} / \ell_{\Lambda}^{\prime} \leq|\Lambda|^{-\rho},(4.20)$ becomes

$$
\mathbb{E}\left(\int_{J \times C} \Xi_{\Lambda}^{2}\left(\xi, x ; E_{0}, \ell, \tilde{\ell}\right) d \xi\right) \leq C|\Lambda|^{-d \rho} .
$$

Now choose the scales $\tilde{\ell}_{\Lambda_{L} p}^{+}=\tilde{\ell}_{\Lambda_{(L+1)} p}$ and $\ell_{\Lambda_{L^{p}}}^{+}=\ell_{\Lambda_{(L+1)}{ }^{p}}$. By (1.56), $\tilde{\ell}_{\Lambda_{L^{p}}}^{+} / \tilde{\ell}_{\Lambda_{L^{p}}} \rightarrow 1$ and $\ell_{\Lambda_{L^{p}}}^{+} / \ell_{\Lambda_{L^{p}}} \rightarrow 1$. Moreover, the estimates (4.24) and (4.23) hold for the pairs of scales $\left(\tilde{\ell}_{\Lambda_{L^{p}}}^{+}, \ell_{\Lambda_{L^{p}}}\right)$, and $\left(\tilde{\ell}_{\Lambda_{L} p}, \ell_{\Lambda_{L^{p}}}^{+}\right)$. Moreover, for $p$ large enough, they are summable. Thus, we have proved that

- in case (1) of Theorem 1.15, $\omega$ almost surely, for $L$ sufficiently large,

$$
\int_{J \times C} \Xi_{\Lambda_{L}^{p}}^{2}\left(\xi, x ; E_{0}, \ell, \tilde{\ell}^{+}\right) d \xi d x=0 .
$$

- in case (2) of Theorem 1.15, $\omega$ almost surely,

$$
\begin{aligned}
& \left(\frac{\ell_{\Lambda_{L^{p}}}^{+}}{\tilde{\ell}_{\Lambda_{L^{p}}}}\right)^{-d} \int_{J \times C} \Xi_{\Lambda_{L^{p}}}^{2}\left(\xi, x ; E_{0}, \ell, \tilde{\ell}^{+}\right) d \xi d x \underset{|\Lambda| \rightarrow+\infty}{\rightarrow}|J| \cdot|C|, \\
& \left(\frac{\ell_{\Lambda_{L^{p}}}}{\tilde{\ell}_{\Lambda_{L^{p}}}^{+}}\right)^{-d} \int_{J \times C} \Xi_{\Lambda_{L^{p}}}^{2}\left(\xi, x ; E_{0}, \ell^{+}, \tilde{\ell}\right) d \xi d x \underset{|\Lambda| \rightarrow+\infty}{\rightarrow}|J| \cdot|C|
\end{aligned}
$$

For $L^{p} \leq k \leq(L+1)^{p}$, as the sequences $\left(\ell_{\Lambda}\right)_{\Lambda}$ and $\left(\tilde{\ell}_{\Lambda}\right)_{\Lambda}$ are increasing, one has

$$
\begin{aligned}
\int_{J \times C} \Xi_{\Lambda_{L^{p}}}^{2}\left(\xi, x ; E_{0}, \ell, \tilde{\ell}^{+}\right) d \xi d x & \geq \int_{J \times C} \Xi_{\Lambda_{k}}^{2}\left(\xi, x ; E_{0}, \ell, \tilde{\ell}\right) d \xi d x \\
& \geq \int_{J \times C} \Xi_{\Lambda_{L^{p}}}^{2}\left(\xi, x ; E_{0}, \ell^{+}, \tilde{\ell}\right) d \xi d x .
\end{aligned}
$$

By (1.56), for $L^{p} \leq k \leq(L+1)^{p}, \tilde{\ell}_{\Lambda_{L^{p}}}^{+} \sim \tilde{\ell}_{\Lambda_{k}}^{+} \sim \tilde{\ell}_{\Lambda_{L^{p}}}$ and $\ell_{\Lambda_{L^{p}}}^{+} \sim \ell_{\Lambda_{k}}^{+} \sim \ell_{\Lambda_{L^{p}}}$. Hence, we get

- in case (1) of Theorem 1.15, $\omega$ almost surely, for $L$ sufficiently large,

$$
\int_{J \times C} \Xi_{\Lambda}^{2}\left(\xi, x ; E_{0}, \ell, \tilde{\ell}^{+}\right) d \xi d x=0 .
$$

As $\int_{J \times C} \Xi_{\Lambda}^{2}\left(\xi, x ; E_{0}, \ell, \tilde{\ell}^{+}\right) d \xi d x$ is an integer, this implies that this integer is 0 .

- in case (2) of Theorem 1.15, $\omega$ almost surely,

$$
\left(\frac{\ell_{\Lambda}}{\tilde{\ell}_{\Lambda}}\right)^{-d} \int_{J \times C} \Xi_{\Lambda}^{2}\left(\xi, x ; E_{0}, \ell, \tilde{\ell}\right) d \xi d x \underset{|\Lambda| \rightarrow+\infty}{\rightarrow}|J| \cdot|C| .
$$

This completes the proof of Theorem 1.15.

Remark 4.2. If we don't assume that either $\tilde{\ell}_{\Lambda} / \ell_{\Lambda}^{\prime} \leq|\Lambda|^{-\rho}$ or $\tilde{\ell}_{\Lambda} / \ell_{\Lambda}^{\prime} \leq|\Lambda|^{-\rho}$, but merely that either tends to 0, or we do not assume condition (1.56) then, (4.20), (4.19) and (4.23) show nevertheless that

$$
\begin{gathered}
\mathbb{E}\left(\int_{J \times C} \Xi_{\Lambda}^{2}\left(\xi, x ; E_{0}, \ell, \tilde{\ell}\right) d \xi\right) \rightarrow 0 \quad \text { if } \quad \frac{\tilde{\ell}_{\Lambda}}{\ell_{\Lambda}} \underset{|\Lambda| \rightarrow+\infty}{\rightarrow} 0, \\
\mathbb{E}\left|\left(\frac{\ell_{\Lambda}}{\tilde{\ell}_{\Lambda}}\right)^{d} \int_{J \times C} \Xi_{\Lambda}^{2}\left(\xi, x ; E_{0}, \ell, \tilde{\ell}\right) d \xi-\right| J|\cdot| C||^{2} \rightarrow 0 \quad \text { if } \quad \frac{\tilde{\ell}_{\Lambda}}{\ell_{\Lambda}|\Lambda| \rightarrow+\infty} \rightarrow \infty .
\end{gathered}
$$

This implies convergence in probability. 
Proof of Lemma 4.2. Define $\tilde{C}_{\gamma, \ell_{\Lambda}^{\prime}}^{ \pm}=C_{\gamma, \ell_{\Lambda}^{\prime}}^{ \pm}+\ell_{\Lambda}^{\prime}[-1 / 2,1 / 2]^{d}$; hence, in view of the computations done in the proof of Lemma 2.2 and in section 5.1, there exists $C>0$ such that, for $\delta>0$, we have

$$
\begin{aligned}
& \mathbb{E}\left|\operatorname{tr}\left(\chi_{C_{\gamma, \ell_{\Lambda}^{\prime}}} \mathbf{1}_{N^{-1}\left[N\left(E_{0}\right)+\left(\ell_{\Lambda}\right)^{-d} J\right]}\left(H_{\omega}(\Lambda)\right)\right)-\operatorname{tr}\left(\chi_{C_{\gamma, \ell_{\Lambda}^{\prime}}^{ \pm}} \mathbf{1}_{N^{-1}\left[N\left(E_{0}\right)+\left(\ell_{\Lambda}\right)^{-d} J\right]}\left(H_{\omega}\left(\tilde{C}_{\gamma, \ell_{\Lambda}^{\prime}}^{ \pm}\right)\right)\right)\right| \\
& \leq C\left(\delta\left(\ell_{\Lambda}\right)^{d}+\delta^{-C}\left(\ell_{\Lambda}\right)^{d C} e^{-\left(\ell_{\Lambda}^{\prime}\right)^{1 / \xi} / C}\right)
\end{aligned}
$$

Pick $\delta=e^{-\left(\ell_{\Lambda}^{\prime}\right)^{1 / \xi} / C^{\prime}}$ for some $C^{\prime}>C$. As $\left|\gamma-\gamma^{\prime}\right| \geq 2 \ell_{\Lambda}^{\prime}$, the operators $H_{\omega}\left(\tilde{C}_{\gamma, \ell_{\Lambda}^{\prime}}^{ \pm}\right)$and $H_{\omega}\left(\tilde{C}_{\gamma^{\prime}, \ell_{\Lambda}^{\prime}}^{ \pm}\right)$ are stochastically independent of each other. Now, as by standard arguments of Schrödinger operator theory (see e.g. [RS79]), $T(\gamma)$ and $T\left(\gamma^{\prime}\right)$ are bounded by $C\left(\ell_{\Lambda}^{\prime}\right)^{d},(4.25)$ yields the result of Lemma 4.2 and completes its proof.

4.3. Study of the level spacings statistics. We will first prove Theorem 1.4. To do so, we first use a the reduction constructed in Theorem 1.2 and study the spacings for the approximated eigenvalues given by Theorem 1.2. Then, we derive the statistics described in Theorem 1.4 from those computations. Using the estimates obtained in the proof of Theorem 1.4, we will prove Theorem 1.6.

4.3.1. Some preliminary considerations. We now use Theorem 1.2. The length scale $\ell_{\Lambda}^{\prime}$ (the localization radius) is determined by Theorem 1.2 i.e. $\ell^{\prime}=\ell_{\Lambda}^{\prime}=(R \log |\Lambda|)^{1 / \xi}$ where $\xi \in(0,1)$ can be chosen arbitrary by (Loc). Recall that $\rho^{\prime} \in[0, \rho /(1+d(\rho+1)))$ is fixed by assumption (1.25).

We first assume that the integrated density of states of the interval $E_{0}+I_{\Lambda}$, that is, $N\left(E_{0}+I_{\Lambda}\right)$ satisfies

$$
N\left(E_{0}+I_{\Lambda}\right) \asymp|\Lambda|^{-\alpha}
$$

for some $\alpha \in(0,1)$. When this is not the case, then by assumption (1.26), we know that $N\left(E_{0}+\right.$ $\left.I_{\Lambda}\right) \gg|\Lambda|^{-\alpha}$ for any $\alpha \in(0,1)$; thus, we can partition $E_{0}+I_{\Lambda}$ into intervals satisfying (4.26) for some $\alpha \in(0,1)$.

We pick the scale $\ell=\ell_{\Lambda}$ and so that,

$$
\ell_{\Lambda} \asymp N\left(E_{0}+I_{\Lambda}\right)^{-\nu} \text {. }
$$

We now show that $\nu$ can be chosen in $(0,1 / d)$ so that all the assumptions of Theorem 1.2 (in particular (1.13) and (1.17)) and Lemmas 2.1 and 2.3 (in particular (2.11)) are satisfied. Note that, when applying Lemmas 2.1 or 2.3 , in (2.11), the cube $\Lambda$ is the cube $\Lambda_{\ell}$.

These requirements yield the following conditions on the exponents

$$
\begin{aligned}
\frac{1}{1+\rho^{\prime}}>d \nu, & 0<1-\frac{1}{\alpha}+\frac{\rho-\rho^{\prime}}{1+\rho^{\prime}}-\nu d \rho, \\
\frac{\rho-\rho^{\prime}}{1+\rho^{\prime}}>\nu d(1+\rho), & 0<1-\frac{1}{\alpha}+\nu-\frac{\rho^{\prime}}{1+\rho^{\prime}} .
\end{aligned}
$$

As $\frac{1+\rho}{1+\rho^{\prime}}>1>\frac{\rho-\rho^{\prime}}{1+\rho^{\prime}}$, to obtain (4.28) for some $\nu \in(0,1 / d)$ and $\alpha \in(0,1)$, it suffices that

$$
\frac{\rho-\rho^{\prime}}{\left(1+\rho^{\prime}\right)(1+\rho)}>\frac{d \rho^{\prime}}{1+\rho^{\prime}}
$$

This is satisfied as $\rho^{\prime} \in[0, \rho /(1+d(\rho+1)))$.

Moreover, recalling the discussion following Lemma 2.1, we want $I_{\Lambda}$ and $\ell_{\Lambda}$ to be such that $\left(N\left(I_{\Lambda}\right)\left|\Lambda_{L}\right|\right)^{-1} \gg N\left(I_{\Lambda}\right)^{\left(\rho-\rho^{\prime}\right) /\left(1+\rho^{\prime}\right)}\left|\Lambda_{\ell_{\Lambda}}\right|^{\rho}$ that is, using (4.26) and (4.27), we need that

$$
\frac{1}{\alpha}<1+\frac{\rho-\rho^{\prime}}{1+\rho^{\prime}}-d \nu \rho .
$$


This condition is fulfilled by (4.28). From now on, we assume that $\ell=\ell_{\Lambda}$ and $N\left(E_{0}+I_{\Lambda}\right)$ satisfy (4.26) and (4.27) for such $\alpha$ and $\nu$. Pick $\beta \in(0,1 / 2)$ be such that

$$
\left(\frac{1}{\alpha}-1\right)(1+2 \beta)=\frac{\rho-\rho^{\prime}}{1+\rho^{\prime}}-d \nu \rho \text {. }
$$

Let $\mathcal{Z}_{\Lambda}$ be the set of realizations for which the conclusions of Theorem 1.2 hold. We know that, for any $p>0$, if $L$ is sufficiently large, one has $\mathbb{P}\left(\mathcal{Z}_{\Lambda}\right) \geq 1-O\left(|\Lambda|^{-p}\right)$ (see (1.15)).

Let $\tilde{N}$ be the number of the good cubes (i.e. cubes that determine eigenvalues of $H_{\omega}(\Lambda)$ ) constructed in Theorem 1.2 and $\left(\Lambda_{\ell}\left(\gamma_{j}\right)\right)_{1 \leq j \leq \tilde{N}}$ be those cubes. Then, $\tilde{N}=|\Lambda| \ell_{\Lambda}^{-d}(1+o(1))$. As before, define the following random variables:

- $X_{j}=X_{j}\left(\ell, E_{0}+I_{\Lambda}\right)$ is the Bernoulli random variable

$$
X_{j}=\mathbf{1}_{H_{\omega}\left(\Lambda_{\ell}\left(\gamma_{j}\right)\right) \text { has exactly one eigenvalue in } E_{0}+I_{\Lambda} \text { with localization center in } \Lambda_{\ell-\ell^{\prime}}} ;
$$

here, $\ell^{\prime} \asymp(\log |\Lambda|)^{1 / \xi} \ll \ell=\ell_{\Lambda}$ (see the discussion above);

- $\tilde{E}_{j}=\tilde{E}_{j}\left(\ell, E_{0}+I_{\Lambda}\right)$ is this eigenvalue conditioned on $X_{j}=1$.

Assume $I_{\Lambda}=\left[a_{\Lambda}, b_{\Lambda}\right]$ and define

$$
\xi_{j}=\frac{N\left(\tilde{E}_{j}\right)-N\left(E_{0}+a_{\Lambda}\right)}{N\left(E_{0}+b_{\Lambda}\right)-N\left(E_{0}+a_{\Lambda}\right)} .
$$

Note that $\xi_{j}$ is valued in $[0,1]$. Let $\Xi$ denote the common distribution function of the $\left(\xi_{j}\right)_{1 \leq j \leq k}$. It was studied in Lemma 2.3, the assumptions of which are satisfied (note that the set $\Lambda$ in (2.11) in Lemma 2.3 is the set $\left.\Lambda_{\ell}\left(\gamma_{j}\right)\right)$. The error term in $(2.12)$ is then of order $(\log |\Lambda|)^{-\beta}$ for some $\beta>0$. We first study the spacings for i.i.d. copies of the random variables $\left(\xi_{j}\right)_{1 \leq j \leq k}$. Let $\left(\bar{\xi}_{j}\right)_{1 \leq j \leq k}$ denote the $\left(\xi_{j}\right)_{1 \leq j \leq k}$ ordered increasingly and define

$$
D L S_{\xi}\left(x, k ; E_{0}+I_{\Lambda}, \omega, \Lambda\right)=\frac{1}{k-1} \#\left\{1 \leq j \leq k ; \bar{\xi}_{j+1}-\bar{\xi}_{j}>x / k\right\} .
$$

Our main technical result is

Lemma 4.3. Pick $E_{0} \in I$ such that (1.25) be satisfied. Pick $\left(I_{\Lambda}\right)_{\Lambda}$ be intervals and $\left(\ell_{\Lambda}\right)$ length scales such that (4.26) and (4.27) be satisfied for $(\nu, \alpha)$ satisfying (4.28). Define $\beta$ by (4.29) and let $N_{\Lambda}:=N\left(E_{0}+I_{\Lambda}\right)|\Lambda|$ and $K_{\Lambda}=N_{\Lambda}^{\beta}$.

Then, there exists $C>0$ such that, for $|\Lambda|$ sufficiently large, for $N_{\Lambda} K_{\Lambda}^{-1} \leq k \leq N_{\Lambda} K_{\Lambda}$, one

$$
\sup _{K_{\Lambda}^{-1} \leq x \leq K_{\Lambda}} \mathbb{E}\left(\left|D L S_{\xi}\left(x, k ; E_{0}+I_{\Lambda}, \omega, \Lambda\right)-D\left(k, E_{0}+I_{\Lambda}, \Lambda\right)\right|^{2}\right) \leq \frac{C}{k} .
$$

where we have defined

$$
D\left(k, E_{0}+I_{\Lambda}, \Lambda\right):=\int_{0}^{1}(1-\Xi(y+x / k)+\Xi(y))^{k-1} d \Xi(y) .
$$

Let $\left(J_{\Lambda}\right)_{\Lambda}$ be a sequence of intervals such that $\sup _{E \in J_{\Lambda}}|E| \rightarrow 0$ as $|\Lambda| \rightarrow+\infty$. Then

$$
\sup _{N_{\Lambda} K_{\Lambda}^{-1} \leq k \leq N_{\Lambda} K_{\Lambda}} \sup _{I_{\Lambda} \text { as in Lemma }} \operatorname{sum}_{4.3}\left|D\left(k, E_{0}+I_{\Lambda}, \Lambda\right)-e^{-x}\right| \underset{\mid \Lambda \| \rightarrow+\infty}{\rightarrow} 0 .
$$

Proof. To analyze the spacings of the $\left(\xi_{j}\right)_{1 \leq j \leq k}$, we use the computations of section 7 in [Pyk65] (see in particular (7.3)) that yield

$$
\int_{0}^{1}(1-\Xi(y+x / k)+\Xi(y))^{k-1} d \Xi(y)=\mathbb{E}\left(D L S_{\xi}\left(x, k ; E_{0}+I_{\Lambda}, \omega, \Lambda\right)\right)=D\left(k, E_{0}+I_{\Lambda}, \Lambda\right)
$$


and

$$
\begin{aligned}
& \mathbb{E}\left(\left[D L S_{\xi}\left(x, k ; E_{0}+I_{\Lambda}, \omega, \Lambda\right)\right]^{2}\right)=O\left(\frac{1}{k}\right)+ \\
& +2 \int_{\mathbb{R}} \int_{y+x / k}^{+\infty}(1-\Xi(y+x / k)+\Xi(y)-\Xi(z+x / k)+\Xi(z))^{k-2} d \Xi(z) d \Xi(y) .
\end{aligned}
$$

Fix $\nu \in(\xi, 1)$. By $(4.26)$ and $(4.27)$ as $\ell^{\prime}=\ell_{\Lambda}^{\prime} \asymp(\log |\Lambda|)^{1 / \xi}$, one has $K_{\Lambda}^{-1} \gg \ell_{\Lambda}^{d} e^{-\left(\ell_{\Lambda}^{\prime}\right)^{\nu}}$ for $|\Lambda|$ large. Thus, Lemma 2.3 and (4.29) yield that, for $y-x \geq\left(N_{\Lambda} K_{\Lambda}\right)^{-1}$, one has

$$
\Xi(y)-\Xi(x)=(y-x)\left(1+o\left(K_{\Lambda}^{-1}\right)\right)
$$

Hence, for $K_{\Lambda}^{-1} \leq x \leq K_{\Lambda}$ and $N_{\Lambda} K_{\Lambda}^{-1} \leq k \leq N_{\Lambda} K_{\Lambda}$, one has

$$
\begin{aligned}
& \mathbb{E}\left(D L S_{\xi}^{2}\left(x, k ; I_{\Lambda}, \omega, \Lambda\right)\right) \\
& =\int_{0}^{1} \int_{0}^{1}(1-\Xi(y+x / k)+\Xi(y)-\Xi(z+x / k)+\Xi(z))^{k-2} d \Xi(z) d \Xi(y) \\
& -2 \int_{\mathbb{R}} \int_{y}^{y+x / k}(1-\Xi(y+x / k)+\Xi(y)-\Xi(z+x / k)+\Xi(z))^{k-2} d \Xi(z) d \Xi(y)+O\left(\frac{1}{k}\right) \\
& =\int_{0}^{1} \int_{0}^{1}(1-\Xi(y+x / k)+\Xi(y)-\Xi(z+x / k)+\Xi(z))^{k-2} d \Xi(z) d \Xi(y)+O\left(\frac{1}{k}\right) .
\end{aligned}
$$

Compute

$$
\begin{aligned}
& (1-\Xi(y+x / k)+\Xi(y)-\Xi(z+x / k)+\Xi(z)) \\
& =(1-\Xi(y+x / k)+\Xi(y))(1-\Xi(z+x / k)+\Xi(z)) \\
& \quad-(\Xi(z+x / k)-\Xi(z))(\Xi(y+x / k)-\Xi(y)) \\
& =(1-\Xi(y+x / k)+\Xi(y))(1-\Xi(z+x / k)+\Xi(z))+O\left(k^{-2}\right) .
\end{aligned}
$$

Hence, plugging this into the previous formula, we get

$$
\begin{aligned}
& \mathbb{E}\left(D L S_{\xi}^{2}\left(x, k ; I_{\Lambda}, \omega, \Lambda\right)\right)=\left[\int_{0}^{1}(1-\Xi(y+x / k)+\Xi(y))^{k-2} d \Xi(y)\right]^{2}+O\left(\frac{1}{k}\right) \\
& =\left[\int_{0}^{1}(1-\Xi(y+x / k)+\Xi(y))^{k-1}\left(1+O\left(\frac{1}{k}\right)\right) d \Xi(y)\right]^{2}+O\left(\frac{1}{k}\right) \\
& =D\left(k, E_{0}+I_{\Lambda}, \Lambda\right)^{2}+O\left(\frac{1}{k}\right)
\end{aligned}
$$

This completes the proof of (4.32).

Fix $E_{0}$ such that (1.25) be satisfied and let us prove (4.34). For $I_{\Lambda} \subset J_{\Lambda}$, by Lemma 2.3, for $x \in[0,1]$, we have,

$$
\sup _{y \in[0,1-x / k]}\left|\Xi(y+x / k)-\Xi(y)-\frac{x}{k}\right| \leq \frac{x}{k} \alpha_{k} \quad \text { and } \sup _{y \in[1-x / k, 1]}|\Xi(y+x / k)-\Xi(y)| \leq \frac{x\left(1+\alpha_{k}\right)}{k}
$$

where $\sup _{N_{\Lambda} K_{\Lambda}^{-1} \leq k \leq N_{\Lambda} K_{\Lambda}} \sup _{I_{\Lambda} \subset J_{\Lambda}}\left|\alpha_{k}\right| \rightarrow 0$ as $|\Lambda| \rightarrow+\infty$.

Hence, for $k$ large,

$$
\left|D\left(k, E_{0}+I_{\Lambda}, \Lambda\right)-e^{-x}\right| \leq \int_{0}^{1-x / k}\left|e^{C \alpha_{k}}-1\right| d \Xi(y)+\int_{1-x / k}^{1} e^{C x\left(1+\alpha_{k}\right) / k} d \Xi(y) \leq C\left(\alpha_{k}+\frac{1}{k}\right) .
$$

This completes the proof of (4.34) and, thus, of Lemma 4.3. 
4.3.2. The proof of Theorem 1.4. By a classical result (see e.g. [PS98]), as the functions we are considering are monotonous and as $x \mapsto e^{-x}$ is continuous on $[0,+\infty)$, it suffices to prove the almost sure pointwise convergence of $D L S\left(x ; E_{0}+I_{\Lambda}, \omega, \Lambda\right)$ to $e^{-x}$ (for $\left.x>0\right)$.

Fix $\xi^{\prime}<\xi$. Pick $I_{\Lambda}$ as in Theorem 1.4. We distinguish two cases:

(1) if $I_{\Lambda}$ satisfies (4.26) for $\alpha$ chosen as in section 4.3 .1 (see the discussion in the beginning of that section), we can apply Theorem 1.2 and Lemma 4.3 to $I_{\Lambda}$ itself. We then set $K_{\Lambda}=1$ and $I_{1, \Lambda}=I_{\Lambda}$

(2) if not, as already mentioned, we cover $I_{\Lambda}$ with disjoint intervals $\left(I_{k, \Lambda}\right)_{1 \leq k \leq K_{\Lambda}}$ satisfying (4.26) of such that, for each $I_{k, \Lambda}$, we can apply Theorem 1.2 and Lemma 4.3; as there are at most $o\left(|\Lambda|^{\alpha}\right)$ such intervals (recall that $N$ is the distribution function of an absolutely continuous measure), the probability estimate for the set of good configurations $\mathcal{Z}_{\Lambda}$ (i.e. those for which one has the description given by Theorem 1.2 and Lemma 4.3 for all the intervals $\left.\left(I_{k, \Lambda}\right)_{1 \leq k \leq K_{\Lambda}}\right)$ still satisfies $\mathbb{P}\left(\mathcal{Z}_{\Lambda}\right) \geq 1-O\left(|\Lambda|^{-p}\right)$ for some arbitrary $p>0$.

This yields

$$
\left|D L S\left(x ; E_{0}+I_{\Lambda}, \omega, \Lambda\right)-\sum_{k=1}^{K_{\Lambda}} D L S\left(x ; E_{0}+I_{k, \Lambda}, \omega, \Lambda\right) \frac{N_{\Lambda}\left(E_{0}+I_{k, \Lambda}\right)}{N_{\Lambda}\left(I_{\Lambda}\right)}\right| \leq \frac{K_{\Lambda}}{N_{\Lambda}\left(I_{\Lambda}\right)}
$$

where we recall that $N_{\Lambda}(I)$ is the (random) number of eigenvalues of $H_{\omega}(\Lambda)$ in $I$.

We first study $D L S\left(x ; E_{0}+I_{k, \Lambda}, \omega, \Lambda\right)$.

By (1.24), (1.20) and the approximation of the eigenvalues given by Theorem 1.2 , for $\omega \in \mathcal{Z}_{\Lambda}$, if $J=\#\left\{1 \leq j \leq \tilde{N} ; X_{j}=1\right\}$ then we have

$$
D^{-}\left(\omega, E_{0}+I_{k, \Lambda}, \Lambda\right)+\alpha_{\Lambda} \geq D L S\left(x ; E_{0}+I_{k, \Lambda}, \omega, \Lambda\right) \geq D^{+}\left(\omega, E_{0}+I_{k, \Lambda}, \Lambda\right)-\alpha_{\Lambda}
$$

where

- $D^{ \pm}\left(\omega, E_{0}+I_{k, \Lambda}, \Lambda ; J\right):=\frac{\#\left\{1 \leq j \leq J ; \bar{\xi}_{j+1}-\bar{\xi}_{j} \geq x / N_{k, \Lambda} \pm|\Lambda|^{-2}\right\}}{N_{k, \Lambda}} ;$

- $\left(\xi_{j}\right)_{j}$ are the renormalized eigenvalues defined in (4.30) at the beginning of section 4.3 for the energy interval $E_{0}+I_{k, \Lambda}$;

- $N_{k, \Lambda}=N\left(E_{0}+I_{k, \Lambda}\right)|\Lambda|$ and $\alpha_{\Lambda} \rightarrow 0$ as $|\Lambda| \rightarrow+\infty$.

Define the random variables

$$
D^{ \pm}\left(\omega, E_{0}+I_{k, \Lambda}, \Lambda\right)=D^{ \pm}\left(\omega, E_{0}+I_{k, \Lambda}, \Lambda ; \#\left\{1 \leq j \leq \tilde{N} ; X_{j}=1\right\}\right) \mathbf{1}_{\mathcal{Z}_{\Lambda}} .
$$

We prove

Lemma 4.4. One has

$$
\sup _{1 \leq k \leq K_{\Lambda}}\left[\left(N_{\Lambda}^{k}\right)^{1 / 4} \cdot \mathbb{E}\left(\left|D^{ \pm}\left(\omega, E_{0}+I_{k, \Lambda}, \Lambda\right)-D\left(N_{k, \Lambda}, E_{0}+I_{k, \Lambda}, \Lambda\right)\right|^{2}\right)\right] \leq C .
$$

Before proving Lemma 4.4, let us use it to complete the proof of Theorem 1.4. We first prove the almost sure convergence for a subsequence.

For $\Lambda=\Lambda_{L^{5 / \rho}}$, by the first condition in (1.26), summing the estimate of Lemma 4.4 for $1 \leq k \leq$ $K_{\Lambda_{L^{5 / \rho}}}$, we get that

$$
\sum_{k=1}^{K_{\Lambda} L^{5 / \rho}} \sum_{L \geq 1} \mathbb{E}\left(\left|D^{ \pm}\left(\omega, E_{0}+I_{k, \Lambda_{L^{5 / \rho}}}, \Lambda_{L^{5 / \rho}}\right)-D\left(N_{\Lambda_{L^{5 / \rho}}}, E_{0}+I_{k, \Lambda_{L^{5 / \rho}}}, \Lambda_{L^{5 / \rho}}\right)\right|^{2}\right)<+\infty .
$$

By (4.34) of Lemma 4.3, as $E_{0} \in I$ satisfying (1.25), we know that

$$
\sup _{1 \leq k \leq K_{\Lambda}}\left|D\left(N_{\Lambda}, E_{0}+I_{k, \Lambda}, \Lambda\right)-e^{-x}\right| \underset{|\Lambda| \rightarrow+\infty}{\rightarrow} 0 .
$$


Hence, $\omega$-almost surely,

$$
\sup _{1 \leq k \leq K_{\Lambda_{L}} / \rho}\left|D^{ \pm}\left(\omega, E_{0}+I_{k, \Lambda_{L^{5 / \rho}}}, \Lambda_{L^{5 / \rho}}\right)-e^{-x}\right| \underset{L \rightarrow+\infty}{\rightarrow} 0 .
$$

Thus, by (4.37), $\omega$-almost surely

$$
\sup _{1 \leq k \leq K_{\Lambda} L^{5 / \rho}}\left|D L S\left(x ; E_{0}+I_{k, \Lambda}, \omega, \Lambda\right)-e^{-x}\right| \underset{L \rightarrow+\infty}{\rightarrow} 0 .
$$

Plugging this into (4.36), we obtain that, $\omega$-almost surely

$$
D L S\left(x ; E_{0}+I_{\Lambda_{L^{5 / \rho}}}, \omega, \Lambda_{L^{5 / \rho}}\right) \underset{L \rightarrow+\infty}{\rightarrow} e^{-x} .
$$

To derive the almost sure convergence of $\left(D L S\left(x ; E_{0}+I_{\Lambda}, \omega, \Lambda\right)\right)_{\Lambda}$, we use

Lemma 4.5. Fix $p>0$ and $r>0$. Let $\left(I_{\Lambda}\right)_{\Lambda}$ be as Theorem 1.4 or $I_{\Lambda}=J, J$ as in Theorem 1.6. Recall that $N_{\Lambda_{L}}=N_{\Lambda_{L}}\left(I_{L}\right)$.

Then, $\omega$-almost surely, for $L$ sufficiently large, if $L^{\prime} \in\left[L^{p},(L+1)^{p}\right]$, except for at most o $\left(N_{\Lambda_{L^{p}}}\right)$ of them, all the eigenvalues of $H_{\omega}\left(\Lambda_{L^{\prime}}\right)$ in $E_{0}+I_{\Lambda_{L^{\prime}}}$ are at a distance less than $L^{-r}$ to an eigenvalue of $H_{\omega}\left(\Lambda_{L^{p}}\right)$ in $E_{0}+I_{\Lambda_{L^{p}}}$.

Pick $p=5 / \rho$ and $r>d$. For $L^{p} \leq L^{\prime} \leq(L+1)^{p}$, by assumption (1.26) on $I_{\Lambda}$, one has $N\left(E_{0}+\right.$ $\left.I_{\Lambda_{L^{\prime}}}\right)=N\left(E_{0}+I_{\Lambda_{L} p}\right)(1+o(1)) ;$ so $N_{\Lambda_{L^{\prime}}}=N_{\Lambda_{L} p}(1+o(1))$. Lemma 4.5 then implies that, $\omega$-almost surely, for $L^{p} \leq L^{\prime} \leq(L+1)^{p}$ and $L$ sufficiently large, one has

$$
D L S\left(x ; I_{\Lambda_{L^{\prime}}}, \omega, \Lambda_{L^{\prime}}\right)=D L S\left(x ; I_{\Lambda_{L^{p}}}, \omega, \Lambda_{L^{p}}\right)+o(1) .
$$

Hence, $\omega$-almost surely, $D L S\left(x ; I_{\Lambda}, \omega, \Lambda\right)$ converges to $e^{-x}$ when $|\Lambda| \rightarrow+\infty$. This completes the proof of Theorem 1.4.

Remark 4.3. If one does not assume one of the conditions in (1.26), the estimate (4.38) and Lemma 4.5 are not sufficient anymore to obtain the almost sure convergence for $D L S\left(x ; I_{\Lambda}, \omega, \Lambda\right)$. Nevertheless, plugging the estimates (4.39), (4.38) and (4.37) into (4.36), we see that

$$
\mathbb{E}\left(\left|D L S\left(x ; I_{\Lambda}, \omega, \Lambda\right)-e^{-x}\right|\right) \underset{|\Lambda| \rightarrow+\infty}{\rightarrow} 0 .
$$

This guarantees the convergence in probability.

Proof of Lemma 4.4. We now fix $1 \leq k \leq K_{\Lambda}$ and, to alleviate the notations, we will write $I_{\Lambda}:=I_{k, \Lambda}$ and $N_{\Lambda}=N_{k, \Lambda}=N\left(I_{\Lambda}\right)|\Lambda|$. The interval $I_{\Lambda}$ satisfies the requirements to apply Theorem 1.2 and Lemma 4.3 (see the discussion in the beginning of section 4.3.1).

As $D^{ \pm}$is bounded by $C|\Lambda|$, as the $\left(X_{j}\right)_{j}$ are i.i.d. and as $\mathbb{P}\left(\mathcal{Z}_{\Lambda}\right) \leq|\Lambda|^{-p}$ for any $p>0$, we compute

$$
\begin{aligned}
\mathbb{E}\left(\left|D^{ \pm}\left(\omega, I_{\Lambda}, \Lambda\right)-D\left(N_{\Lambda}, E_{0}+I_{\Lambda}, \Lambda\right)\right|^{2}\right) & \\
\leq \frac{1}{|\Lambda|^{p}}+ & \sum_{k=0}^{\tilde{N}}\left(\begin{array}{c}
\tilde{N} \\
k
\end{array}\right) \mathbb{P}\left(X_{1}=1\right)^{k}\left(1-\mathbb{P}\left(X_{1}=1\right)\right)^{\tilde{N}-k} . \\
& \cdot \mathbb{E}\left(\left|D L S_{\xi}\left(x \pm|\Lambda|^{-2}, k ; E_{0}+I_{\Lambda}, \omega, \Lambda\right)-D\left(N_{\Lambda}, E_{0}+I_{\Lambda}, \Lambda\right)\right|^{2}\right) .
\end{aligned}
$$

Recall that $N_{\Lambda}=N\left(I_{\Lambda}\right)|\Lambda|$. As $\mathbb{P}\left(X_{1}=1\right)=N\left(I_{\Lambda}\right) \ell_{\Lambda}^{d}(1+o(1))$ and $\tilde{N}=|\Lambda| \ell_{\Lambda}^{-d}(1+o(1))$, for any $\varepsilon>0$, there exists $\delta>0$ such that

$$
\sum_{\left|k-N_{\Lambda}\right| \geq N_{\Lambda}^{1 / 2+\varepsilon}}\left(\begin{array}{c}
\tilde{N} \\
k
\end{array}\right) \mathbb{P}\left(X_{1}=1\right)^{k}\left(1-\mathbb{P}\left(X_{1}=1\right)\right)^{\tilde{N}-k} \leq e^{-N_{\Lambda}^{\delta}} .
$$


Hence, by the first condition in (1.26), one gets

$$
\begin{aligned}
\mathbb{E}\left(\left|D^{ \pm}\left(\omega, I_{\Lambda}, \Lambda\right)-D\left(N_{\Lambda}, E_{0}+I_{\Lambda}, \Lambda\right)\right|^{2}\right) & \\
\leq & \frac{1}{|\Lambda|^{p}}+e^{-N_{\Lambda}^{\delta} / 2}+2 \sum_{\left|k-N_{\Lambda}\right|<N_{\Lambda}^{1 / 2+\varepsilon}}\left|D\left(k, E_{0}, \Lambda\right)-D\left(N_{\Lambda}, E_{0}+I_{\Lambda}, \Lambda\right)\right|^{2} \\
& +2 \sum_{\left|k-N_{\Lambda}\right|<N_{\Lambda}^{1 / 2+\varepsilon}} \mathbb{E}\left(\left|D L S_{\xi}\left(x \pm|\Lambda|^{-2}, k ; E_{0}+I_{\Lambda}, \omega, \Lambda\right)-D\left(k, E_{0}, \Lambda\right)\right|^{2}\right) .
\end{aligned}
$$

For $\left|k-N_{\Lambda}\right|<N_{\Lambda}^{1 / 2+\varepsilon}$, let us estimate $D\left(k, E_{0}, \Lambda\right)-D\left(N_{\Lambda}, E_{0}+I_{\Lambda}, \Lambda\right)$ that is

$$
\begin{aligned}
D\left(k, E_{0}, \Lambda\right) & -D\left(N_{\Lambda}, E_{0}+I_{\Lambda}, \Lambda\right) \\
& =\int_{0}^{1}\left((1-\Xi(y+x / k)+\Xi(y))^{k-1}-\left(1-\Xi\left(y+x / N_{\Lambda}\right)+\Xi(y)\right)^{N_{\Lambda}-1}\right) d \Xi(y) .
\end{aligned}
$$

Using (4.35), for $\left|k-N_{\Lambda}\right|<N_{\Lambda}^{1 / 2+\varepsilon}$, compute

$$
\begin{aligned}
& \left|(1-\Xi(y+x / k)+\Xi(y))^{k-1}-\left(1-\Xi\left(y+x / N_{\Lambda}\right)+\Xi(y)\right)^{N_{\Lambda}-1}\right| \\
& =(1-\Xi(y+x / k)+\Xi(y))^{k-1} . \\
& \quad \cdot\left|1-\left(\frac{1-\Xi\left(y+x / N_{\Lambda}\right)+\Xi(y)}{1-\Xi(y+x / k)+\Xi(y)}\right)^{N_{\Lambda}}\left(1-\Xi\left(y+x / N_{\Lambda}\right)+\Xi(y)\right)^{N_{\Lambda}-k}\right| \\
& \leq C\left(1-e^{-C\left(N_{\Lambda}-k\right) / N_{\Lambda}} e^{-C\left(N_{\Lambda}-k\right) / k}\right) \leq C N_{\Lambda}^{-1 / 2+\varepsilon} .
\end{aligned}
$$

Hence, plugging this into (4.43), one obtains

$$
\left|D\left(k, E_{0}, \Lambda\right)-D\left(N_{\Lambda}, E_{0}+I_{\Lambda}, \Lambda\right)\right| \leq C N_{\Lambda}^{-1 / 2+\varepsilon} .
$$

Plugging this and the result of Lemma 4.3 into (4.42), we obtain

$$
\mathbb{E}\left(\left|D^{ \pm}\left(\omega, I_{\Lambda}, \Lambda\right)-D\left(N_{\Lambda}, E_{0}+I_{\Lambda}, \Lambda\right)\right|^{2}\right) \leq C N_{\Lambda}^{-1 / 2+3 \varepsilon} .
$$

We pick $\varepsilon=1 / 12$ to completes the proof of Lemma 4.4.

Proof of Lemma 4.5. Pick $\alpha \in(0,1)$ and $q>1$. By Lemma 3.1, with a probability at least $1-L^{-q}$, the eigenvalues of $H_{\omega}\left(\Lambda_{L^{\prime}}\right)$ in $E_{0}+I_{\Lambda_{L^{\prime}}}$ that are at a distance more than $L^{-r}$ to an eigenvalue of $H_{\omega}\left(\Lambda_{L^{p}}\right)$ in $E_{0}+I_{\Lambda_{L^{p}}}$ fall into two categories:

(1) either they belong to $E_{0}+\left(I_{\Lambda_{L^{p}}} \backslash I_{\Lambda_{L^{\prime}}}\right)$ which may be empty,

(2) or they have a localization center that belongs e.g. to the annulus $\Lambda_{L^{\prime}} \backslash \Lambda_{L^{p}-L^{\alpha}}$.

The numbers of eigenvalues in the first category is bounded by $o\left(N_{\Lambda_{L^{p}}}\right)$ as a consequence of the second part of assumption (1.26). The numbers of eigenvalues in the second category is bounded by $o\left(N_{\Lambda_{L} p}\right)$. The Borel-Cantelli lemma then implies Lemma 4.5.

4.3.3. The proof of Theorem 1.6. The proof is analogous to that of Theorem 1.4. As in that proof, it suffices to prove the almost sure pointwise convergence of $D L S^{\prime}(x ; J, \omega, \Lambda)$ to $g_{\nu, J}(x)$ for $x>0$. Fix $\xi^{\prime}<\xi$. Pick $J=[a, b]$ as in Theorem 1.6. We can then cover it with disjoint intervals $\left(I_{j, \Lambda}\right)_{1 \leq j \leq J_{\Lambda}}$ of length $|J||\Lambda|^{-\alpha}$ (here $\alpha$ is chosen as in the beginning of section 4.3.1) and containing the point $e_{j, \Lambda}:=a+(j-1 / 2)|J||\Lambda|^{-\alpha}$ (so that $J_{\Lambda} \asymp|\Lambda|^{\alpha}$ ) and such that, for each $I_{j, \Lambda}$, we can apply Lemma 4.3. This yields

$$
\left|D L S^{\prime}(x ; J, \omega, \Lambda)-\sum_{j=1}^{J_{\Lambda}} D L S\left(\nu\left(e_{j, \Lambda}\right) x / N(J) ; I_{j, \Lambda}, \omega, \Lambda\right) \frac{N_{\Lambda}\left(I_{j, \Lambda}\right)}{N_{\Lambda}(J)}\right| \leq \frac{C J_{\Lambda}}{|\Lambda|}
$$


where $D L S^{\prime}$ is defined in (1.30).

Using the uniform continuity of $\nu$, the same computations as in the proof of Theorem 1.4 yield the following analogue of (4.40): for all $\varepsilon>0$,

$$
\sup _{\substack{1 \leq j \leq J_{\Lambda} \\ \nu\left(e_{j, \Lambda}\right) \geq \varepsilon \\ \Lambda=\Lambda_{L^{5 / \rho}}}}\left|D L S\left(\nu\left(e_{j, \Lambda}\right) x / N(J) ; I_{j, \Lambda}, \omega, \Lambda\right)-e^{-\nu\left(e_{j, \Lambda}\right) x / N(J)}\right| \underset{L \rightarrow+\infty}{\rightarrow} 0, \quad \omega-\text { a.s.. }
$$

The large deviation principle for the eigenvalue counting function, Theorem 1.3, ensures that, $\omega$ almost surely, for $|\Lambda|$ large, $N_{\Lambda}(J) \geq|\Lambda||J| / 2$ and

$$
\lim _{L \rightarrow+\infty} \sup _{\substack{1 \leq j \leq J_{\Lambda} \\ \nu\left(e_{j, \Lambda}\right) \geq \varepsilon}}\left|\frac{N_{\Lambda}\left(I_{j, \Lambda}\right)}{N_{\Lambda}(J)} \cdot \frac{N(J)}{\nu\left(e_{j, \Lambda}\right)\left|I_{j, \Lambda}\right|}-1\right|=0 .
$$

Moreover, using the uniform continuity of $\nu$ on $J$, we have that for any $\delta>0$, there exists $\varepsilon>0$ such that, for $L$ sufficiently large, one has

$$
\sup _{\substack{1 \leq j \leq J_{\Lambda} \\ \nu\left(e_{j, \Lambda}\right) \leq \varepsilon}}\left|\frac{N_{\Lambda}\left(I_{j, \Lambda}\right)}{\left|I_{j, \Lambda}\right| N_{\Lambda}(J)}\right| \leq \delta .
$$

Hence, by (4.44), $\omega$-almost surely, there exists $C>0$ such that $\varepsilon>0$, one has

$$
\sum_{\substack{1 \leq j \leq J_{\Lambda} \\ \nu\left(e_{j}, \Lambda\right) \leq \varepsilon \\ \Lambda=\Lambda_{L^{5 / \rho}}}} D L S\left(\nu\left(e_{j, \Lambda}\right) x / N(J) ; I_{j, \Lambda}, \omega, \Lambda\right) \frac{N_{\Lambda}\left(I_{j, \Lambda}\right)}{N_{\Lambda}(J)} \leq \delta \sum_{\substack{1 \leq j \leq J_{\Lambda} \\ \nu\left(e_{j, \Lambda}\right) \leq \varepsilon \\ \Lambda=\Lambda_{L^{5 / \rho}}}}\left|I_{j, \Lambda}\right| \leq C \delta,
$$

thus

$$
\limsup _{\substack{L \rightarrow+\infty \\ \Lambda=\Lambda} L^{5 / \rho}}\left|D L S^{\prime}(x ; J, \omega, \Lambda)-\sum_{\substack{1 \leq j \leq J_{\Lambda} \\ \nu\left(e_{j, \Lambda}\right) \geq \varepsilon \\ \Lambda=\Lambda_{L^{5 / \rho}}}} \frac{\nu\left(e_{j, \Lambda}\right)\left|I_{j, \Lambda}\right|}{N(J)} e^{-\nu\left(e_{j, \Lambda}\right) x}\right| \leq C \delta .
$$

On the other hand, as $\nu$ is continuous, non negative on $J$ and $x>0$, one has, for any $\delta>0$, taking $\varepsilon>0$ sufficiently small, one has

$$
\left|\sum_{\substack{1 \leq j \leq J_{\Lambda} \\ \nu\left(e_{j, \Lambda}\right)<\varepsilon}} \frac{\nu\left(e_{j, \Lambda}\right)\left|I_{j, \Lambda}\right|}{N(J)} e^{-\nu\left(e_{j, \Lambda}\right) x / N(J)}\right| \leq \delta
$$

and

$$
\begin{aligned}
\lim _{L \rightarrow+\infty} \sum_{\substack{1 \leq j \leq J_{\Lambda} \\
\Lambda=\Lambda}} \frac{\nu\left(e_{j, \Lambda}\right)\left|I_{j, \Lambda}\right|}{N(J)} e^{-\nu\left(e_{j, \Lambda}\right) x / N(J)} & =\frac{|J|}{N(J)} \int_{0}^{1} e^{-\nu(a+|J| y) x / N(J)} \nu(a+|J| y) d y \\
& =\frac{1}{N(J)} \int_{J} e^{-\nu(\lambda) x / N(J)} \nu(\lambda) d \lambda .
\end{aligned}
$$

Thus, for $\delta>0$, we get

$$
\limsup _{\substack{L \rightarrow+\infty \\ \Lambda=\Lambda_{L^{5 / \rho}}}}\left|D L S^{\prime}(x ; J, \omega, \Lambda)-\int_{J} e^{-\nu_{J}(\lambda) x} \nu_{J}(\lambda) d \lambda\right| \leq \delta .
$$


Hence, letting $\delta$ tend to 0 , we get that, $\omega$ almost surely,

$$
\lim _{\substack{L \rightarrow+\infty \\ \Lambda=\Lambda_{L^{5 / \rho}}}} D L S^{\prime}(x ; J, \omega, \Lambda)=\int_{J} e^{-\nu_{J}(\lambda) x} \nu_{J}(\lambda) d \lambda .
$$

To complete the proof of Theorem 1.6, we use Lemma 4.5 as in the proof of Theorem 1.4.

4.4. Study of the localization centers statistics. We prove Theorems 1.7.

As in the proofs of Theorems 1.4 and 1.6, it suffices to prove the simple convergence in (1.34) to obtain the uniform convergence as we are dealing with non increasing functions and the limit is continuous. Thus, pick $s>0$ and to prove (1.34), it suffices to prove

$$
\mathbb{E}\left(\left|D C S\left(s ; I_{\Lambda}, \Lambda, \omega\right)-e^{-s^{d}}\right|^{2}\right) \underset{\Lambda \nearrow \mathbb{R}^{d}}{\rightarrow} 0 .
$$

We apply Theorem 1.2 to the cube $\Lambda$ and the interval $I_{\Lambda}$ satisfying (1.33). We keep the same notations as in Theorem 1.2. Let $\Gamma$ denote the set of centers $\gamma_{i}$ constructed in Theorem 1.2. Let $\Gamma_{b}$ denote the set of $\gamma \in \Gamma$ that do not not satisfy (1), (2) and (3) of Theorem 1.1. Theorem 1.2 states that, for $\omega \in \mathcal{Z}_{\Lambda}$, one has

$$
\# \Gamma_{b}=|\Lambda| \ell^{-d} O\left[\left(\left|I_{\Lambda}\right| \ell^{d}\right)^{1+\rho}+N\left(E_{0}+I_{\Lambda}\right) \ell^{d-1} \ell^{\prime}\right]
$$

where $\ell$ and $\ell^{\prime}$ are defined in Theorem 1.2.

By (1.33), one has

$$
N\left(E_{0}+I_{\Lambda}\right)^{-1 / d} \gg \ell
$$

Thus, if we define

$$
\Gamma^{\prime}=\left\{\gamma \in \Gamma ; \operatorname{dist}\left(\gamma, \Gamma_{b}\right) \geq 10 s\left(N\left(E_{0}+I_{\Lambda}\right)\right)^{-1 / d}\right\}
$$

then

$$
\# \Gamma^{\prime}=O\left(\# \Gamma_{b} \cdot N\left(E_{0}+I_{\Lambda}\right)^{-1} \ell^{-d}\right)=o\left(|\Lambda| \ell^{-d}\right) .
$$

For $\gamma \in \Gamma$, define the random variable $X_{\gamma}$ to be equal

- to 1 if $H_{\omega}\left(\Lambda_{\ell}(\gamma)\right)$ has an eigenvalue in $I_{\Lambda}$, and, for all $\gamma^{\prime} \in \Gamma$ such that $\left|\gamma^{\prime}-\gamma\right| \leq\left(N\left(E_{0}+\right.\right.$ $\left.\left.I_{\Lambda}\right)\right)^{-1 / d} s, H_{\omega}\left(\Lambda_{\ell}(\gamma)\right)$ has no eigenvalue in $I_{\Lambda}$,

- to 0 if not.

Then, by (4.46), (4.47) and the estimate given in Theorem 1.2 on the number of eigenvalues of $H_{\omega}(\Lambda)$ not associated to an eigenvalue for a cube $\left(\Lambda_{\ell}(\gamma)\right)_{\gamma \in \Gamma}$, we get that, for any $\varepsilon>0$, for $|\Lambda|$ sufficiently large, one has

$$
\begin{aligned}
\sup _{\omega \in \mathcal{Z}_{\Lambda}}\left(D C S^{\prime}\left(s-\varepsilon ; I_{\Lambda}, \Lambda, \omega\right)-D\right. & \left.C S\left(s ; I_{\Lambda}, \Lambda, \omega\right)\right) \\
& +\sup _{\omega \in \mathcal{Z}_{\Lambda}}\left(D C S\left(s ; I_{\Lambda}, \Lambda, \omega\right)-D C S^{\prime}\left(s+\varepsilon ; I_{\Lambda}, \Lambda, \omega\right)\right) \leq \varepsilon
\end{aligned}
$$

where $\operatorname{DCS}\left(s ; I_{\Lambda}, \Lambda, \omega\right)$ is defined in $(1.32)$ and

$$
D C S^{\prime}\left(s ; I_{\Lambda}, \Lambda, \omega\right)=\frac{1}{N\left(E_{0}+I_{\Lambda}, \Lambda, \omega\right)} \sum_{\gamma \in \Gamma} X_{\gamma} .
$$

As $0 \leq D C S\left(s ; I_{\Lambda}, \Lambda, \omega\right) \leq 1$ and $0 \leq D C S^{\prime}\left(s ; I_{\Lambda}, \Lambda, \omega\right) \leq 2$ (for $\Lambda$ large), in view of (4.48), to prove (4.45), it suffices to prove

$$
\mathbb{E}\left(\left|D C S^{\prime}\left(s ; I_{\Lambda}, \Lambda, \omega\right)-e^{-s^{d}}\right|^{2}\right) \underset{\Lambda \nearrow \mathbb{R}^{d}}{\rightarrow} 0 .
$$


As the Hamiltonians $H_{\omega}\left(\Lambda_{\ell}(\gamma)\right)$ and $H_{\omega}\left(\Lambda_{\ell}(\gamma)\right)$ are independent when $\gamma \neq \gamma^{\prime},\left(\gamma, \gamma^{\prime}\right) \in \Gamma^{2}$, using Lemma 2.1, we compute

$$
\begin{aligned}
\mathbb{E}\left(X_{\gamma}\right) & =\left(1-N\left(E_{0}+I_{\Lambda}\right) \ell^{d}\right)^{\left(N\left(E_{0}+I_{\Lambda}\right) \ell^{d}\right)^{-1} s^{d}-1} N\left(E_{0}+I_{\Lambda}\right) \ell^{d}+o\left(\left|I_{\Lambda}\right| \ell^{d}\right) \\
& =\mathrm{e}^{-s^{d}} N\left(E_{0}+I_{\Lambda}\right) \ell^{d}+o\left(\left|I_{\Lambda}\right| \ell^{d}\right) .
\end{aligned}
$$

Thus, using Theorem 1.3, one computes

$$
\begin{aligned}
\mathbb{E}\left(D C S^{\prime}\left(s ; I_{\Lambda}, \Lambda, \omega\right)\right) & =\mathbb{E}\left(\frac{1}{N\left(E_{0}+I_{\Lambda}, \Lambda, \omega\right)} \sum_{\gamma \in \Gamma} X_{\gamma}\right) \\
& =\frac{1}{N\left(E_{0}+I_{\Lambda}\right)|\Lambda|} \frac{|\Lambda|}{\ell^{d}} \mathrm{e}^{-s^{d}} N\left(E_{0}+I_{\Lambda}\right) \ell^{d}+o(1)=\mathrm{e}^{-s^{d}}+o(1) .
\end{aligned}
$$

On the other hand, by (4.46) and their definition, $X_{\gamma}$ and $X_{\gamma^{\prime}}$ are independent when $\left|\gamma^{\prime}-\gamma\right| \leq$ $2\left(N\left(E_{0}+I_{\Lambda}\right)\right)^{-1} s+1$. Hence, using Theorem 1.3, one computes

$$
\begin{aligned}
& \Delta V:=\mathbb{E}\left(D C S^{\prime}\left(s ; I_{\Lambda}, \Lambda, \omega\right)^{2}\right)-\mathbb{E}\left(D C S^{\prime}\left(s ; I_{\Lambda}, \Lambda, \omega\right)\right)^{2} \\
&=\frac{1+o(1)}{\left(N\left(E_{0}+I_{\Lambda}\right)|\Lambda|\right)^{2}} \sum_{\gamma \in \Gamma} \sum_{\substack{\gamma^{\prime} \in \Gamma \\
\left|\gamma^{\prime}-\gamma\right| \leq 2\left(N\left(E_{0}+I_{\Lambda}\right)\right)^{-1} s+1}}\left(\mathbb{E}\left(X_{\gamma} X_{\gamma^{\prime}}\right)-\mathbb{E}\left(X_{\gamma}\right) \mathbb{E}\left(X_{\gamma^{\prime}}\right)\right) .
\end{aligned}
$$

Thus, as $\# \Gamma \leq C|\Lambda| / \ell^{d}$, by Lemma 2.1 , one has

$$
\Delta V \leq \frac{2}{\left(N\left(E_{0}+I_{\Lambda}\right)|\Lambda|\right)^{2}} \frac{|\Lambda|}{\ell^{d}} \frac{1}{\ell^{d} N\left(E_{0}+I_{\Lambda}\right)} N\left(E_{0}+I_{\Lambda}\right) \ell^{d}=\frac{2}{N^{2}\left(E_{0}+I_{\Lambda}\right)|\Lambda| \ell^{d}} .
$$

Condition (1.33) then ensures that the choice of the length $\ell$ in Theorem 1.2 can be made such that $N^{2}\left(E_{0}+I_{\Lambda}\right)|\Lambda| \ell^{d} \rightarrow+\infty$ as $|\Lambda| \rightarrow+\infty$.

Thus, (4.50) and (4.51) imply (4.49). This completes the proof of Theorem 1.7.

\section{Proof of Proposition 1.2 And Theorem 1.8}

We start with the proof of Proposition 1.2 then we prove Theorem 1.8.

5.1. The proof of Proposition 1.2. Let us prove point (1). Consider $x(E)$ and $x^{\prime}(E)$ two centers of localization for some energy $E \in I$. Let $\varphi$ be a normalized eigenstate associated to $E$. Then, by the assumption (Loc'), we know that, for all $x$,

$$
\|\varphi\|_{x}^{2} \leq C_{\omega}\langle x(E)\rangle^{q} e^{-\gamma|x-x(E)|^{\xi}}\left\langle x^{\prime}(E)\right\rangle^{q} e^{-\gamma\left|x-x^{\prime}(E)\right|^{\xi}} .
$$

Hence, summing over $x$, we get that

$$
1 \leq C_{d} C_{\omega}\langle x(E)\rangle^{q}\left\langle x^{\prime}(E)\right\rangle^{q} \frac{1}{\gamma^{d / \xi}} e^{-\gamma\left|x(E)-x^{\prime}(E)\right|^{\xi}} .
$$

Taking the logarithm, we immediately get (1.36).

Let us now prove point (2). First, note that, by the Wegner estimate (W), the condition $N\left(I_{L}\right) L^{d} \rightarrow$ $+\infty$ implies that $\left|I_{L}\right| \gtrsim L^{-d}$ for $L$ large. Hence, if $J$ is an interval centered at 0 such that $|J|=o\left(L^{-d}\right)$, then $N\left(I_{L}+J\right)=N\left(I_{L}\right)(1+o(1))$.

By definition, one has

$$
N\left(I_{L}, L\right)=\sum_{\substack{n ; E_{n} \in I_{L} \\ \gamma_{n} \in \Lambda_{L}}}\left\|\varphi_{n}\right\|^{2} .
$$

where, for $n, E_{n}$ is an eigenvalues of $H_{\omega}, \varphi_{n}$ an associated eigenfunction and $\gamma_{n}$ a localization center of $\varphi_{n}$ in $\Lambda_{L}$. 
Fix $\alpha \in(0,1)$. Using the estimate (1.35) in the same way as the estimates (Loc) was used in the proof of Lemma 3.1, we get that

$$
\left|N\left(I_{L}, L\right)-\sum_{\substack{n ; E_{n} \in I_{L} \\ \gamma_{n} \in \Lambda_{L}}}\left\|\varphi_{n}\right\|_{\Lambda_{L+L^{\alpha}}}^{2}\right| \leq C_{\omega} L^{q+d} e^{-2 \gamma L^{\alpha \xi}}
$$

where $\|\cdot\|_{\Lambda_{L+L^{\alpha}}}$ denotes the $L^{2}$-norm on the cube $\Lambda_{L+L^{\alpha}}$.

Hence, we have

$$
\begin{aligned}
N\left(I_{L}, L\right) & \leq \sum_{\substack{n ; E_{n} \in I_{L} \\
\gamma \in \Lambda_{L}}}\left\|\varphi_{n}\right\|_{\Lambda_{L+L^{\alpha}}}^{2}+C_{\omega} L^{q+d} e^{-2 \gamma L^{\alpha \xi}} \\
& \leq \operatorname{tr}\left(\mathbf{1}_{I_{L}}\left(H_{\omega}\right) \mathbf{1}_{\Lambda_{L+L^{\alpha}}}\right)+C_{\omega} L^{q+d} e^{-2 \gamma L^{\alpha \xi}} .
\end{aligned}
$$

By standard estimates on Schrödinger operators (see e.g. [Sim05]), we know that

$$
0 \leq \operatorname{tr}\left(\mathbf{1}_{I_{L}}\left(H_{\omega}\right) \mathbf{1}_{\Lambda_{L+L^{\alpha}}}\right) \leq C L^{d}
$$

Pick $\chi$ a smooth cut-off function with gradient vanishing outside $\Lambda_{L+L^{\alpha}} \backslash \Lambda_{L+L^{\alpha} / 2}$. Then, using the localization estimate (Loc'), one easily checks that, for $L$ sufficiently large

- for $n$ such that $E_{n} \in I_{L}$ and $\gamma_{n} \in \Lambda_{L}, \chi \varphi_{n}$ satisfies

$$
\left\|\left(H_{\omega}\left(\Lambda_{L+L^{\alpha}}\right)-E_{n}\right)\left(\chi \varphi_{n}\right)\right\| \leq e^{-L^{\alpha \xi} / C} ;
$$

- the Gram matrix for the family $\left(\chi \varphi_{n}\right)_{E_{n} \in I_{L}, \gamma_{n} \in \Lambda_{L}}$ satisfies

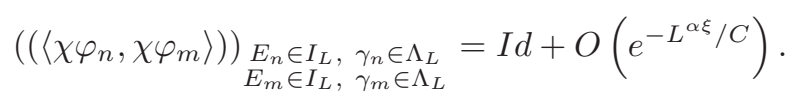

As (5.1) implies that the number of $n$ such that $E_{n} \in I_{L}$ and $\gamma_{n} \in \Lambda_{L}$ is bounded by $C L^{d}$, we get that

$$
N\left(I_{L}, L\right) \leq \operatorname{tr}\left(\mathbf{1}_{\tilde{I}_{L}}\left(H_{\omega}\left(\Lambda_{L+2 L^{\alpha}}\right)\right)\right)+C_{\omega} L^{q+d} e^{-2 \gamma L^{\alpha \xi}}
$$

where $\tilde{I}_{L}=I_{L}+\left[-e^{L^{\alpha} \xi / C}, e^{L^{\alpha} \xi / C}\right]$.

Hence, if, as in section $3, N\left(\omega, \Lambda_{L+L^{\alpha}},, I_{L}\right)$ denotes the number of eigenvalues of $H_{\omega}\left(\Lambda_{L+L^{\alpha}}\right)$ in $I_{L}$, we have

$$
N\left(I_{L}, L\right) \leq N\left(\omega, \Lambda_{L+L^{\alpha}}, I_{L}\right)+C_{\omega} L^{q+d} e^{-2 \gamma L^{\alpha \xi}} .
$$

Recall that we assumed $N\left(I_{L}\right)\left|I_{L}\right|^{-1-\rho} \rightarrow+\infty$ where $\rho$ is given by $(\mathrm{M})$. The proof of Theorem 1.3 then also shows that, if $N\left(\omega, \Lambda_{L+L^{\alpha}}, \Lambda_{L}, I_{L}\right)$ denotes the number of eigenvalues of $H_{\omega}\left(\Lambda_{L+L^{\alpha}}\right)$ in $I_{L}$ having a localization center in $\Lambda_{L}$, then, for any $q$,

$$
\begin{aligned}
\mathbb{P}\left\{\left|N\left(\omega, \Lambda_{L+L^{\alpha}}, \Lambda_{L}, I_{L}\right)-N\left(I_{\Lambda}\right)\right| \Lambda||=o\left(N\left(I_{\Lambda}\right)|\Lambda|\right)\right\} & \geq 1-C_{q}\left(N\left(I_{\Lambda}\right)|\Lambda|\right)^{-q} \\
& \geq 1-C_{q} L^{-2}
\end{aligned}
$$

as $N\left(I_{L}\right) L^{d-\varepsilon} \rightarrow+\infty$ for some $\varepsilon>0$.

So, by the Borel-Cantelli Lemma and Theorem 1.3, we know that, $\omega$-almost surely,

$$
N\left(\omega, \Lambda_{L+L^{\alpha}}, \Lambda_{L}, I_{L}\right)=N\left(\omega, \Lambda_{L+L^{\alpha}},, I_{L}\right)(1+o(1))=N\left(I_{L}\right) L^{d}(1+o(1)) .
$$

One has

$$
N\left(I_{L}, L\right) \geq \sum_{\substack{n ; E_{n} \in I_{L} \\ \gamma_{n} \in \Lambda_{L}}}\left\|\varphi_{n}\right\|_{\Lambda_{L+L^{\alpha}}}^{2}
$$

Using the same cut-off of the eigenfunctions as above, we then see that

$$
N\left(I_{L}, L\right) \geq N\left(\omega, \Lambda_{L+L^{\alpha}}, \Lambda_{L}, I_{L}\right)
$$


Using this estimate, (5.5), (5.4) and Theorem 1.3, we get (1.37) and complete the proof of Proposition 1.2 .

5.2. The proof of Theorem 1.8. Let us now consider consider $\omega$ in the full measure set where the statements of Theorems 1.4, 1.6 and Proposition 1.2 hold. As in section 5.1, $\omega$-almost surely, for $L$ large, if $E$ is an eigenvalue of $H_{\omega}$ with localization center in $\Lambda_{L}$, then there exists $E^{\prime}$ an eigenvalue of $H_{\omega}\left(\Lambda_{L+L^{\alpha}}\right)$ such that $\left|E-E^{\prime}\right| \leq e^{-L^{\alpha} / C}$. To avoid confusion, rename $D L S\left(x ; I_{L}, \omega, L\right)$ defined by (1.24) to $D L S^{f}\left(x ; I_{L}, \omega, L\right)$. Hence, for $\varepsilon>0$ fixed, we have, for $L$ large enough,

- when $\left|I_{L}\right| \rightarrow 0$ satisfying the assumptions of Theorem 1.8, we have

$$
\begin{aligned}
D L S\left(\nu\left(E_{0}\right) x-\varepsilon ; I_{L}, \omega, L\right) & \geq \frac{N\left(I_{L}, L\right)}{N\left(I_{L}, \omega, \Lambda_{L}\right)} \cdot D L S^{f}\left(x ; I_{L}, \omega, L\right) \\
& \geq D L S\left(\nu\left(E_{0}\right) x+\varepsilon ; I_{L}, \omega, L\right) ;
\end{aligned}
$$

- when $I_{L}=J$ a fixed interval satisfying the assumptions of Theorem 1.8, we have

$$
D L S^{\prime}(x-\varepsilon ; J, \omega, L) \geq \frac{N\left(I_{L}, L\right)}{N\left(J, \omega, \Lambda_{L}\right)} D L S^{f}(x ; J, \omega, L) \geq D L S^{\prime}(x+\varepsilon ; J, \omega, L) .
$$

Thus, Theorem 1.8 is an immediate corollary of Theorems 1.4 and 1.6, and the second point of Proposition 1.2.

\section{ApPEndix}

6.1. The proof of Lemma 1.3. Compute

$$
\begin{aligned}
\int_{\mathbb{R}}\left|\frac{N(E+x)-N(E+y)}{x-y}-\nu(E)\right| & =\int_{\mathbb{R}}\left|\frac{1}{x-y} \int_{E+y}^{E+x}(\nu(e)-\nu(E)) d e\right| \\
& \leq \int_{0}^{1}\left(\int_{\mathbb{R}}|\nu(E+y+(x-y) e)-\nu(E)| d E\right) d e .
\end{aligned}
$$

As $\nu \in L^{1}(\mathbb{R})$, Lebesgue's dominated convergence theorem ensures that the last integral converges to 0 when $x$ and $y$ tend to 0 . Hence, the quotient in the first integral converges to 0 for almost every $E$. This completes the proof of Lemma 1.3.

6.2. Local control on the exponential decay of eigenfunctions. In this section, we establish SUDEC and SULE estimates for the eigenfunctions assciated to an eigenvalue in the localized regime of a random operator restricted to a finite volume. These are the analogues of the infinite volume estimates introduced in [dRJLS96] and proved in [dRJLS96, GK06]. We denote by $\Sigma_{\mathrm{CL}}$ the region of complete localization for the random operator $H_{\omega}$. It is defined as the set of energies $E \in \mathcal{I}$ where we have all the conclusions of the bootstrap multiscale analysis of [GK01] or the fractional moment method of [AM93, Aiz94]. These conclusions turn out to be equivalent properties describing the localized regime [GK04, GK06]. In Theorem 6.1, we provided new equivalent characterizations of the region of complete localization, involving this time finite volume operators, rather than the infinite volume one. We prove

Theorem 6.1. Let $I \subset \Sigma$ be a compact interval and assume that Wegner's estimate (W) holds in $I$. For $L$ given, consider $\Lambda=\Lambda_{L}(0)$ a cube of side length $L$ centered at 0 , and denote by $\varphi_{\omega, \Lambda, j}$, $j=1, \cdots, \operatorname{tr} \mathbf{1}_{I}\left(H_{\omega}(\Lambda)\right)$, the normalized eigenvectors of $H_{\omega}(\Lambda)$ with corresponding eigenvalue in $I$. The following are equivalent

(1) $I \subset \Sigma_{\mathrm{CL}}$ 
(2) For all $E \in I$, there exists $\theta>3 d-1$,

$$
\limsup _{L \rightarrow \infty} \mathbb{P}\left\{\forall x, y \in \Lambda,|x-y| \geq \frac{L}{2},\left\|\chi_{x}\left(H_{\omega}(\Lambda)-E\right)^{-1} \chi_{y}\right\| \leq L^{-\theta}\right\}=1 .
$$

(3) For all $\xi \in(0,1)$,

$$
\sup _{y \in \Lambda} \mathbb{E}\left\{\sum_{x \in \Lambda} \mathrm{e}^{|x-y|^{\xi}} \sup _{j}\left\|\varphi_{\omega, \Lambda, j}\right\|_{x}\left\|\varphi_{\omega, \Lambda, j}\right\|_{y}\right\}<\infty .
$$

(4) There exists $\xi \in(0,1)$,

$$
\sup _{y \in \Lambda} \mathbb{E}\left\{\sum_{x \in \Lambda} \mathrm{e}^{|x-y|^{\xi}} \sup _{j}\left\|\varphi_{\omega, \Lambda, j}\right\|_{x}\left\|\varphi_{\omega, \Lambda, j}\right\|_{y}\right\}<\infty .
$$

(5) For all $\xi \in(0,1)$,

$$
\sup _{y \in \Lambda} \mathbb{E}\left\{\sum_{x \in \Lambda} \mathrm{e}^{|x-y|^{\xi}} \sup _{\substack{\operatorname{supp} f \subset I \\|f| \leq 1}}\left\|\chi_{x} f\left(H_{\omega}(\Lambda)\right) \chi_{y}\right\|_{2}\right\}<\infty .
$$

(6) There exists $\xi \in(0,1)$,

$$
\sup _{y \in \Lambda} \mathbb{E}\left\{\sum_{x \in \Lambda} \mathrm{e}^{|x-y|^{\xi}} \sup _{\substack{\operatorname{supp} f \subset I \\|f| \leq 1}}\left\|\chi_{x} f\left(H_{\omega}(\Lambda)\right) \chi_{y}\right\|_{2}\right\}<\infty .
$$

(7) There exists $\xi \in(0,1)$,

$$
\sup _{y \in \Lambda} \sup _{\substack{\operatorname{supp} f \subset I \\|f|<1}} \mathbb{E}\left\{\sum_{x \in \Lambda} \mathrm{e}^{|x-y|^{\xi}}\left\|\chi_{x} f\left(H_{\omega}(\Lambda)\right) \chi_{y}\right\|_{2}\right\}<\infty .
$$

(8) (SUDEC for finite volume with polynomial probability) For all $p>d$, there is $q=q_{p, d}$ so that for all $\xi \in(0,1)$, for any $L$ large enough, the following holds with probability at least $1-L^{-p}$ : for any eigenvector $\varphi_{\omega, \Lambda, j}$ of $H_{\omega, \Lambda}$, with energy in $I$, for any $(x, y) \in \Lambda^{2}$, one has

$$
\left\|\varphi_{\omega, \Lambda, j}\right\|_{x}\left\|\varphi_{\omega, \Lambda, j}\right\|_{y} \leq L^{q} \mathrm{e}^{-|x-y|^{\xi}}
$$

(9) (SULE for finite volume with polynomial probability) For all $p>d$, there is $q=q_{p, d}$ so that $\xi \in(0,1)$, for any $L$ large enough, the following holds with probability at least $1-L^{-p}:$ for any eigenvector $\varphi_{\omega, \Lambda, j}$ of $H_{\omega, \Lambda}$, with energy in $I$, there is a center of localization $x_{\omega, \Lambda, j} \in \Lambda$, so that for any $x \in \Lambda$, one has

$$
\left\|\varphi_{\omega, \Lambda, j}\right\|_{x} \leq L^{q} \mathrm{e}^{-\left|x-x_{\omega, \Lambda, j}\right|^{\xi}}
$$

(10) (SUDEC for finite volume with subexponential probability) For all $\nu, \xi \in(0,1), \nu<\xi$, for any $L$ large enough, the following holds with probability at least $1-\mathrm{e}^{-L^{\nu}}$ : for any eigenvector $\varphi_{\omega, \Lambda, j}$ of $H_{\omega, \Lambda}$, with energy in $I$, for any $(x, y) \in \Lambda^{2}$, one has

$$
\left\|\varphi_{\omega, \Lambda, j}\right\|_{x}\left\|\varphi_{\omega, \Lambda, j}\right\|_{y} \leq \mathrm{e}^{2 L^{\nu}} \mathrm{e}^{-|x-y|^{\xi}} .
$$

(11) (SULE for finite volume with subexponential probability) For all $\nu, \xi \in(0,1), \nu<\xi$, for any $L$ large enough, the following holds with probability at least $1-\mathrm{e}^{-L^{\nu}}$ : for any eigenvector $\varphi_{\omega, \Lambda, j}$ of $H_{\omega, \Lambda}$, with energy in $I$, there is a center of localization $x_{\omega, \Lambda, j} \in \Lambda$, so that for any $x \in \Lambda$, one has

$$
\left\|\varphi_{\omega, \Lambda, j}\right\|_{x} \leq \mathrm{e}^{2 L^{\nu}} \mathrm{e}^{-\left|x-x_{\omega, \Lambda, j}\right|^{\xi}}
$$


Moreover one can pick $q=p+d$ in (8) and $q=p+\frac{3}{2} d$ in (9).

Remark 6.1. Theorem 6.1 provides a finite volume analog of [GK06, Theorem 1 and Corollary 3]. If generalized eigenfunctions are needed in the infinite volume case, the normalized eigenfunctions are good enough in the finite volume one for the spectrum intersected with the interval $I$ is discrete. For discrete models, using e.g. the finite volume fractional moment criteria of [ASFH01], one can derive bounds of the same type as (6.8) where $\xi=1$. This has been done in [K111].

Proof of Theorem 6.1. We start by describing precisely $\Sigma_{\mathrm{CL}}$, which is the set of energies where the conclusion of the bootstrap multiscale analysis of [GK01] is valid: $\Sigma_{\mathrm{CL}}$ for the random operator $H_{\omega}$ is defined as the set of $E \in I$ for which there exists some open interval $I(E) \subset I$, with $E \in I(E)$, such that, given any $\zeta \in(0,1)$ and $\alpha \in\left(1, \zeta^{-1}\right)$, there exists a length scale $L_{0} \in 6 \mathbb{N}$ and a mass $m>0$, so that if we set $L_{k+1}=\left[L_{k}^{\alpha}\right]_{6 \mathbb{N}}$ for $k=0,1, \ldots$, we have

$$
\mathbb{P}\left\{R\left(m, L_{k}, I(E), x, y\right)\right\} \geq 1-\mathrm{e}^{-L_{k}^{\zeta}}
$$

for all $k=0,1, \ldots$, and $x, y \in \mathbb{Z}^{d}$ with $|x-y|>L_{k}+R_{0}$, where

$$
R(m, L, I, x, y)=\left\{\begin{array}{r}
\text { for every } E^{\prime} \in I, \text { either } \Lambda_{L}(x) \\
\quad \text { or } \Lambda_{L}(y) \text { is }\left(\omega, m, E^{\prime}\right) \text {-regular }
\end{array}\right\} .
$$

Here $[K]_{6 \mathbb{N}}=\max \{L \in 6 \mathbb{N} ; L \leq K\}$; we work with scales in $6 \mathbb{N}$ for convenience; $R_{0}>0$ is given in Assumption (IAD).

Given $E \in \mathbb{R}, x \in \mathbb{Z}^{d}$ and $L \in 6 \mathbb{N}$, we say that the box $\Lambda_{L}(x)$ is $(\omega, m, E)$-regular for a given $m>0$ if $E \notin \sigma\left(H_{\omega}\left(\Lambda_{L}(x)\right)\right.$ and

$$
\left\|\Gamma_{x, L} R_{\omega, x, L}(E) \chi_{x, \frac{L}{3}}\right\| \leq \mathrm{e}^{-m L}
$$

where $R_{\omega, x, L}(E)=\left(H_{\omega}\left(\Lambda_{L}(x)\right)-E\right)^{-1}$ and $\Gamma_{x, L}$ denotes the charateristic function of the "belt" $\bar{\Lambda}_{L-1}(x) \backslash \Lambda_{L-3}(x)$ when $\mathcal{H}=\mathrm{L}^{2}\left(\mathbb{R}^{d}, \mathrm{~d} x\right)$ (the arguments can be easily modified for $\mathcal{H}=\ell^{2}\left(\mathbb{Z}^{d}\right)$ ). The interval $I$ in Theorem 6.1 being compact, we can extract from $\cup_{E \in I} I(E) \supset I$ a finite number of intervals $I(E)$ that cover $I$. Thus, with no loss, we may assume that (6.11) is valid for the interval $I$ itself.

We turn to the proof of Theorem 6.1 per se. That $(1) \Longleftrightarrow(2)$ is due to [GK04]. Note that if (6.1) holds, then

$$
\limsup _{L \rightarrow \infty} \mathbb{P}\left\{\left\|\Gamma_{0, L} R_{\omega, 0, L}(E) \chi_{0, \frac{L}{3}}\right\| \leq L^{-\theta-2 d+1}\right\}=1
$$

Assume (2), that is (6.11) holds for the interval $I$. We show that (3) holds. A standard computation (see [GK04, (EDI)], [Kir08, Eq.(8.10)]) shows that if $\Lambda_{L}(x)$ is $(\omega, m, E)$-regular, then, if $H_{\omega}\left(\Lambda_{L}(x)\right) \varphi_{\omega, \Lambda, j}=\tilde{E} \varphi_{\omega, \Lambda, j}$ and $\left\|\varphi_{\omega, \Lambda, j}\right\|=1$, then

$$
\left\|\varphi_{\omega, \Lambda, j}\right\|_{x} \lesssim L^{d-1}\left\|\Gamma_{x, L} R_{\omega, x, L}(\tilde{E}) \chi_{x, \frac{L}{3}}\right\| \leq \mathrm{e}^{-\frac{m}{2} L}
$$

whenever $L$ is large enough, depending only on $d, m$.

It follows that for any $(x, y) \in \Lambda^{2}$, if $k$ is so that $L_{k} \leq|x-y|<L_{k+1}$, then for any $\omega \in$ $R(m, L, I, x, y)$, for any normalized $\varphi_{\omega, \Lambda, j}$,

$$
\left\|\varphi_{\omega, \Lambda, j}\right\|_{x}\left\|\varphi_{\omega, \Lambda, j}\right\|_{y} \leq \mathrm{e}^{-\frac{m}{2} L_{k}} \leq \mathrm{e}^{-\frac{m}{2}|x-y|^{\frac{1}{\alpha}}} .
$$

As a consequence,

$$
\mathbb{E}\left\{\sup _{j}\left\|\varphi_{\omega, \Lambda, j}\right\|_{x}\left\|\varphi_{\omega, \Lambda, j}\right\|_{y}\right\} \leq \mathrm{e}^{-\frac{m}{2}|x-y|^{\frac{1}{\alpha}}}+\mathrm{e}^{-|x-y|^{\frac{\zeta}{\alpha}}} \leq 2 \mathrm{e}^{-|x-y|^{\frac{\zeta}{\alpha}}},
$$

for $|x-y|$ large enough. Thus, (6.2) follows for any $\xi<\zeta \alpha^{-1}$. Since $\zeta<1<\alpha$ can be chosen arbitrarily close to $1,(3)$ follows. 
(3) clearly implies (4).

To see that (3) implies (5), it is enough to decompose the operator $f\left(H_{\omega}(\Lambda)\right)$ over the eigenvectors $\left(\varphi_{\omega, \Lambda, j}\right)_{j}$ and note that $\left\|\chi_{x} \Pi_{\varphi_{\omega, \Lambda, j}} \chi_{y}\right\|_{2}=\left\|\varphi_{\omega, \Lambda, j}\right\|_{x}\left\|\varphi_{\omega, \Lambda, j}\right\|_{y}$, when $\Pi_{\varphi_{\omega, \Lambda, j}}$ is the orthogonal projection on $\varphi_{\omega, \Lambda, j}$.

That $(5) \Longrightarrow(6) \Longrightarrow(7)$ is immediate.

Assume (3). We prove (8). We have

$$
\begin{aligned}
& \mathbb{P}\left(\exists(x, y) \in \Lambda^{2}, \mathrm{e}^{|x-y|^{\xi}} \sup _{j}\left\|\varphi_{\omega, \Lambda, j}\right\|_{x}\left\|\varphi_{\omega, \Lambda, j}\right\|_{y} \geq L^{p+d}\right) \\
& \leq L^{d} \sup _{y \in \Lambda} \sum_{x \in \Lambda} \mathbb{P}\left(\mathrm{e}^{|x-y|^{\xi}} \sup _{j}\left\|\varphi_{\omega, \Lambda, j}\right\|_{x}\left\|\varphi_{\omega, \Lambda, j}\right\|_{y} \geq L^{p+d}\right) \\
& \leq L^{-p} \sup _{y \in \Lambda} \sum_{x \in \Lambda} \mathbb{E}\left(\mathrm{e}^{|x-y|^{\xi}} \sup _{j}\left\|\varphi_{\omega, \Lambda, j}\right\|_{x}\left\|\varphi_{\omega, \Lambda, j}\right\|_{y}\right) \\
& \quad \lesssim L^{-p} .
\end{aligned}
$$

In other terms, with probability at least $1-L^{-p}$, we have $\left\|\varphi_{\omega, \Lambda, j}\right\| x\left\|\varphi_{\omega, \Lambda, j}\right\|_{y} \| \leq L^{p+d} \mathrm{e}^{-|x-y|^{\xi}}$, for any $j$ and $(x, y) \in \Lambda^{2}$, and (8) holds.

We show that (8) and (9) are equivalent. Assume (8) and let $x_{\omega, \Lambda, j}$ be a center of localization for $\varphi_{\omega, \Lambda, j}$. Since $\left\|\varphi_{\omega, \Lambda, j}\right\|=1$, note that $\left\|\varphi_{\omega, \Lambda, j}\right\|_{x} \gtrsim L^{-d / 2}$. We write (6.7) with $y=x_{\omega, \Lambda, j}$ and (6.8) follows from the last observation with a constant $q^{\prime}=q+\frac{d}{2}$. Conversely, if (9) holds, then it follows from (6.8) that

$$
\left\|\varphi_{\omega, \Lambda, j}\right\|_{x}\left\|\varphi_{\omega, \Lambda, j}\right\|_{y} \leq L^{2 q} \mathrm{e}^{-\left|x-x_{\omega, \Lambda, j}\right|^{\xi}} \mathrm{e}^{-\left|y-x_{\omega, \Lambda, j}\right|^{\xi}} \leq L^{2 q} \mathrm{e}^{-|x-y|^{\xi}} .
$$

We show that (8) $\Longrightarrow(2)$. Assume (8). For any given $E \in I$, thanks to (W), we may assume that $\mathrm{d}\left(E, \sigma\left(H_{\omega}(\Lambda)\right)\right) \geq L^{-p}$, with probability at least $1-L^{-p+d}$. As a consequence, for any $L$ large enough, for any $(x, y) \in \Lambda^{2},|x-y| \geq L / 2$,

$$
\begin{aligned}
\left\|\chi_{x}\left(H_{\omega}(\Lambda)-E\right)^{-1} \chi_{y}\right\| & \leq L^{p} \sum_{j}\left\|\varphi_{\omega, \Lambda, j}\right\|_{x}\left\|\varphi_{\omega, \Lambda, j}\right\|_{y} \\
& \leq L^{p+q} \mathrm{e}^{-L^{\xi}} .
\end{aligned}
$$

The estimate (2) follows, regardless of the values of $p, q, \xi$.

The proofs related to (10) and (11) are similar.

It remains to show (7) $\Longrightarrow(2)$. Assume (7) and pick $E \in I$. Set $\delta=L^{-p}$. By (W), with probability at least $1-L^{-p}$, we have

$$
\left(H_{\omega}(\Lambda)-E\right)^{-1}=\left(H_{\omega}(\Lambda)-E\right)^{-1} \mathbf{1}_{\Sigma \backslash[E-\delta, E+\delta]}\left(H_{\omega}(\Lambda)\right) .
$$


Define the function $f_{E, \delta}(\lambda)=\delta(\lambda-E)^{-1} \mathbf{1}_{\Sigma \backslash[E-\delta, E+\delta]}(\lambda)$. Note that $\left|f_{E, \delta}\right| \leq 1$. It follows that

$$
\begin{aligned}
& \mathbb{P}\left(\exists(x, y) \in \Lambda^{2},|x-y| \geq \frac{L}{2},\left\|\chi_{x}\left(H_{\omega}(\Lambda)-E\right)^{-1} \chi_{y}\right\| \geq L^{-\theta}\right) \\
& \leq L^{-p}+\sum_{\substack{(x, y) \in \Lambda^{2} \\
|x-y| \geq L / 2}} \mathbb{P}\left(\left\|\chi_{x} f_{E, \delta}\left(H_{\omega}(\Lambda)\right) \chi_{y}\right\| \geq L^{-\theta-p}\right) \\
& \quad \leq L^{-p}+L^{-p} \sup _{y \in \Lambda} \sum_{\substack{x \in \Lambda \\
|x-y| \geq L / 2}} L^{\theta+2 p+d} \mathbb{E}\left(\left\|\chi_{x} f_{E, \delta}\left(H_{\omega}(\Lambda)\right) \chi_{y}\right\|\right) \\
& \quad \leq L^{-p}+L^{-p} \sup _{y \in \Lambda} \sum_{x \in \Lambda}(2|x-y|)^{\theta+2 p+d} \mathbb{E}\left(\left\|\chi_{x} f_{E, \delta}\left(H_{\omega}(\Lambda)\right) \chi_{y}\right\|_{2}\right) \lesssim L^{-p} .
\end{aligned}
$$

The last observation stated in the theorem follows from the proof above.

\section{REFERENCES}

$\left[\mathrm{AEN}^{+}\right.$06] Michael Aizenman, Alexander Elgart, Serguei Naboko, Jeffrey H. Schenker, and Gunter Stolz. Moment analysis for localization in random Schrödinger operators. Invent. Math., 163(2):343-413, 2006.

[Aiz94] Michael Aizenman. Localization at weak disorder: some elementary bounds. Rev. Math. Phys., 6(5A):1163-1182, 1994. Special issue dedicated to Elliott H. Lieb.

[AM93] Michael Aizenman and Stanislav Molchanov. Localization at large disorder and at extreme energies: an elementary derivation. Comm. Math. Phys., 157(2):245-278, 1993.

[ASFH01] Michael Aizenman, Jeffrey H. Schenker, Roland M. Friedrich, and Dirk Hundertmark. Finite-volume fractional-moment criteria for Anderson localization. Comm. Math. Phys., 224(1):219-253, 2001. Dedicated to Joel L. Lebowitz.

[BCKP88] Anton Bovier, Massimo Campanino, Abel Klein, and J. Fernando Perez. Smoothness of the density of states in the Anderson model at high disorder. Comm. Math. Phys., 114(3):439-461, 1988.

[BHS07] Jean V. Bellissard, Peter D. Hislop, and Günter Stolz. Correlation estimates in the Anderson model. J. Stat. Phys., 129(4):649-662, 2007.

[CGK09] Jean-Michel Combes, François Germinet, and Abel Klein. Generalized eigenvalue-counting estimates for the Anderson model. J. Stat. Phys., 135(2):201-216, 2009.

[dRJLS96] Rafael del Rio, Svetlana Jitomirskaya, Yoram Last, and Barry Simon. Operators with singular continuous spectrum. IV. Hausdorff dimensions, rank one perturbations, and localization. J. Anal. Math., 69:153200, 1996.

[Dur96] Richard Durrett. Probability: theory and examples. Duxbury Press, Belmont, CA, second edition, 1996.

[GDB98] François Germinet and Stephan De Bièvre. Dynamical localization for discrete and continuous random Schrödinger operators. Comm. Math. Phys., 194(2):323-341, 1998.

[GHK07] Fatma Ghribi, Peter D. Hislop, and Frédéric Klopp. Localization for Schrödinger operators with random vector potentials. In Adventures in mathematical physics, volume 447 of Contemp. Math., pages 123-138. Amer. Math. Soc., Providence, RI, 2007.

[GKl11] François Germinet and Frédéric Klopp. Spectral statistics for the discrete Anderson model in the localized regime. In Spectra of random operators and related topics, RIMS Kôkyûroku Bessatsu, B27, pages 11-24. Res. Inst. Math. Sci. (RIMS), Kyoto, 2011.

[GKl12] François Germinet and Frédéric Klopp. Enhanced Wegner and Minami estimates and eigenvalue statistics of random Anderson models at spectral edges To appear in Annales Henri Poincaré. ArXiv http://arxiv.org/abs/1111.1505.

[GK01] François Germinet and Abel Klein. Bootstrap multiscale analysis and localization in random media. Comm. Math. Phys., 222(2):415-448, 2001.

[GK04] François Germinet and Abel Klein. A characterization of the Anderson metal-insulator transport transition. Duke Math. J., 124(2):309-350, 2004.

[GK06] François Germinet and Abel Klein. New characterizations of the region of complete localization for random Schrödinger operators. J. Stat. Phys., 122(1):73-94, 2006.

[GV07] Gian Michele Graf and Alessio Vaghi. A remark on the estimate of a determinant by Minami. Lett. Math. Phys., 79(1):17-22, 2007. 
[His08] Peter D. Hislop. Lectures on random Schrödinger operators. In Fourth Summer School in Analysis and Mathematical Physics, volume 476 of Contemp. Math., pages 41-131. Amer. Math. Soc., Providence, RI, 2008.

[HK02] Peter D. Hislop and Frédéric Klopp. The integrated density of states for some random operators with nonsign definite potentials. J. Funct. Anal., 195(1):12-47, 2002.

[Jan98] Martin Janssen. Statistics and scaling in disordered mesoscopic electron systems. Physics Reports, 295(12):1-91, March 1998.

[Kir08] Werner Kirsch. An invitation to random Schrödinger operators. In Random Schrödinger operators, volume 25 of Panor. Synthèses, pages 1-119. Soc. Math. France, Paris, 2008. With an appendix by Frédéric Klopp.

[KM07] Werner Kirsch and Bernd Metzger. The integrated density of states for random Schrödinger operators. In Spectral theory and mathematical physics: a Festschrift in honor of Barry Simon's 60th birthday, volume 76 of Proc. Sympos. Pure Math., pages 649-696. Amer. Math. Soc., Providence, RI, 2007.

[KN07] Rowan Killip and Fumihiko Nakano. Eigenfunction statistics in the localized Anderson model. Ann. Henri Poincaré, 8(1):27-36, 2007.

[K195] Frédéric Klopp. Localization for some continuous random Schrödinger operators. Comm. Math. Phys., 167(3):553-569, 1995.

[Kl11] Frédéric Klopp. Decorrelation estimates for the discrete Anderson model. Comm. Math. Phys., 303(1):233-260, 2011.

[K112] Frédéric Klopp. Inverse tunneling estimates and applications to the study of spectral statistics of random operators on the real line. To appear in Crelle's Journal. ArXiv: http://fr.arxiv.org/abs/1101.0900.

[Kri08] Evgenij Kritchevski. Poisson statistics of eigenvalues in the hierarchical Anderson model. Ann. Henri Poincaré, 9(4):685-709, 2008.

[LR85] Patrick A. Lee and T. V. Ramakrishnan. Disordered electronic systems. Rev. Mod. Phys., 57(2):287-337, Apr 1985.

[Min96] Nariyuki Minami. Local fluctuation of the spectrum of a multidimensional Anderson tight binding model. Comm. Math. Phys., 177(3):709-725, 1996.

[Min07] Nariyuki Minami. Theory of point processes and some basic notions in energy level statistics. In Probability and mathematical physics, volume 42 of CRM Proc. Lecture Notes, pages 353-398. Amer. Math. Soc., Providence, RI, 2007.

[Min08] Nariyuki Minami. Energy level statistics for random operators. In Friedrich Götze, Werner Kirsch, Frédéric Klopp, and Thomas Kriecherbauer, editors, Disordered systems: random Schrödinger operators and random matrices, volume 5 of Oberwolfach Reports, pages 842-844, 2008.

[Mir00] Alexander Mirlin. Statistics of energy levels and eigenfunctions in disordered systems. Physics Reports, 326(5-6):259-382, March 2000.

[Mol82] Stanislav A. Molchanov. The local structure of the spectrum of a random one-dimensional Schrödinger operator. Trudy Sem. Petrovsk., (8):195-210, 1982.

[PF92] Leonid Pastur and Alexander Figotin. Spectra of random and almost-periodic operators, volume 297 of Grundlehren der Mathematischen Wissenschaften [Fundamental Principles of Mathematical Sciences]. Springer-Verlag, Berlin, 1992.

[PS98] George Pólya and Gabor Szegő. Problems and theorems in analysis. I. Classics in Mathematics. SpringerVerlag, Berlin, 1998. Series, integral calculus, theory of functions, Translated from the German by Dorothee Aeppli, Reprint of the 1978 English translation.

[Pyk65] Ronald Pyke. Spacings. (With discussion.). J. Roy. Statist. Soc. Ser. B, 27:395-449, 1965.

[RS78] Michael Reed and Barry Simon. Methods of modern mathematical physics. IV. Analysis of operators. Academic Press [Harcourt Brace Jovanovich Publishers], New York, 1978.

[RS79] Michael Reed and Barry Simon. Methods of Modern Mathematical Physics, Vol II: Fourier Analysis, Self-Adjointness. Academic Press, New-York, 1979.

[Sim05] Barry Simon. Trace ideals and their applications, volume 120 of Mathematical Surveys and Monographs. American Mathematical Society, Providence, RI, second edition, 2005.

[Sto01] Peter Stollmann. Caught by disorder, volume 20 of Progress in Mathematical Physics. Birkhäuser Boston Inc., Boston, MA, 2001. Bound states in random media.

[Tho74] David Thouless. Electrons in disordered systems and the theory of localization. Physical Reports, 13:93$142,1974$.

[Ves08] Ivan Veselić. Existence and regularity properties of the integrated density of states of random Schrödinger operators, volume 1917 of Lecture Notes in Mathematics. Springer-Verlag, Berlin, 2008.

[Wan01] Wei-Min Wang. Localization and universality of Poisson statistics for the multidimensional Anderson model at weak disorder. Invent. Math., 146(2):365-398, 2001. 
[Wei55] Lionel Weiss. The stochastic convergence of a function of sample successive differences. Ann. Math. Statist., 26:532-536, 1955.

(François Germinet) Université de Cergy-Pontoise, CNRS UMR 8088, IUF, Département de mathématiques, F-95000 Cergy-Pontoise, France

E-mail address: francois.germinet@u-cergy.fr

(Frédéric Klopp) IMJ, UMR CNRS 7586 Université Pierre et Marie Curie Case 186, 4 place Jussieu F-75252 PARIS CEDEX 05 France

E-mail address: klopp@math.jussieu.fr 